SURFACE ENGINEERING OF $\bar{\propto}$

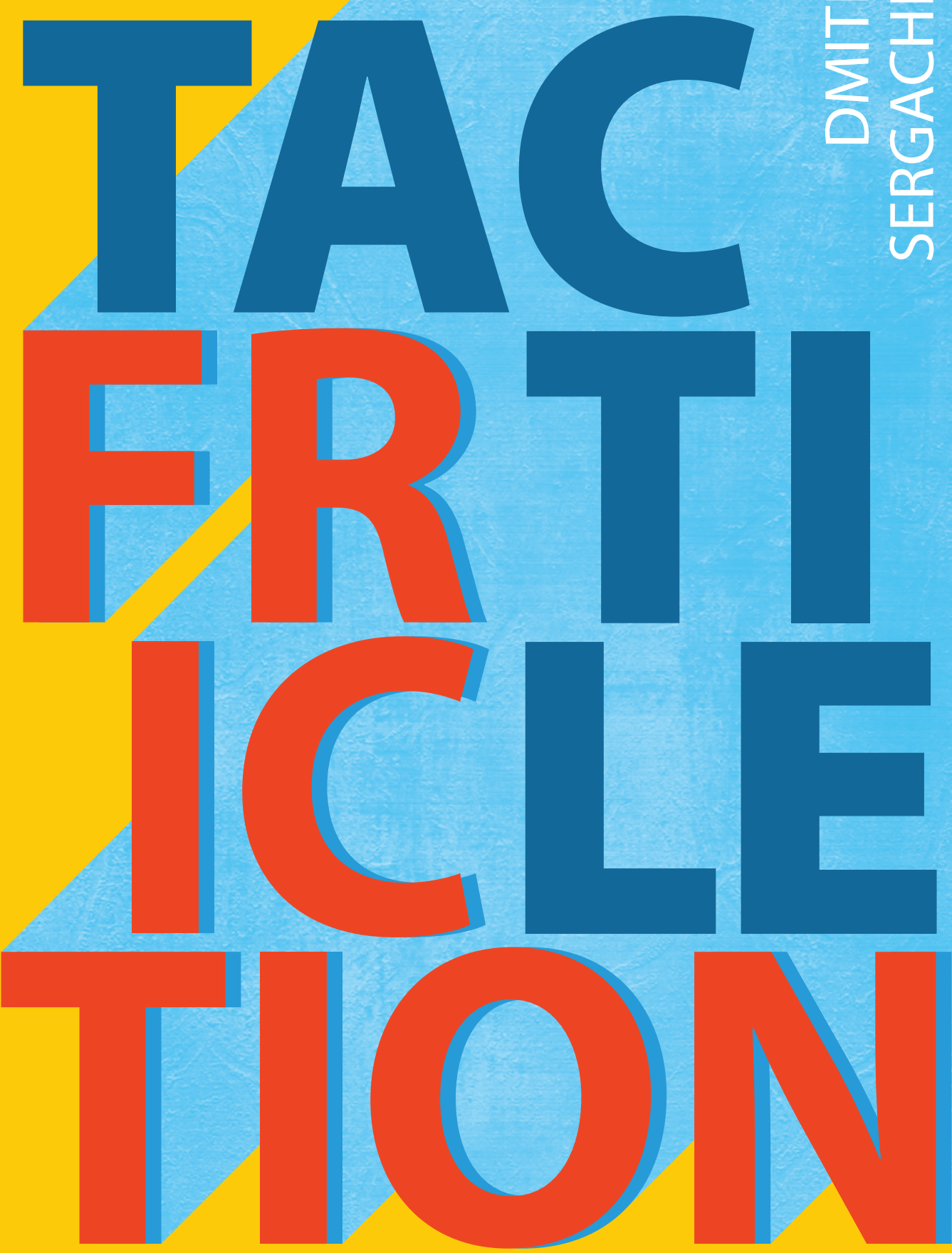




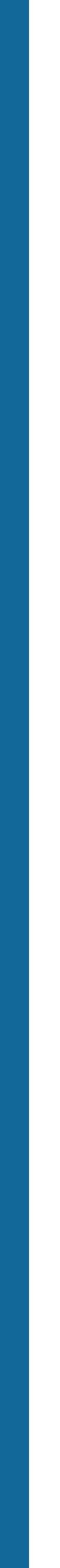


SURFACE ENGINEERING OF

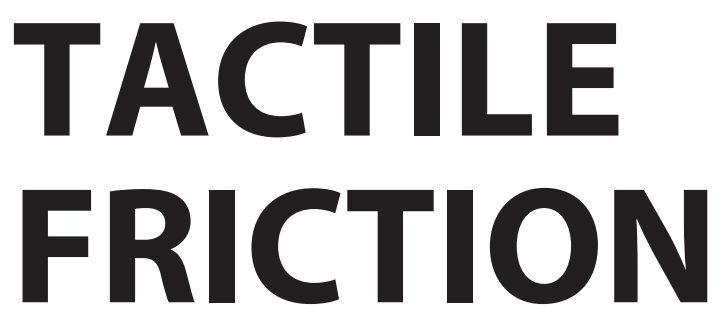

DMITRII SERGACHEV 
COMPOSITION OF THE GRADUATION COMMITTEE:

Chairman and secretary:

Prof. dr. ir. H.F.J.M. Koopman

University of Twente

Promotor:

Prof. dr. ir. E. van der Heide

University of Twente

Co-promotor:

Dr. D.T.A. Matthews

University of Twente

Members:

Prof. dr. ir. A.H. van den Boogaard

University of Twente

Prof. R. Lewis

University of Sheffield

Prof. dr. ir. G.R.B.E. Römer

University of Twente

Dr. L. Skedung

RISE Research Institutes of Sweden

Prof. dr. ir. A.A. Zadpoor

Delft University of Technology

This work was financially supported by INTERREG V-A Deutschland - Nederland program MOVERO (www.deutschland-nederland.eu) under the project number 142091.

\section{COLOPHON}

Cover design: Ilse Schrauwers, isontwerp.nl

Printed by: Drukkerij Wihabo, Geffen

ISBN: 978-90-365-5178-6

DOI: $10.3990 / 1.9789036551786$

Copyright (C2021, Dmitrii Aleksandrovich Sergachev, The Netherlands. 


\title{
SURFACE ENGINEERING OF \\ TACTILE FRICTION
}

\section{DISSERTATION}

\author{
to obtain \\ the degree of doctor at the University of Twente, \\ on the authority of the rector magnificus, \\ Prof. dr. ir. A. Veldkamp, \\ on account of the decision of the Doctorate Board, \\ to be publicly defended \\ on Friday the $2^{\text {nd }}$ of July 2021 at 16:45 hours
}

by

\section{DMITRII ALEKSANDROVICH SERGACHEV}

Born on the $8^{\text {th }}$ of May 1990

in Mytishchi, Russia 
This dissertation has been approved by the promotor

Prof. dr. ir. E. van der Heide

and co-promotor

Dr. D.T.A. Matthews 
To people met by chance, whose names I don't always know, but who shared nuggets of information and opened new opportunities. 



\section{SUMMARY}

In everyday life, people interact with numerous products through touch. A massive amount of information is generated through such tactile contact with a product surface. Surfaces thus influence the perception of the product physical properties, grip performance and forces applied during object handling and manipulation. Tactile perception can be modified by surface engineering. In this thesis that is achieved by producing deterministic surface topographies with designed frictional performance. This approach can improve a vast variety of products from sport and medical equipment to consumer products and packaging.

The main aim of the current work is to control and enhance tactile perception through modification of the frictional behaviour by surface topography design. Following a systematic approach, an asperity and texture design was selected for this research. A contact model was developed to predict finger pad contact area on the micro- and macroscales. The model was applied to understand the role of component parts in a tactile tribological system - namely to estimate skin elastic modulus, characterise skin deformation and determine the role of varying surface texture dimensions. The influence of texture parameters and individual finger properties were evaluated through experimental and numerical studies. Texture dimensions were related to tactile perception and friction effects reported in literature, which resulted in the foundation of a design map for surface design in tactile contacts.

The contact model is developed combining numerical and analytical methods to calculate contact area of the finger pad in contact with deterministic micro-textured surfaces. The boundary element method is applied to calculate skin contact area on the micro-scale. It allows to predict the contact transition effect expressed in the change from the asperity supported state to the full contact with the surface. The analytical method is used to obtain the simulation parameters from the macroscale. The model was applied to estimate skin elasticity by observation of the contact transition through friction measurements. The 
effective elastic modulus of the skin, applicable on the microscale, was estimated empirically to be in the range between $0.2 \mathrm{MPa}$ and $0.5 \mathrm{MPa}$.

A significant influence of the skin contact state on tactile friction was shown experimentally. Deterministic surfaces, which remained in asperity contact, showed a considerable reduction of friction coefficient. A bidirectional frictional behaviour was achieved with ellipsoidal texture design and was correlated to the feature geometry and material properties. The results showed the feasibility of the asymmetric texture designs for tactile friction, which are based on the change in the contact area and not the deformation of the skin. The friction measurements performed with a group of volunteers, with the aim of normalisation, show that the reference sample can be used to normalise and compare values between individuals. Furthermore, the participants showed a comparable range for the skin micro-deformation, suggesting that the same textured surfaces have a similar functional performance for a group of people.

A foundation for a texture design map is developed, towards establishing a connection between the texture dimensions and effects attributed to tactile friction. The design map can be used as a reference for the geometrical boundaries in surface texture design with the aim to control and predict frictional behaviour and to enhance tactile perception. 


\section{SAMENVATTING}

\section{Het ontwerpen en construeren van oppervlakken voor tactiele wrijving}

In het dagelijks leven vinden talrijke interacties met producten plaats door aanraking. Het tactiele contact met de productoppervlakken genereert een enorme hoeveelheid informatie, die de perceptie van de fysieke eigenschappen van het product, gripprestaties en uitgeoefende krachten beïnvloeden tijdens hanteren en manipuleren van de objecten. Tactiele waarneming kan worden veranderd door het ontwerpen en construeren van oppervlakken. In dit proefschrift dat wordt bereikt door het produceren van deterministische oppervlaktetopografieën met ontworpen wrijvingsgedrag. Deze aanpak kan een grote verscheidenheid aan producten verbeteren, van sport- en medische apparatuur tot consumentenproducten en verpakkingen.

Het belangrijkste doel van dit proefschrift is het beheersen en verbeteren van de tactiele waarneming van productoppervlakken door het aanpassen van het wrijvingsgedrag met het ontwerpen en construeren van de oppervlaktetopografie. Als onderdeel van een systematische aanpak werd voor dit onderzoek gekozen voor een ruwheidstop- en textuurontwerp. Er is een contactmodel ontwikkeld om het contactoppervlak van de vingertop te voorspellen zowel op de micro- als op en macroschaal. Het contactmodel werd toegepast om de rol van componenten in een tactiel tribologisch systeem te begrijpen - namelijk om de elasticiteitsmodulus van de huid te schatten, contactdeformatie van de huid te karakteriseren en de rol van verschillende textuurafmetingen daarop te bepalen. De invloed van textuurparameters en individuele vingereigenschappen zijn geëvalueerd door middel van experimentele en numerieke studies. Textuurafmetingen zijn gerelateerd aan tactiele perceptie en wrijvingseffecten uit de literatuur, wat geresulteerd heeft in de basis van een grafiek - een design map - voor het ontwerpen van oppervlakken in tactiele contacten. 
Het contactmodel is ontwikkeld als een combinatie van numerieke en analytische methoden waarmee het contactgebied van de vingertop te berekenen is, in contact met deterministische micro-oppervlaktetexturen. De boundary element method is toegepast om het contactgebied van de huid op de microschaal te berekenen. Daarmee is het mogelijk geworden om een verandering in het contact te voorspellen, namelijk die van het contact met de toppen van de ruwheid naar een volledig contact met het oppervlak. De analysemethode is gebruikt ter bepaling van de simulatieparameters op de macroschaal. Het model werd toegepast om de elasticiteit van de huid te schatten door observatie van de contactverandering tijdens wrijvingsmetingen. De effectieve elasticiteitsmodulus van de huid op de microschaal, is empirisch bepaald en geschat op 0,2 MPa tot 0,5 MPa.

Expermenteel is aangetoond dat de contacttoestand van de huid een significante invloed heeft op tactiele wrijving. Deterministische oppervlakken, die in de contacttoestand blijven, waarbij alleen de toppen incontact zijn met de huid, vertonen een aanzienlijke vermindering in de wrijvingscoëfficiënt. Een bidirectioneel wrijvingsgedrag werd bereikt met een ellipsvormig textuurontwerp, gecorreleerd aan de objectgeometrie en materiaaleigenschappen. De resultaten laten de haalbaarheid zien van de asymmetrische textuurontwerpen voor tactiele wrijving gebaseerd op de verandering in het contactgebied in plaats van op deformatie van de huid. De wrijvingsmetingen die met een groep vrijwilligers werden uitgevoerd, met als doel normalisatie, tonen aan dat het referentieoppervlak kan worden gebruikt om waarden tussen individuen te normaliseren en te vergelijken. Bovendien toonden de deelnemers een vergelijkbaar bereik voor de microdeformaties van de huid, wat suggereert dat dezelfde getextureerde oppervlakken een vergelijkbaar functioneel gedrag opleveren voor een groep mensen.

In dit proefschrift is een basis gelegd voor een design map waarin een verband gelegd wordt tussen de textuurafmetingen en effecten gerelateerd aan tactiele wrijving. De design map kan worden gebruikt als referentie voor de geometrische grenzen in het ontwerpen van oppervlaktetexturen met als doel het beheersen en voorspellen van het wrijvingsgedrag en het verbeteren van de tactiele perceptie. 


\section{ACKNOWLEDGMENTS}

First and foremost I would like to express my sincere gratitude to my promoter Prof. dr. ir. Emile van der Heide for the given trust and freedom during the research, for his subtle guidance, patience and continuous support over the course of these years. I would like to extend my sincere thanks to my co-promoter and daily supervisor Dr. David Matthews for his invaluable corrections and suggestions during writing, for all the time, whiteboard space and coffee that he gladly provided for our discussions.

I am deeply grateful to the committee members Prof. dr. ir. Antonius van den Boogaard, Prof. Roger Lewis, Prof. dr. ir. Gert-willem Römer, Dr. Lisa Skedung and Prof. dr. ir. Amir Zadpoor for their time and professional judgement of my work.

This research would have not been possible without the financial support of INTERREG Deutschland-Nederland program MOVERO and contribution of the project partners: Schepers GmbH \& Co KG, SAUERESSIG GmbH \& Co. KG, Kamp Coating Apeldoorn BV and TAFH Münster GmbH. In particular, I would like to thank Dr. Stephan Brüning for the timely and accurate production of the designed textures, Dr. Ronny Schlegel, Renate Warmers and Roman Stoll for the conduction of hot embossing trials and subsequent analysis of the results, Marco Smarra and Nico Feima for their insightful comments and expertise, Jürgen Gröninger, Stephanie Koch and Maarten Waaijenberg for their management and project organisation.

I am grateful to all the collegues I met at the University of Twente. Special thanks to Erik de Vries, Walter Lette and Robert Jan Meijer for their help with laboratory equipment and technical questions, typically accompanied by my sudden appearance at their office door. I would like to thank Belinda and Debbie for taking care of all organizational matters and occasional distribution of free sandwiches. I am thankful to Dr. Michel Klaassen for passing to me the test rig along with the data acquisition software and to Dr. Aydar Akchurin for sharing his MATLAB code, which served as a foundation for numerical simulations. It saved a lot of time and helped to build upon your work instead of repeating it. 
Thank you Melkamu, Naveed, Can and Tanmaya for the in-depth discussions both related and unrelated to tribology. I want to thank Xavier, Faizan, Pramod, Yuxin, Pedro, Matthias, Hasib, Nadia, Marek, Luigi, Mohammad, Shivam, Matthijs, Marina, Shakil, Liangyong, Yuchen, Paloma, Shari, Dariush for all the small talks, lunch and coffee breaks, which seem especially valuable now when everybody works from home.

I would like to thank my friends Pavel, Olga, Julia, Aleksey, Ilya, Diana, Sergey, Angelina, Margarita, Alexander for the bright memories, warm hospitality and cheerfullness. I am deeply grateful to my parents for their unwavering support, to my sister Svetlana for our long discussions about life values and ethics and to my aunt Lidia for the infinite supply of handcrafted sweaters. 


\section{CONTENTS}

SUMMARY .. .1

SAMENVATTING ......................................................................................................... II

ACKNOWLEDGMENTS........................................................................................................ V

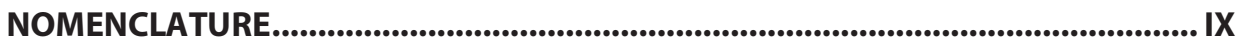

\section{PART I}

$1 \quad$ INTRODUCTION ..................................................................................................................1

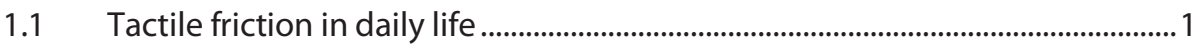

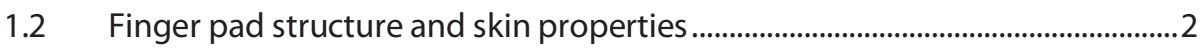

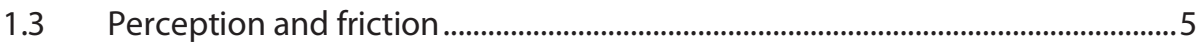

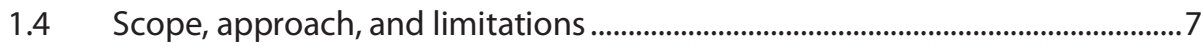

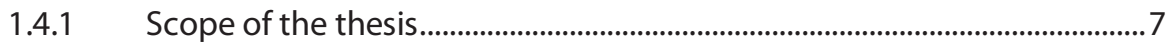

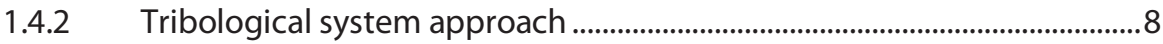

1.4.3 Systems approach and limitations.................................................................. 10

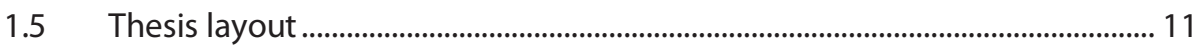

2 FRICTION AND CONTACT MECHANICS......................................................... 13

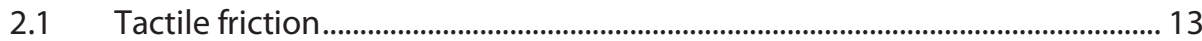

2.1.1 Two-term friction model .............................................................................. 13

2.1.2 Tactile friction against stochastic surfaces................................................ 15

2.1.3 Tactile friction against deterministic surfaces................................................ 16

2.2 Contact modelling .......................................................................................................... 18

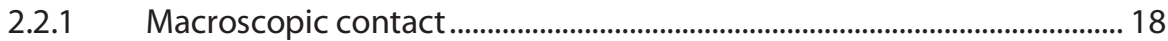

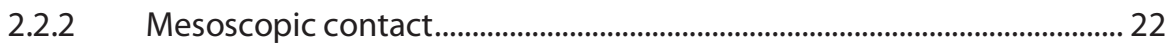

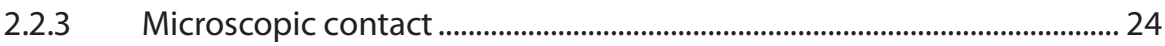

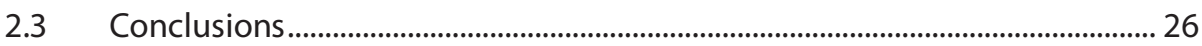


3 NUMERICAL METHODS.................................................................................. 29

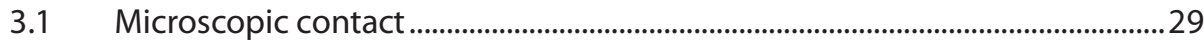

3.2 Numerical method for contact state estimation....................................................32

3.3 Experimental evaluation of the skin elastic modulus...........................................36

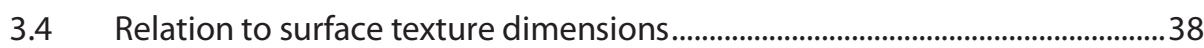

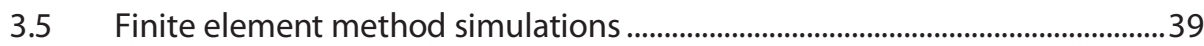

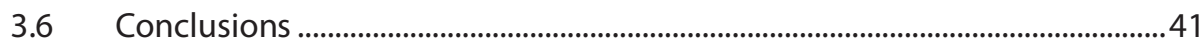

4 TACTILE FRICTION MEASUREMENTS ........................................................... 43

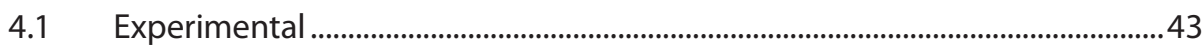

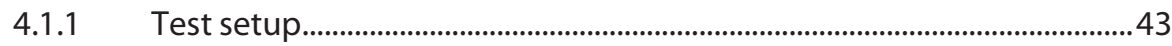

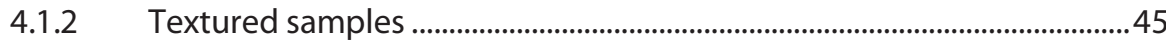

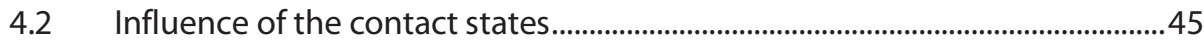

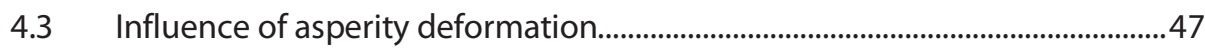

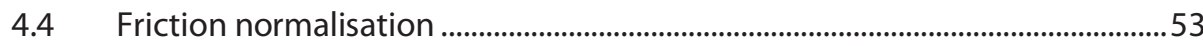

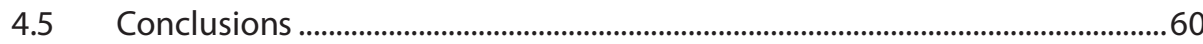

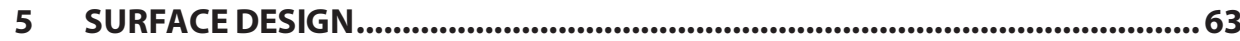

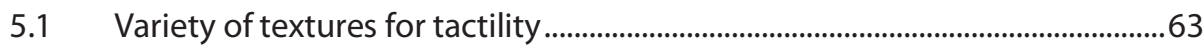

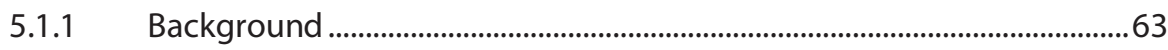

5.1.2 Overview of current texture designs for tactile surfaces...........................64

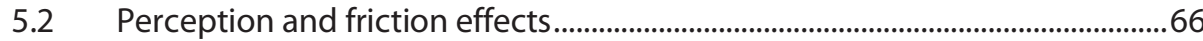

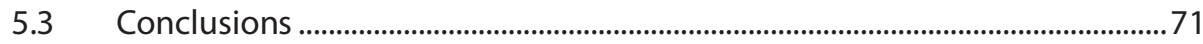

6 CONCLUSIONS AND OUTLOOK.................................................................... 73

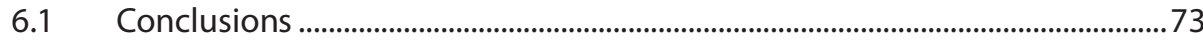

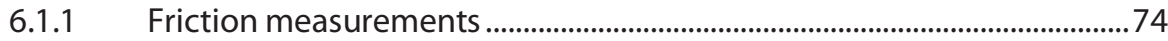

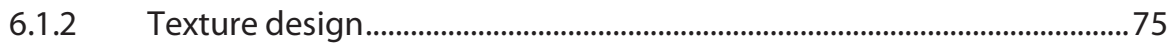

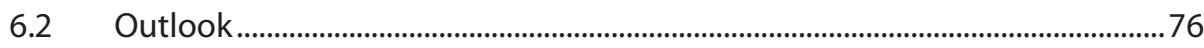

BIBILOGRAPHY ............................................................................................ 77

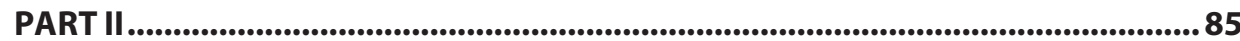




\section{NOMENCLATURE}

\section{ROMAN SYMBOLS}

\begin{tabular}{|c|c|c|}
\hline symbol & description & units \\
\hline$a_{f r}$ & $\begin{array}{l}\text { equivalent radius of a fingerprint ridge contact } \\
\text { area }\end{array}$ & $\mathrm{m}$ \\
\hline$A_{f r}$ & fingerprint ridge contact area & $\mathrm{m}^{2}$ \\
\hline$A_{r e}$ & real contact area & $\mathrm{m}^{2}$ \\
\hline$A_{r e_{r e f}}$ & $\begin{array}{l}\text { real contact area with a reference materials } \\
\text { having a nominally flat surface }\end{array}$ & $\mathrm{m}^{2}$ \\
\hline$a_{\text {trans }}$ & $\begin{array}{l}\text { equivalent radius of an apparent contact area in a } \\
\text { full contact state }\end{array}$ & $\mathrm{m}$ \\
\hline$A_{\text {trans }}$ & $\begin{array}{l}\text { fingerprint ridge contact area in a full contact } \\
\text { state }\end{array}$ & $\mathrm{m}^{2}$ \\
\hline C & constraint coefficient for secant modulus & - \\
\hline$E_{0}^{*}$ & reduced Young's modulus in macro contact & $\mathrm{Pa}$ \\
\hline$E_{1}^{*}$ & reduced Young's modulus in micro contact & $\mathrm{Pa}$ \\
\hline$E_{f p}$ & Young's modulus of a finger pad & $\mathrm{Pa}$ \\
\hline$E_{f p}{ }^{*}$ & reduced Young's modulus of the finger pad & $\mathrm{Pa}$ \\
\hline$E_{m}$ & Young's modulus of a counter surface material & $\mathrm{Pa}$ \\
\hline$E_{s}$ & $\begin{array}{l}\text { effective skin Young's modulus in micro-scale } \\
\text { contact }\end{array}$ & $\mathrm{Pa}$ \\
\hline$E_{s f p}^{*}$ & reduced secant modulus of the finger pad & $\mathrm{Pa}$ \\
\hline
\end{tabular}


F applied normal load $\quad \mathrm{N}$

$F_{\text {adh }} \quad$ interfacial adhesion friction force N

$F_{\text {crit }} \quad$ normal load at which a transition to a full contact $\mathrm{N}$ state occurs

$F_{\text {def }} \quad$ hysteresis friction force $\quad \mathrm{N}$

$F_{f} \quad$ total friction force $\quad$ N

$F_{f_{t e x}} \quad$ friction force measured for a materials having a $\quad \mathrm{N}$ textured surface

$F_{f_{\text {ref }}} \quad$ friction force measured for a reference material $\quad \mathrm{N}$ having a nominally flat surface

$F_{i} \quad$ force acting on individual asperity $\quad \mathrm{N}$

$\bar{F}_{i} \quad$ mean force acting on individual asperity $\quad \mathrm{N}$

$F_{\text {icrit }} \quad$ critical force acting on individual asperity at N which a transition to a full contact state occurs

$h_{\text {asp }}$ height of an asperity m

$k \quad$ power-law coefficient for coefficient of friction $\quad \mathrm{N}^{1-n}$

$k_{t} \quad$ dimensionless texture coefficient

$n \quad$ power-law load index for coefficient of friction

$N \quad$ number of texture asperities in contact

$\bar{p} \quad$ mean fingerprint ridge contact pressure $\quad \mathrm{Pa}$

$\hat{p} \quad$ normalised mean fingerprint ridge contact pressure

$p(r) \quad$ finger pad pressure radial distribution $\quad \mathrm{Pa}$ 


$\begin{array}{lll}\bar{p}_{\text {asp }} & \begin{array}{l}\text { mean apparent contact pressure in an asperity } \\ \text { contact state area }\end{array} & \mathrm{Pa} \\ \bar{p}_{\text {crit }} & \begin{array}{l}\text { critical apparent contact pressure at which a } \\ \text { transition to a full contact state occurs }\end{array} & \mathrm{Pa} \\ \bar{p}_{\text {re }} & \text { mean real contact pressure } & \mathrm{Pa} \\ \bar{p}_{\text {trans }} & \quad \begin{array}{l}\text { mean apparent contact pressure in a full contact } \\ \text { state area }\end{array} & \mathrm{Pa} \\ r & \quad \text { radial distance from contact centre } & \mathrm{m} \\ R_{a s p} & \text { radius of an asperity tip } & \mathrm{m} \\ R_{f p} & \text { effective radius of a finger pad } & \mathrm{m} \\ w_{a s p} & \text { width of the texture asperity of feature } & \mathrm{m}\end{array}$

\section{GREEK SYMBOLS}

\begin{tabular}{lll} 
symbol & description & units \\
\hline$\alpha$ & pressure coefficient & - \\
$\beta$ & viscoelastic hysteresis loss fraction & - \\
$\delta$ & total micro-displacement of contacting surfaces & $\mathrm{m}$ \\
$\varepsilon$ & compressive Hertzian strain & - \\
$\eta$ & strain index for secant modulus & - \\
$\lambda$ & spacing between texture asperities & $\mathrm{m}$ \\
$\mu$ & coefficient of friction & - \\
$\mu_{\text {adh }}$ & $\begin{array}{l}\text { interfacial adhesion coefficient of friction } \\
\text { component }\end{array}$ & -
\end{tabular}




\begin{tabular}{|c|c|}
\hline$\mu_{\text {def }}$ & hysteresis coefficient of friction component \\
\hline$\mu_{\text {ref }}$ & $\begin{array}{l}\text { coefficient of friction measured for a reference } \\
\text { flat sample }\end{array}$ \\
\hline$\mu_{t e x}$ & $\begin{array}{l}\text { coefficient of friction measured for a textured } \\
\text { sample }\end{array}$ \\
\hline$\tau$ & interfacial shear strength \\
\hline$\tau_{0}$ & intrinsic interfacial shear strength \\
\hline$U_{m}$ & Poisson's ratio of a material \\
\hline$U_{s}$ & Poisson's ratio of skin in micro-scale contact \\
\hline$\varphi$ & load coefficient for secant modulus \\
\hline$\psi$ & real to fingerprint ridge contact area ratio \\
\hline$\psi_{s}$ & $\begin{array}{l}\text { real to fingerprint ridge contact area ratio } \\
\text { obtained from the numerical model }\end{array}$ \\
\hline
\end{tabular}




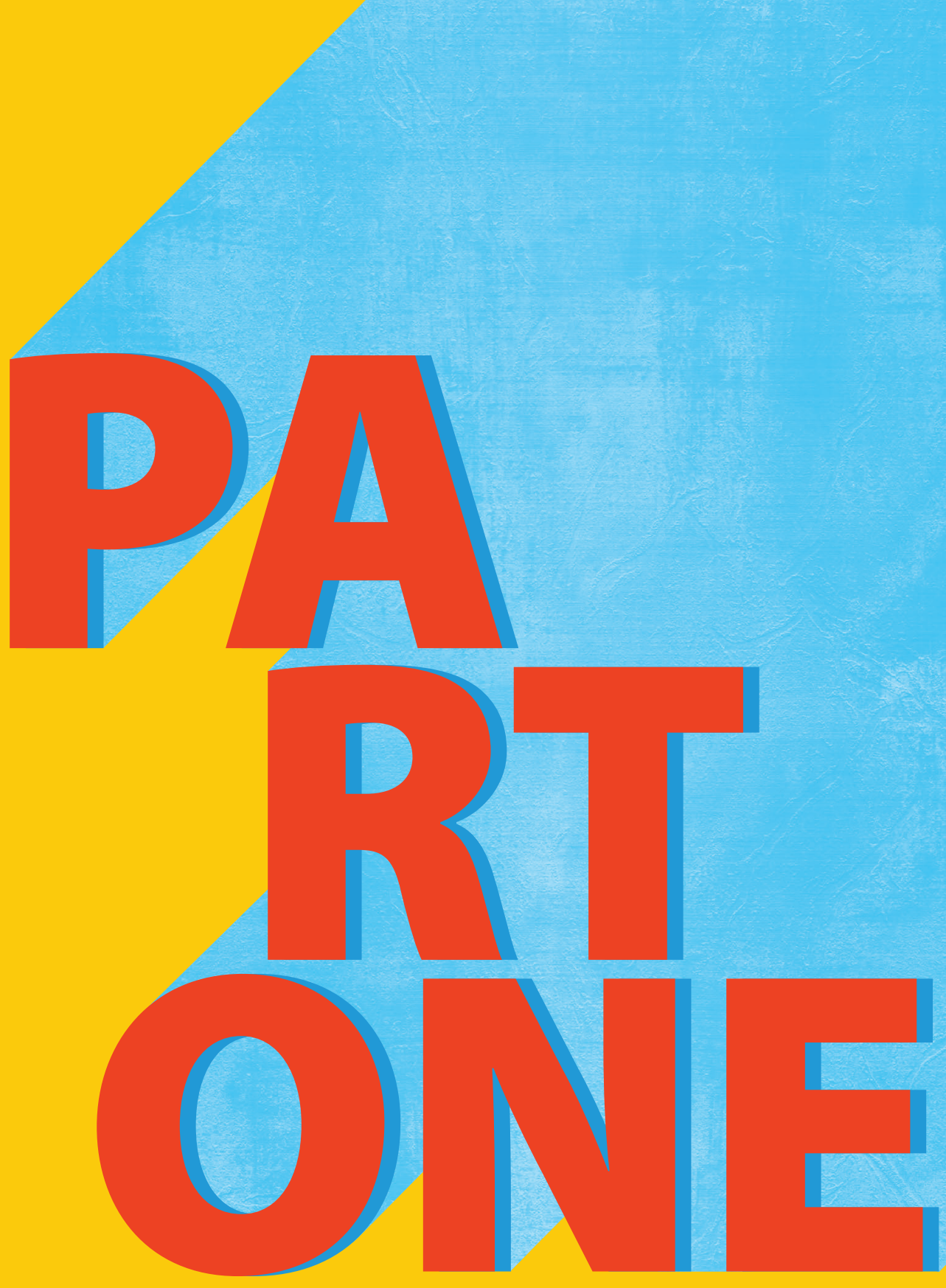




\section{INTRODUCTION}

\subsection{TACTILE FRICTION IN DAILY LIFE}

Probably, you have picked up and opened this book just a moment ago. It seems as a simple interaction with a known physical object, and yet, this action consists of several comprehensive phases [1]. First, the object, its size and position are visually recognised, and a course of action is being formed. Then the intention is executed: the arm reaches to the object, pre-shaping the hand with anticipation for a grasp. At last, fingers reach the object and the information obtained through touch, also called haptic perception, is added to the visual feedback. Haptic perception helps to correct the grasp and perform a precise movement based on the weight, centre of mass and other external forces acting on the object.

While you are holding an object, a constant control loop is maintained to ensure a stable grip. It includes stabilisation of inertial forces in case of movement, vibration damping, and slip detection. Reaction to a change in the system must be quick to prevent a collapse. By sliding a force probe against a finger, Johansson and Westling [2] report, e.g., an average reaction time to slippage of $74 \pm 9 \mathrm{~ms}$, after which the applied normal load is increased instinctively by the participant. Winstein et al. [3] observed similar delay between a change of an object weight and grip force adjustment. 
Haptic feedback combines kinaesthetic and tactile perception, where the first is an awareness of the body and limb position and the latter is an ability to receive information through skin stimulation. Slippage is only one of the effects that can be recognized through touch and friction. Tactile perception also provides such information about an object as its hardness, temperature, roughness, and stickiness [4,5]. The diversity of perceived properties expressed in attributes relates to the skin structure, which embodies a range of various receptors and nerve endings.

In high quality products special attention is paid not only to functional and visual design, but also to tactile aesthetics, so they elicit certain emotional responses [6]. The design solutions are generally found through panel testing [7], in which a variety of possible choices, e.g., replicas of different wood textures [8], is compared. Understanding the mechanics behind perception can reduce the number of samples, modify existing or even fully design the surfaces with a required response.

\subsection{FINGER PAD STRUCTURE AND SKIN PROPERTIES}

As shown schematically in Figure 1.1, human skin has three layers: the surface skin layer, the epidermis; the connective tissue layer, the dermis; and the innermost layer mainly composed of fat cells, the hypodermis. The epidermis or epithelial layer is the outermost layer of the skin and provides general protection to the body. It is mostly composed of keratinocytes, which move from the basal layer upwards, where they dehydrate, die, and get separated from the skin. As these cells migrate towards the surface they flatten and adhere together making a stratified scalelike structure called stratum corneum (SC). The thickness of the epidermis varies depending on the anatomical location from about $40 \mu \mathrm{m}$ on the eyelids [9] up to $800 \mu \mathrm{m}$ on the finger pads [10]. 


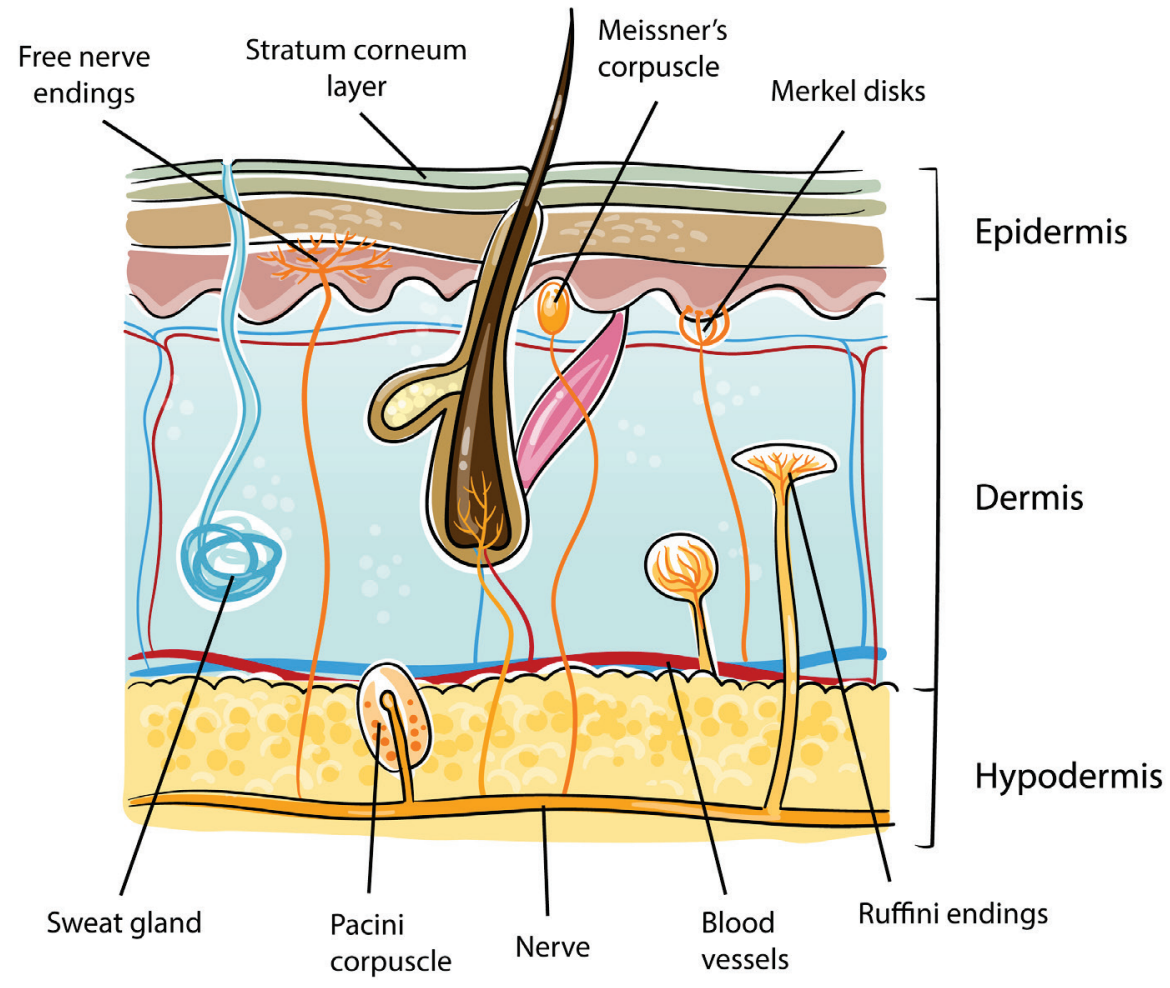

Daniela Barreto / Shutterstock.com

FIGURE 1.1 General structure of the skin.

Finger pads are covered with papillary ridges, which are known for their unique and permanent pattern as fingerprints. While there is no hair or sebaceous glands, fingerprint ridges are densely covered with sweat ducts with over 300 units per $\mathrm{cm}^{2}$ [11,12]. However, sweat does not serve for a cooling purpose, but forms a thin lubrication film between surfaces, affecting friction [12]. Adams et al. [13] proposed that presence of sweat softens the outer skin layer and increases the contact area with a surface 
as a result. This effect of moisture occlusion on the increase of the ridge contact area was observed by other researchers [14,15].

Evaluation of skin mechanical properties is problematic due to its layered structure, anisotropic behaviour, and the fact that it is a living tissue. Furthermore, its properties change between individuals and depend on age, gender, and environmental conditions [16-20]. Measurement methods are divided into in vitro and in vivo if the work is performed outside a living organism or within it, respectively. While the first approach allows to study various tissues separately, these results cannot be easily translated to the in vivo state. Edwards and Marks [21] described commonly used techniques, such as uniaxial and torsional extensometry, indentation, suction and wave propagation, which remain relevant to this day. Indentation measurements are considered the most appropriate while investigating friction behaviour. A detailed review on this technique and results interpretation was presented by van Kuilenburg et al. [20].

Indentation measurements are usually performed on the volar forearm skin where hair is not present, and the skin has uniform structure with relatively low roughness. Stratum corneum thickness in this anatomical location varies between 10 and 40 microns [22-24]. It is significantly thinner than on a finger pad, where it could reach 200-350 microns $[10,11,13]$. Therefore, the influence of the harder SC layer is reduced and the reported in vivo elastic modulus is found to vary between 5.7 and 40 $\mathrm{kPa}$ [25-29]. In vitro SC indentation measurements show elastic modulus higher by more than two orders of magnitude: 1 MPa to 1 GPa [22-24]. Mechanical properties of the finger pad skin are harder to assess due to the curvature and fingerprint ridge pattern. With the higher thickness of finger pad SC elastic modulus is expected to be higher. Abdouni et al. [16] used a glass indenter with a curvature radius of $2.5 \mathrm{~mm}$ and an air blast system to measure the elastic moduli, which was in the range between 10 $\mathrm{kPa}$ and $100 \mathrm{kPa}$. Cornuault et al. observed similar effective elastic 
modulus of $70 \pm 20 \mathrm{kPa}$ [18]. Wang and Hayward [30] tested finger pad skin in the tangential direction by stretching and shearing. They calculated the mean effective modulus between about 1 and $4 \mathrm{MPa}$ along and across the ridges.

Skin contains a variety of receptors, which transduce an external stimulus into an electrical impulse. Usually, four receptors related to mechanical stresses are distinguished: Meissner, Merkel, Pacini and Ruffini-like endings, see Figure 1.1. Pads of the fingers have a notably high density of Meissner and Merkel receptors, which are located under the epidermis layer and responsible for perception of dynamic spatial deformations and static forces, respectively. It estimates to about 17000 mechanoreceptors total in one hand [31]. The brain not only receives and processes the information from the nerve endings excitations, but also makes an unconscious prediction to perform a corrective action [32].

\subsection{PERCEPTION AND FRICTION}

Discrimination of a surface requires relative movement between object and fingers, often referred to as active tactile exploration. The exploratory movement depends on object shape and the general purpose of the motion [33-35]. Presumably, it allows to activate various classes of mechanoreceptors and obtain more information [36]. Hollins and Risner [37] performed a number of perception tests with sandpaper and noticed that for particle sizes below $100 \mu \mathrm{m}$ perception was significantly degraded without relative sliding.

Surface roughness and friction are considered to be the most important factors in surface discrimination [38]. Skedung et al. [39] compared perceived paper coarseness with its measured roughness and reported a positive correlation. Robles-De-La-Torre and Hayward [40] used an 
intermediate manipulator between surfaces and a finger and modified the forces during sliding over geometric cues. They concluded that force related information can overcome the perception of surface geometry. Barrea et al. [41] studied slippage detection and found that with reduction of friction from 1 to 0.3 for a flat sample, participants could no longer recognise if slippage occurred.

Yoshioka et al. [42] proposed participants to explore various surfaces, first with a finger and then with a probe, and compared the results of perception tests. Roughness evaluation was similar, but hardness and stickiness ratings differed between scanning methods. They explained it by vibratory power perceived with mechanoreceptors. Zhou et al. [43] also reported that higher vibration magnitude benefited the recognition of sandpaper samples. Fagiani et al. investigated influence of finger vibration in a series of elaborate studies [44-48]. Measured vibration frequency corresponded to textured surface only if its wavelength was below the wavelength of a fingerprint; in other words, the finger acts as a low pass filter with a specific cut-off frequency.

The relation between perception and friction was further studied with deterministic surfaces. Through testing of grooved and isotropic surfaces Cesini et al. [49] concluded that surface discrimination is influenced more by vibration spectra than its roughness. Skedung et al. [50] and Arvidsson et al. [51] studied surfaces produced by wrinkling with a wavelengths between $300 \mathrm{~nm}$ and $120 \mu \mathrm{m}$. They report correlation between roughness and slipperiness sensations and measured friction coefficient. Friction increased with a contact area between skin and polymeric surfaces. It was found to be higher for textures with the wavelengths above $60 \mu \mathrm{m}$ and below $1 \mu \mathrm{m}$, which was later confirmed with a contact model presented by Duvefelt et al. [52]. A wide range of pillar textures were investigated by Massimiani et al. [53] and Faucheu et al. [54]. Although a correlation between volunteer preferences and measured friction forces is not found, 
they confirm that textures with feature spacings below $160 \mu \mathrm{m}$ are considered smooth or slippery by a test group and are uniformly preferred over the others.

\subsection{SCOPE, APPROACH, AND LIMITATIONS}

\subsubsection{Scope of the thesis}

Information received through touch influences the perception of the surface physical properties, grip performance and slippage detection. Understanding of the mechanisms behind tactile perception and its deliberate incorporation into surface design can improve a vast variety of products from sport and medical equipment to consumer products and packaging.

Within this scope, the aim of the current work is to control and enhance tactile perception through modification of the frictional behaviour by surface topography design.

Production technology with which designed surface topography can be manufactured at an industrial scale includes a range of machining, moulding, and forming processes. Machining is based on material subtraction and its cost is directly related to the amount of removed material and time needed. Processes such as electrical discharge machining (EDM), chemical etching [55], or laser surface texturing (LST) [56] are suitable for texture design and can produce sub-micron resolution features [57], for steel in particular. While direct application of these techniques to engineering plastics is the subject of research, see e.g. [58], it currently remains too expensive and time-consuming for mass production. Injection moulding and hot embossing can be highlighted among other suitable manufacturing techniques due to their lower cost. Both of these processes are capable of transferring microscale textures 
on polymer materials in mass production [59-61]. While injection moulding gives more possibilities in shaping a part, roll-to-roll (R2R) embossing excels at continuous and, therefore, cheap manufacture of functional foils: filters, films, sensors [62], textiles, packaging and print media.

The latter makes hot embossing a good candidate for upscaling a production of textures with enhanced tactile performance. To develop the techniques for surface structuring, investigate the viable pattern designs and determine manufacturing requirements for such textures a collaborative project ${ }^{1}$ was funded by the INTERREG V-A DeutschlandNederland MOVERO program². The designed textures for tactile friction were first produced on embossing cylinders with the direct laser surface texturing by Schepers GmbH \& Co. KG (Vreden, Germany) and then transferred onto polyethylene foils through the R2R hot embossing process by SAUERESSIG GmbH \& Co. KG (Vreden, Germany). Manufacturing processes and surface designs were further optimised to achieve continuous production and maintain full texture transfer.

\subsubsection{Tribological system approach}

The object of the current study, tactile friction, is a response to the finger pad moving over a surface. A well accepted working method in tribology - the science and technology of interacting surfaces in relative motion [63] is the systems approach [64,65]. From this method, a tribological system

\footnotetext{
${ }^{1}$ Project partners: DLR-Institut für Vernetzte Energiesysteme e. V, Mikrobiologisches Labor Dr. Michael Lohmeyer GmbH, Universiteit Twente, Schepers GmbH \& Co KG, SAUERESSIG GmbH \& Co. KG, Irmato, Kamp Coating Apeldoorn BV, Materiomics, Morphotonics B.V., TAFH Münster GmbH, Duropanel BV, FMI Industrial Automation B.V., Matthews International $\mathrm{GmbH}$

${ }^{2}$ https://www.deutschland-nederland.eu/en/project/movero
} 
can be constructed for the finger pad - surface interaction, showing the related system components and the operational conditions. The primary function of the resultant system is the generation of information for touch perception through sliding. Energy losses due to the sliding interaction produce an output in the form of tactile friction and wear.

On the macroscale the tribological system consists of a finger in contact with a product surface as schematically represented in Figure 1.2. System output, i.e., tactile friction and wear, depend on operational conditions: environment, applied loads, sliding velocity, inclination angle, and component parameters: mechanical properties of each body, their structure and topography. An important distinction of the tactile tribological system is that finger properties cannot be directly controlled because they are related to another system - the human body. Skin properties vary, e.g., with time, temperature, and humidity, but in a steady environment skin reaches a stable state within a relatively short period of time. An acclimatisation time between 10 and 30 minutes is considered sufficient to stabilize skin properties [16,24,66-68].

Macroscale system

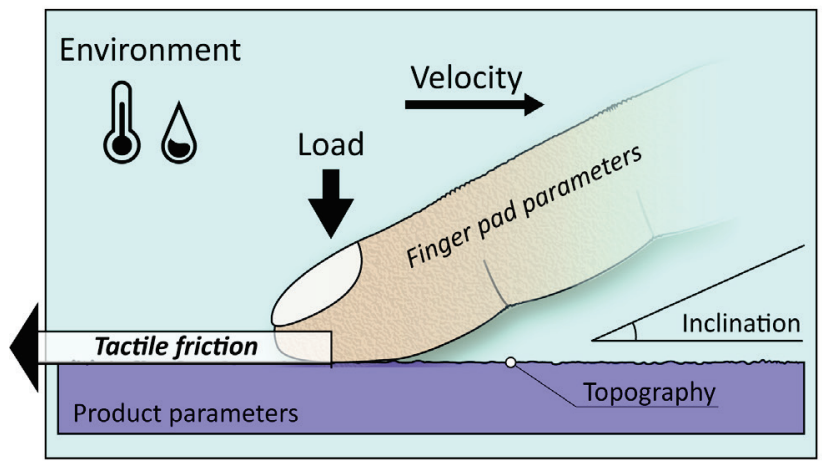

Microscale system

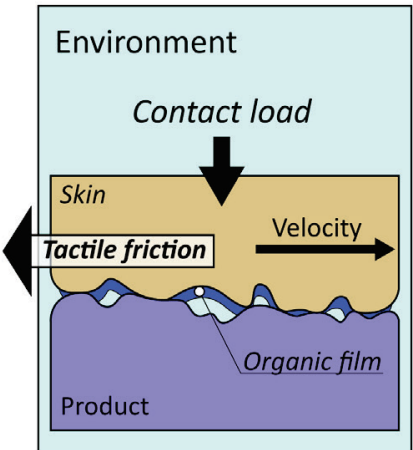

FIGURE 1.2 Tactile tribological system on (left) macro- and (right) micro scales. Parameters written in italic are related to a different system (human body) and cannot be controlled directly. 
The macroscale system can be considered at a lower coupled level. System on a micrometre length scale is represented by two contacting surfaces with an organic film between them (Figure 1.2). This lower-level system has a reduced number of parameters, but they are related to the macroscale system. For example, contact load is no longer a directly controlled parameter, but a product of applied load, finger pad properties and its fingerprint topography. Vice-versa, the output friction of the microscale system constitutes a part of the total tactile friction.

\subsubsection{Systems approach and limitations}

In this thesis the described systems approach is utilised, and finger pad contact is considered as a multiscale or nested system. The tactile friction is studied through a controlled variation of parameters while the system output is measured on the macroscale. Afterwards, the observed changes in the system response are explained by the processes occurring on the lower rank system. The influence of the three directly controlled parameters is analysed: product elastic modulus, applied normal load and surface topography, while other system parameters are kept stable. Furthermore, the relationship between the discussed systems is investigated by changing the finger parameters. The latter was achieved by performing measurements with a group of volunteers. Wear, as an output of the tribological system, is set outside the scope of the work. 


\subsection{THESIS LAYOUT}

The thesis consists of two parts: Part I is a structured summary of the performed research and Part II contains the research papers.

Part I includes six chapters, which follow a systematic approach.

Chapter 1 serves as an introduction to this work and presents the scope of the research, its practical purpose and general approach.

Chapter 2 describes the state of the art related to the mechanical behaviour of the finger pad, contact area and available predictive models. It is concluded with research gaps from which the research questions are formulated.

Chapter 3 presents a combined contact model developed to estimate the contact area between a finger pad and a microtextured surface. It is concluded with a parametric sweep calculation results with the purpose to connect texture dimensions to frictional behaviour.

Chapter 4 presents tactile friction measurements against a range of textures and explains the observed trends based on the introduced model and contact area differences.

Chapter 5 proposes a foundation for a texture design map aimed at relating texture dimensions with tactile friction and perception effects.

Finally, the conclusions of the current work and an outlook for future developments in the design and engineering of tactile surfaces are provided in Chapter 6. 


\section{FRICTION AND CONTACT MECHANICS}

\subsection{TACTILE FRICTION}

\subsubsection{Two-term friction model}

Friction can be defined as the resisting force tangential to the common boundary between two bodies when, under the action of an external force, one body moves or tends to move relative to the surface of the other [63]. In a system where one of the bodies is a human finger pad, friction is often referred to as tactile friction.

Tactile friction is considered to be composed of two terms based on the type of interaction, i.e., adhesion and deformation components [26]:

$$
F_{f}=F_{a d h}+F_{d e f}
$$

The interfacial adhesion component can be represented as a product of interfacial shear strength $\tau$ and real contact area $A_{r}$ :

$$
F_{a d h}=\tau A_{r e}
$$

Tactile friction is considered to be dry in the absence of a lubricant or other liquid. However, the skin of a finger pad is covered by sweat ducts [11] and, as such, a thin organic film is always present between the 
surfaces. Adams et al. [69] proposed an interfacial shear strength $\tau$ to be dependent on the mean real contact pressure $p_{r}$ as:

$$
\tau=\tau_{0}+\alpha p_{r}
$$

where $\tau_{0}$ is an intrinsic interfacial shear strength and $\alpha$ is a pressure coefficient.

The adhesion component remains predominant in a dry contact with relatively smooth surfaces [52,70,71]. Tomlinson [67] estimated that only about $3.9 \%$ of friction is related to bulk deformation of the finger and less than $0.2 \%$ for fingerprint ridges' deformation. In contact with soft materials, part of the energy is lost during material compression, which is referred to as hysteresis losses. It leads to an increase of the deformation component and can be estimated based on Greenwood and Tabor model [72]:

$$
F_{d e f}=\beta\left(\frac{9}{128 R_{f p}}\right)^{\frac{2}{3}}\left(\frac{F^{4}}{E_{0}^{*}}\right)^{\frac{1}{3}},
$$

where $\beta$ is a viscoelastic loss fraction, $R_{f p}$ is a finger pad equivalent radius, and $E^{*}$ is a reduced elastic modulus.

Among other factors that contribute to the friction forces are the capillary adhesion and formation of liquid bridges, which appear in the presence of moisture. Those effects are considered plausible for tactile friction by multiple studies [73-75]. Essentially, an adsorbed water film on the surfaces creates a meniscus between surface asperities pulling them together and thus increases the effective contact area. Such capillary effects increase for elastic surfaces with high surface energy, low roughness, and low water film thickness [76]. Tomlinson et al. [77] analyse capillary force contribution to the finger pad friction with 
addition of moisture. They find it hard to prove that capillary adhesion occurs and conclude that water adsorption by the skin and its subsequent plasticising is the most likely reason for the observed increase of friction.

\subsubsection{Tactile friction against stochastic surfaces}

Tactile friction does not follow Amontons' law, which prescribes a constant friction coefficient independent of normal load. Derler et al. [70] discuss friction measurements for a finger sliding against smooth and rough glass surfaces under dry and wet conditions. The roughness of the surfaces is created by sandblasting thus creating a stochastic distribution of the surface asperities. For dry sliding against the smooth sample the coefficient of friction follows a power law function $\mu=k F^{n-1}$ with observed exponents $n-1$ between -0.96 and -0.79 . Friction for the rough sample remains independent from the normal load but only in the absence of water. When deionised water is added to the contact, Derler et al. [65] show that the friction values increase and becomes load dependant, with the exponents between -0.80 and -0.63 . This behaviour is explained by predominant adhesion friction and water uptake by the skin, which increases its compliance and, consequently, contact area. Experimental results of Tomlinson et al. [78] confirm the linear relationship between friction and normal forces for surfaces with various random roughness. An initial increase of surface roughness has a negative correlation with the coefficient of friction [79]. However, surface roughness cannot be used as a predictor of friction by itself. Despite the similar average surface roughness, around $5 \mu \mathrm{m}$, abrasive papers show different frictional behaviour than the rough glass [68]. This is attributed to the conical shape of the asperities on the abrasive paper, which introduces skin ploughing and abrasion effects [80]. 


\subsubsection{Tactile friction against deterministic surfaces}

Most of the deterministic surfaces that are studied in contact with a finger pad can be separated by three texture types depicted on Figure 2.1: dimple matrix, bump asperity matrix and parallel-ridged surfaces, with the latter two being predominant. The largest rectangular ridged surfaces are studied by Tomlinson et al. [81] with a range of ridge widths between $1 \mathrm{~mm}$ and $5 \mathrm{~mm}$ and spacings up to $16 \mathrm{~mm}$. Macroscopic geometry resulted in uneven friction curves with a delta between maximum and minimum values increasing with ridge spacing and heights. In another work Tomlinson et al. [82] proposes a model for an interlocking component of friction, which arises from fingerprint ridges 'climbing' over inclined surfaces. The interlocking component is noticeable for the ridge heights from $33.5 \mu \mathrm{m}$ and becomes predominant starting at $105 \mu \mathrm{m}$ height. Reduction of ridge spacing to about $100 \mu \mathrm{m}$ and their height below $30 \mu \mathrm{m}$ results in reduction of friction, perceived stickiness and bidirectional sliding difference due to hysteresis effects in comparison to the reference flat surface $[83,84]$. Skedung et al. [50] studied a range of samples with spacings below $100 \mu \mathrm{m}$ and report a reduction of friction coefficient down to about 0.4 for samples with spacing between 20 and $40 \mu \mathrm{m}$. Friction started to increase with further reduction of texture ridge
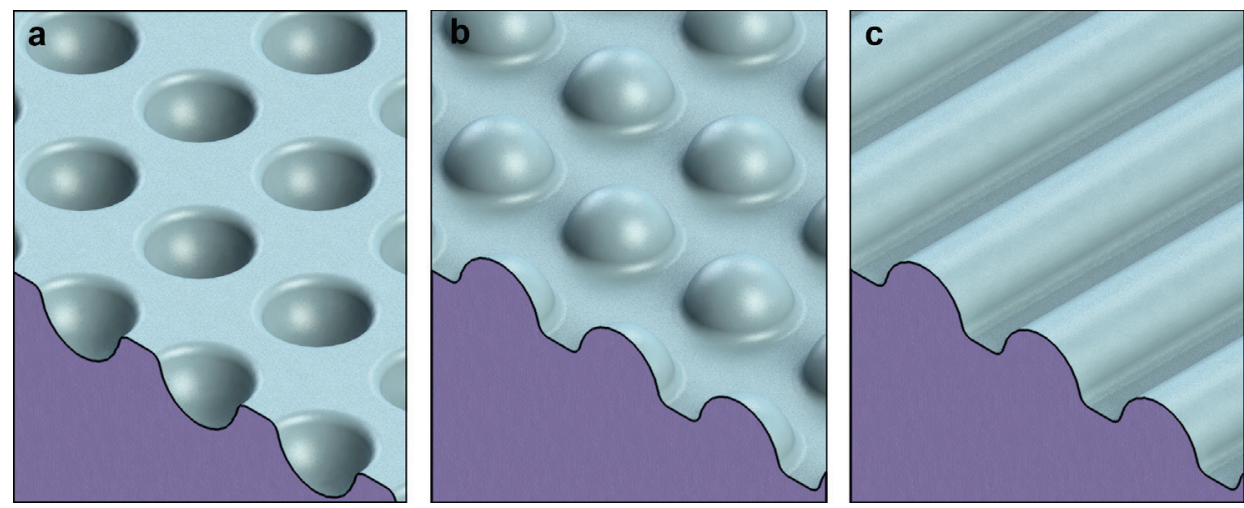

FIGURE 2.1 Dimple (a), bump (b) and parallel ridge (c) texture layouts based on a similar circular feature profile. 
spacing. Based on these results Duvefelt et al. [52] proposed a Westergaard based contact model.

Darden and Schwartz [85] investigated a frictional response during sliding over Braille print features located at various distances from each other. They report a hysteresis deformation reflected in increased friction when a finger slides over a feature. Childs and Henson [86] studied screen printed surfaces with different textured areas and spacings between 0.4 and $1.4 \mathrm{~mm}$. Interestingly, they reported that several textures remained in an asperity contact state with the skin, even though there was no significant reduction in the related friction coefficient. Massimiani et al. [53] recently performed elaborate research on the perceptive role of mechanical stimuli with a large variety of pillar bump textures. They conclude that friction does not affect the texture perception for the tested samples, however the samples with lateral spacing below $160 \mu \mathrm{m}$ were uniformly considered smooth by participants. The finest texture dimensions were tested by van Kuilenburg et al. $[87,88]$ with asperity radii between 1 and $20 \mu \mathrm{m}$ and spacings between 20 and $200 \mu \mathrm{m}$. They obtained the lowest friction with an asperity radius of $2 \mu \mathrm{m}$ and $20 \mu \mathrm{m}$ spacing and predict a contact area correlation with surface texture dimensions based on a modified Hertzian contact model. Furthermore, the authors conclude that a minimum friction coefficient can be achieved by changing asperity density.

Tactile friction measurements are often performed at a few normal loads and do not represent contact area development as a function of applied force. Manual finger pad sliding introduces large deviations in measured data due to an adhesive contact and hysteretic finger bulk deformation. 


\subsection{CONTACT MODELLING}

Finger contact can be modelled at multiple length scales: macroscopic deformation of a finger pad, mesoscopic displacement of fingerprint ridges and microscopic skin contact with surface asperities (Figure 2.2). Further in the text the corresponding contact areas for these dimensional scales are referred as gross, ridge and real, respectively. Decoupling of these length scales allows development of predictive models focused on specific aspects of finger contact mechanics. However, estimation of the real contact area requires consideration of all three scales.
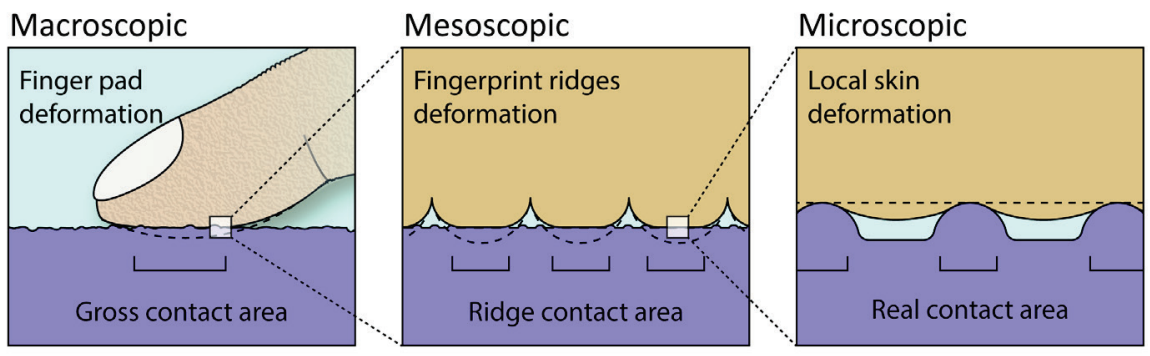

FIGURE 2.2 Three considered length scales of finger pad contact, corresponding skin deformation and contact areas.

\subsubsection{Macroscopic contact}

Under a static normal load, the finger pad deforms up to a few millimetres due to the presence of a softer hypodermis tissue between epidermis and bone. Determined by the structure of the finger, its deformation and stiffness vary with inclination angle to the surface plane. For example, a change of the angle from $0^{\circ}$ to $45^{\circ}$ reduces the deformation on average from $3 \mathrm{~mm}$ to $2 \mathrm{~mm}$ at $4 \mathrm{~N}$ normal load [89]. This is explained by a distal phalanx bone, against which a dermis is compressed and its layer thinning towards the fingertip. Furthermore, in the same research Serina et al. showed that an increase of loading rate can lead to a noticeable rise 
of stiffness and mentioned a time required for a complete tissue recovery after load removal. They conclude that an appropriate analytical model should include such factors as finger shape, elastic and viscoelastic properties of the skin.

Contact of spherical bodies in general can be predicted by a classical Hertzian model. The circular contact area for a sphere on a plane is then represented by:

$$
A=\pi\left(\frac{3 F R}{4 E^{*}}\right)^{\frac{2}{3}}
$$

where $F$ is the applied normal load, $\mathrm{R}$ is the radius of a sphere and $E^{*}$ is the reduced Young's modulus of the interacting surfaces. The fingertip can be considered as a semi-ellipsoid or an equivalent hemisphere with an effective radius $\mathrm{R}$. This approach allows to estimate skin elasticity based on deformation [18] or gross contact area assuming skin properties $[48,83,84,87,90-92]$. The Hertzian contact model predicts the contact area to increase with normal load as a power function with an exponent of 0.66 . However, various empirical studies find this value to be in the range between 0.1 and 0.5 based on the best power fit to the gross area measurements $[11,87,90,93,94]$. Furthermore, an increase of the finger pad stiffness with deformation leads to a change of the exponent. To represent this behaviour, Liu et al. [90] used two different power functions for (1) normal loads up to $2 \mathrm{~N}$ and (2) above $2 \mathrm{~N}$. The discrepancy between experimental fitted results and the Hertzian model is depicted in Figure 2.3. 


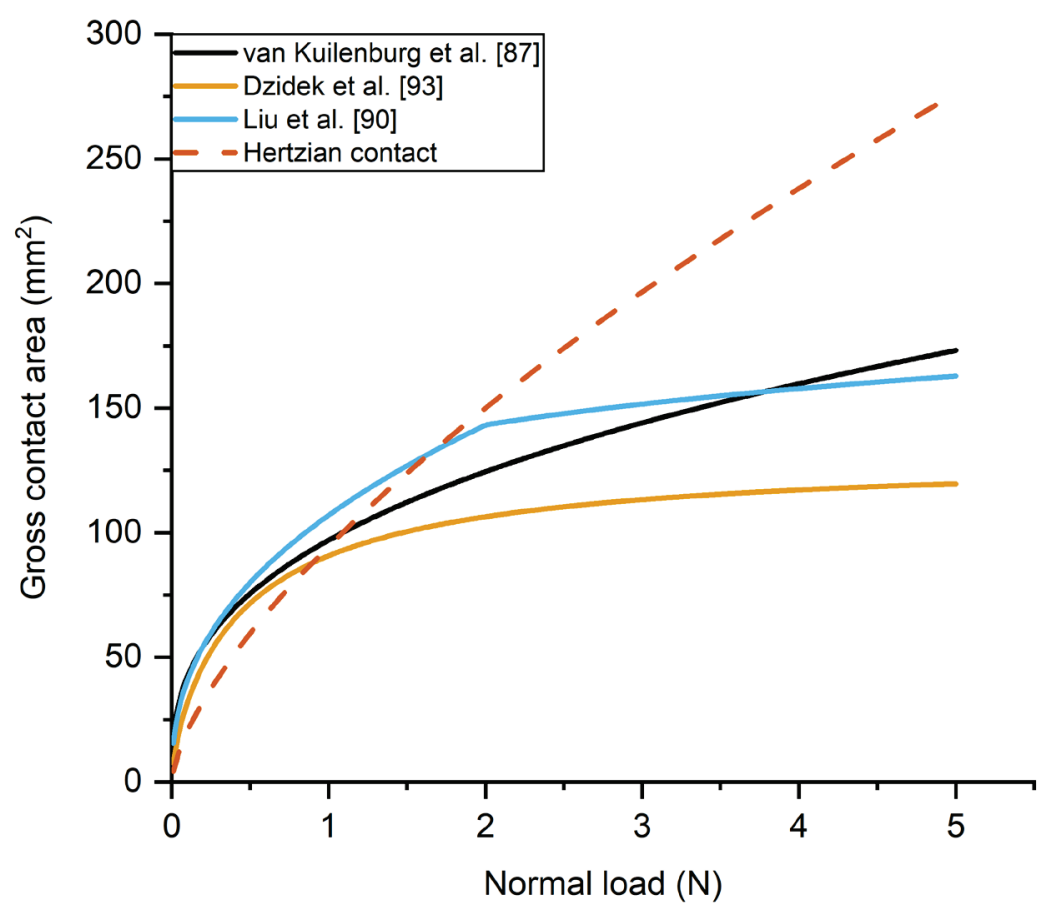

FIGURE 2.3 Comparison of the experimental power fits with Hertzian contact model. Hertzian contact calculated for skin elastic modulus of $50 \mathrm{kPa}$ and finger pad radius of $11 \mathrm{~mm}$.

The Hertzian model assumes small deformations and a small contact spot radius compared to dimensions of the bodies in the contact, which are not valid for a finger pad case. Moreover, skin stiffness and equivalent radius are dependent on the finger inclination angle. Those factors combined do not allow the use of the Hertzian contact model in its initial form for a wide range of normal loads. Macroscopic deformation is mainly governed by finger structure and its inner tissues, which allows to perform gross contact area measurements and use a power fit function as a prediction for an individual. 
In order to improve one of the Hertzian model aspects, particularly the change of the skin effective elastic modulus with deformation, Dzidek et al. propose an improved analytical model [93]. They notice that at very small displacements stratum corneum stiffness influences the measured skin elasticity. However, skin elastic modulus reduces rapidly and remains relatively stable at a value around $40 \mathrm{kPa}$ for displacements below $0.9 \mathrm{~mm}$. With further deformation skin becomes compressed against the distal phalanx bone which leads to the subsequent increase in the effective elastic modulus. Dzidek expresses this change through the power law function of the strain as:

$$
\frac{E_{s f p}^{*}-E_{f p}^{*}}{E_{s f p}^{*}}=(C * \varepsilon)^{\eta},
$$

where $E_{s f p}^{*}$ - reduced secant elastic modulus of the fingerpad, $E_{f p}^{*}$ reduced elastic modulus of the finger when no constraints apply, $C$ constraint coefficient, $\varepsilon$ - strain $\left(\varepsilon \in\left[0, \frac{1}{c}\right] ;\right)$ and $\eta$ - strain index. Dzidek et al. find the experimental data to be most accurately described by the strain index $\eta=3$ [93].

From this equation, a reduced secant elastic modulus $E_{s f p}^{*}$ can be expressed and substituted in Eq. 2.5 to receive a following form of the Hertzian equation:

$$
A_{g}=\pi\left[\frac{3 R_{f p} F}{4 E_{f p}^{*}(1+\varphi F)}\right]^{\frac{2}{3}}
$$

where $\varphi$ is a load coefficient and equals $\varphi=\frac{3(0.2 C)^{\eta}}{4 R_{f p}^{2} E_{f p}^{*}}$

Their results explain the exponent variance with a change in finger pad stiffness, which is in line with observations of Liu et al. [90]. They show 
that this approach remains valid for relatively large displacements (up to $1.6 \mathrm{~mm}$ ). Nonetheless, it still requires calculating the finger pad reduced elastic modulus $E_{f p}^{*}$, constraint coefficient $C$ and strain index $\eta$ from the stress-strain measurements, which limits the model applicability.

\subsubsection{Mesoscopic contact}

Deformation of the fingerprint governs a ridge contact area, which can be significantly lower than the gross contact. Depending on the normal load, the reported area fraction lays in the range from $10 \%$ to $70 \%[11,86,94,95]$. The fraction of the fingerprint ridge contact area varies with normal load; however, their relationship in literature is contradictory. Based on a number of measurements for six individuals Soneda and Nakano [94] reported a reduction of contact area ratio with normal load, while a directly opposite behaviour can be observed from the measurements of Childs and Henson [86]. Similar to the macroscopic contact, the fingerprint ridge contact area can be approximated for an individual by a power function of normal load with the exponent between 0.2 and 0.7 $[13,90,93,96]$. Maximum principal stresses are reached within fingerprint ridges [97] and their deformation with normal load should be considered in predictive models.

A straightforward assumption is to consider a trapezoidal ridge profile rather than a circular one, implying that their individual deformation is negligible. Following this approach, van Kuilenburg et al. [87] considered ridges of certain widths and densities, which resulted in constant ratio between ridge and gross contact areas. Similar consideration was adopted by Duvefelt et al. [52], who considered ridge contact to remain at 50\% of the gross contact area. As discussed previously, such an approach however results in limitations to the normal load range in which the model remains applicable. 
Rodriguez Urribarri et al. [95] applied a Westergaard solution for sinusoidal surfaces to calculate the fingerprint ridge contact area. Fingerprint structure is considered as a series of sinusoidal parallel ridges, each making a line contact with a flat surface. Optical coherence tomography (OCT) images are used to measure the fingerprint ridge wavelength, while the contact areas are calculated from the fingerprint scanner data. Effective elastic modulus of the skin calculated by this approach fell into the range between 0.05 to $0.3 \mathrm{MPa}$. Possible errors arise from the fact that a sinusoidal surface does not represent the fingerprint ridge structure and, therefore, underestimates both the calculated contact area and the ridge radius.

There are other models that can be adopted for a fingerprint ridge contact area calculation, some of them are presented in Figure 2.4. Compared with experimental measurements obtained in Paper A, an assumption of the constant area fraction shows a significant overestimation at normal loads below $2 \mathrm{~N}$, but provides a good approximation at higher loads. Representation of a fingerprint topography has the largest impact on calculation results. Models that assume a fingerprint as a sinusoidal profile (Figure 2.4a) predict lower ridge contact area than the models based on circular ridge profile (Figure 2.4b). Results obtained through a boundary element method (BEM) used a part of the measured fingerprint replica and its output is closer to analytical models with circular profiles. It restricts deformation of the ridges and provides the most accurate results in the range of normal loads between 0.5 and 2 N. Notably, all presented methods underestimate contact area at low loads, which can be compensated by inclusion of adhesive interaction, e.g., JohnsonKendall-Roberts (JKR) adhesive model. 


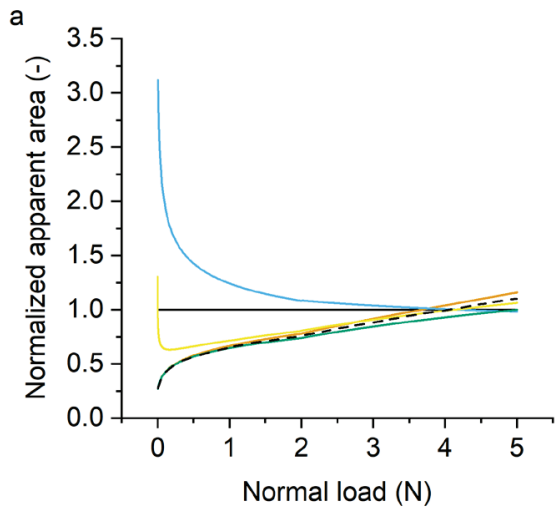

\begin{tabular}{|l|}
\hline Experimental \\
Sine surface - Westergaard \\
Trapezoidal - constant value of 0.5 \\
Cylinder equivalent - Hertzian \\
Cylinder equivalent - Hertzian + JKR \\
- - - Sine surface - numerical
\end{tabular}

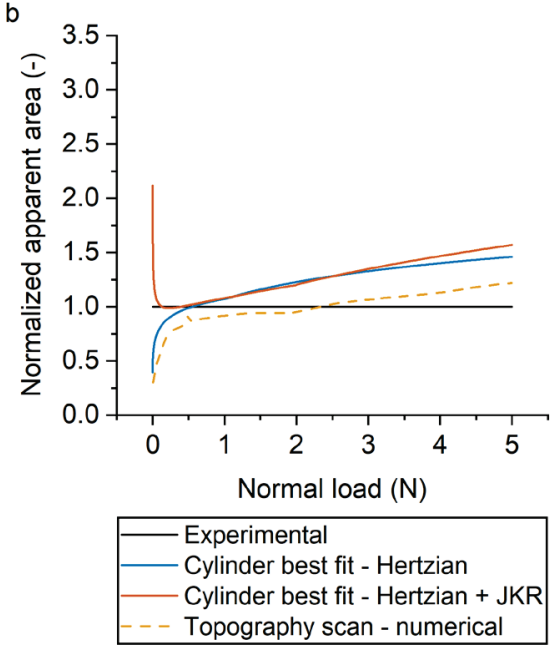

FIGURE 2.4 Comparison of the mesoscopic models. The calculations are normalized by experimental results to highlight the difference in resulting exponent. All models use the same force, gross area and ridge spacing inputs; an effective Young's modulus of $100 \mathrm{kPa}$ is assumed for the skin.

\subsubsection{Microscopic contact}

The sum of contact areas between skin and surface summits is considered the real contact area. The real or true contact area usually remains a small fraction of the fingerprint ridge contact and, while it cannot be fully defined, its decoupling is necessary to predict the mechanics on the micro- length scale. Direct measurements of the real contact area prove to be difficult due to the low pressures and presence of an intermediate sebum layer. Therefore, microscopic models are validated through friction measurements against deterministic surfaces. This approach allows to restrict maximum contact area and compare frictional behaviour between multiple texture designs. Depending on the texture type, parallel ridges or an asperity matrix, Westergaard or Hertzian models are applied in literature respectively. 
Duvefelt et al. [52] analysed friction measurements with 17 parallel ridge textures performed in a preceding perception study [50]. They used a Westergaard-based contact model to estimate contact area and deformation of the skin at the micro-scale and obtained a high correlation between the prediction and the experimental results. Rodriguez Urribarri et al. [95] adopted the same base model, but had different initial assumptions. They considered a contact between a fingerprint pattern and a parallel ridge texture equivalent to a bi-sinusoidal surface against flat. They concluded that such an approach is valid for skin elastic modulus in the range between 0.12 and $1 \mathrm{GPa}$, which is consistent only with the higher end of the values reported for an isolated stratum corneum at penetration depths below 1 um [22].

Surface textures composed of asperity bumps were studied by van Kuilenburg et al. [20,87,88]. They adopted a Hertzian model for individual asperities and proposed a relationship between texture dimensions and real contact area. However, Hertzian contact assumes no stresses outside of the contact width, which increases inaccuracy with asperity density. More importantly, it cannot describe the cases when skin deformation reaches the asperity height threshold and comes in contact with a base surface. 


\subsection{CONCLUSIONS}

The contact between the finger pad and engineering surfaces consists of a macroscopic scale, a mesoscale and a microscopic scale. The state of the art in contact modelling reveals that:

1. Hertzian contact model is best suited for single asperity skin contact and small deformations.

2. Hertzian model can be applied to describe gross contact area if used with a variable skin elastic modulus.

3. Westergaard-based contact models show good agreement for sinusoidal surfaces, but results in increasing errors for predicted deformation and friction for real engineering surfaces.

4. JKR and capillary forces are considerable at low normal loads. At normal loads above $0.5 \mathrm{~N}$ their contribution to tactile friction becomes negligible.

From the presented state of the art in Chapter 1 it is concluded that the human skin has a profound influence on tactile friction:

1. Skin is anisotropic and its reported properties vary in the order of several magnitudes based on the measurement method, probe radius and predictive model used for the calculation. It remains unclear what range of skin modulus of elasticity should be considered for the microscale contact modelling.

2. The difference in skin properties between individuals and its effect on friction remains an item of discussion in literature. Comparison of the trends is generally limited by the power function exponent, which is related to unknown finger pad parameters. 
For tactile friction the following can be concluded:

1. Tactile friction can be described by a two term friction model consisting of an adhesion term and an deformation term.

2. The adhesion component remains predominant for surfaces with low roughness.

3. Surface texture designs are able to change finger pad friction.

4. There is a variety of macro-scale asymmetric texture-designs, for direction dependent frictional behaviour, however, there were no attempts to create directional friction dependence with microscale textures.

Several publications report the skin reaching the surface valley, which leads to the rise in coefficient of friction. Contact models described in literature are based on analytical solutions and do not allow to model this effect at the level of microscopic contacts.

Based on the state of the art, the following steps are identified to address the existing research gaps:

- Develop a contact model which could predict contact area independent of the surface topography and/or contact state.

- Estimate the skin effective elastic modulus relevant for a contact with multiple asperities at the microscale.

- Analyse the tactile friction behaviour with a change in personal finger pad and skin properties with the aim of its normalisation.

- Investigate textures with directional friction behaviour based on microscopic contact and related contact area variation.

By addressing these research gaps, it is foreseen that a design map for surface design in tactile contacts can be constructed. 


\section{NUMERICAL METHODS}

\subsection{MICROSCOPIC CONTACT}

Skin contact with micro-texture features can take one of the three different states depicted in Figure 3.1. At initial contact two surfaces share only a small fraction of the total area, which depends on the normal load, the asperity radii and their lateral spacing. With increasing load, both surfaces deform until a contact transition occurs, i.e., the skin makes a contact with a valley between asperities. This deformation sets a boundary condition for the asperity contact state. Additional contact regions change the trend for the contact area - normal load function. Further subsequent rise of the applied force eventually leads to full contact with the texture in which the contact area reaches its potential maximum. After the full contact state at the microscale is reached, the
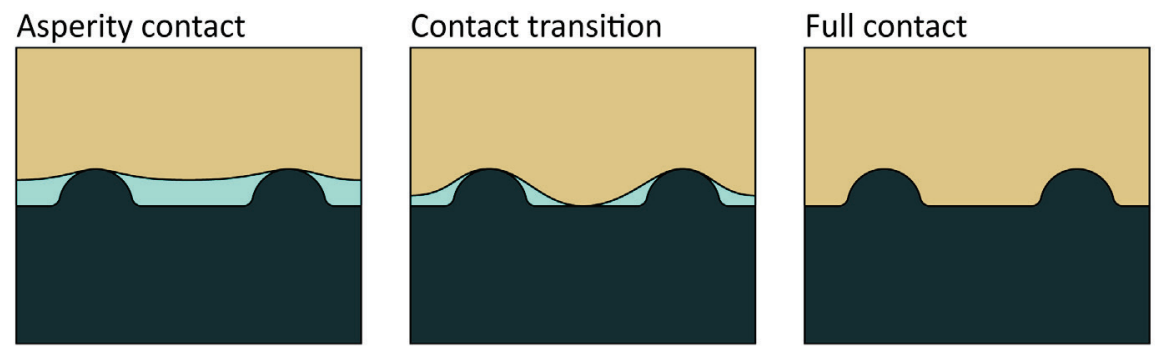

FIGURE 3.1 Microscopic tactile contact states with surface texture features. 
contact area will remain constant with further increase of the normal load.

The adhesive component of tactile friction is directly related to the contact area and from that relation it is assumed in this work that the contact state can be determined through friction measurements. Observation of the contact transition allows identification of the asperity and full contact states based on the friction values. This reference point is particularly useful for a finger pad contact because finger pad parameters are unique per person and affect the absolute friction measurements.

The asperity contact state and its modelling was discussed by van Kuilenburg et al. [87]. They applied the conventional Hertzian theory to investigate the influence of texture dimensions on finger pad contact area. Contact with each asperity was assumed independent of surrounding features and was considered as a sphere against flat surface case. The number of micro asperities in contact with the finger $N$ was calculated from the lateral spacing of the features $\lambda$ as:

$$
N=A_{f r} / \lambda^{2}
$$

Consequently, the mean normal force acting on an individual asperity $\bar{F}_{i}$ can be expressed through the normal load applied to the finger pad $F$ or the mean ridge pressure $\bar{p}$ as:

$$
\bar{F}_{i}=\frac{F}{N}=\frac{F \lambda^{2}}{A_{f r}}=\bar{p} \lambda^{2}
$$

The boundary condition for the application of the Hertzian model is defined by the contact transition. The critical force per asperity $F_{i_{c r i t}}$, at which the transition happens, can be calculated using surface parameters. 
The contact transition can be defined as a state, at which the combined micro-displacement of the surfaces $\delta$ equals the asperity height $h_{\text {asp }}$. This requirement can be expressed by the Hertzian approach as:

$$
\delta=h_{a s p}=\left(\frac{9}{16} \frac{F_{i_{c r i t}}^{2}}{E_{1}^{* 2} R_{a s p}}\right)^{\frac{1}{3}}
$$

where $R_{\text {asp }}$ is a radius of individual asperity and $E_{1}^{*}$ is a reduced Young's modulus for micro contact. The reduced elastic modulus $E_{1}^{*}$ can be calculated from:

$$
\frac{1}{E_{1}^{*}}=\frac{\left(1-v_{s}^{2}\right)}{E_{s}}+\frac{\left(1-v_{m}^{2}\right)}{E_{m}}
$$

where $v_{s}, v_{m}$ and $E_{s}, E_{m}$ are the Poisson's ratio and Young's modulus for the skin and the counter-surface material, respectively. Critical force per asperity required for the contact transition $F_{i_{c r i t}}$ is related to the normal load as given in Eq. 3.2 and reaches its maximum in the centre of the contact, at a point of the maximum pressure. This can also be visualised in Figure 3.3. Assumption of a spherical pressure distribution for the finger pad yields a coefficient of 1.5 for the mean asperity pressure $\bar{F}_{l}$, therefore:

$$
F_{i_{c r i t}}=\frac{3}{2} \bar{F}_{l}=\frac{3}{2} \frac{F_{c r i t}}{A_{\text {fr }}} * \lambda^{2},
$$

Solving the set of equations 3.3 and 3.5 for the applied normal load $F_{\text {crit }}$ gives the following relation to the system parameters:

$$
F_{c r i t}=\frac{8}{9} \frac{\sqrt{h_{a s p}^{3} R_{a s p}}}{\lambda^{2}} * A_{f r} * E_{1}^{*}
$$


While a simplified Hertzian contact can be used as a first approximation, it cannot be applied for precise calculations due to the high asperity density and contribution of the compressive stresses from the surrounding surface features. This approach overestimates the effect of the reduced elastic modulus and it overestimates the normal forces required for the contact transition to happen. Furthermore, calculation of contact area at full contact cannot be performed with the Hertzian model. This disadvantage can be overcome with a numerical boundary element method (BEM), as will be discussed in section 3.2.

\subsection{NUMERICAL METHOD FOR CONTACT STATE ESTIMATION}

The problem of stress distribution at any point in a semi-infinite isotropic and homogeneous elastic half-space was solved by Boussinesq [98] and is widely used in numerical contact models [99-101]. Therefore, the calculation of stresses on the micro-scale is performed by a numerical half-space contact model considering the skin as an ideal flat elastic surface. Boundary element methods permit the use of a discretised surface topography as an input obtained by, for instance, confocal microscopy.

Numerical simulation is performed for the microscale system, for example for a single asperity. Figure 3.2 depicts a typical example of the BEM simulation results performed for a texture with laterally spaced spherical features. Three contact states can be clearly defined by the contact area ratio and its rate of change as a function of the normal load. To find the total contact area of the finger pad, the normal forces acting on the asperity must be correlated with a load on the macroscale and the results over the whole macro contact must be summed up. Furthermore, 

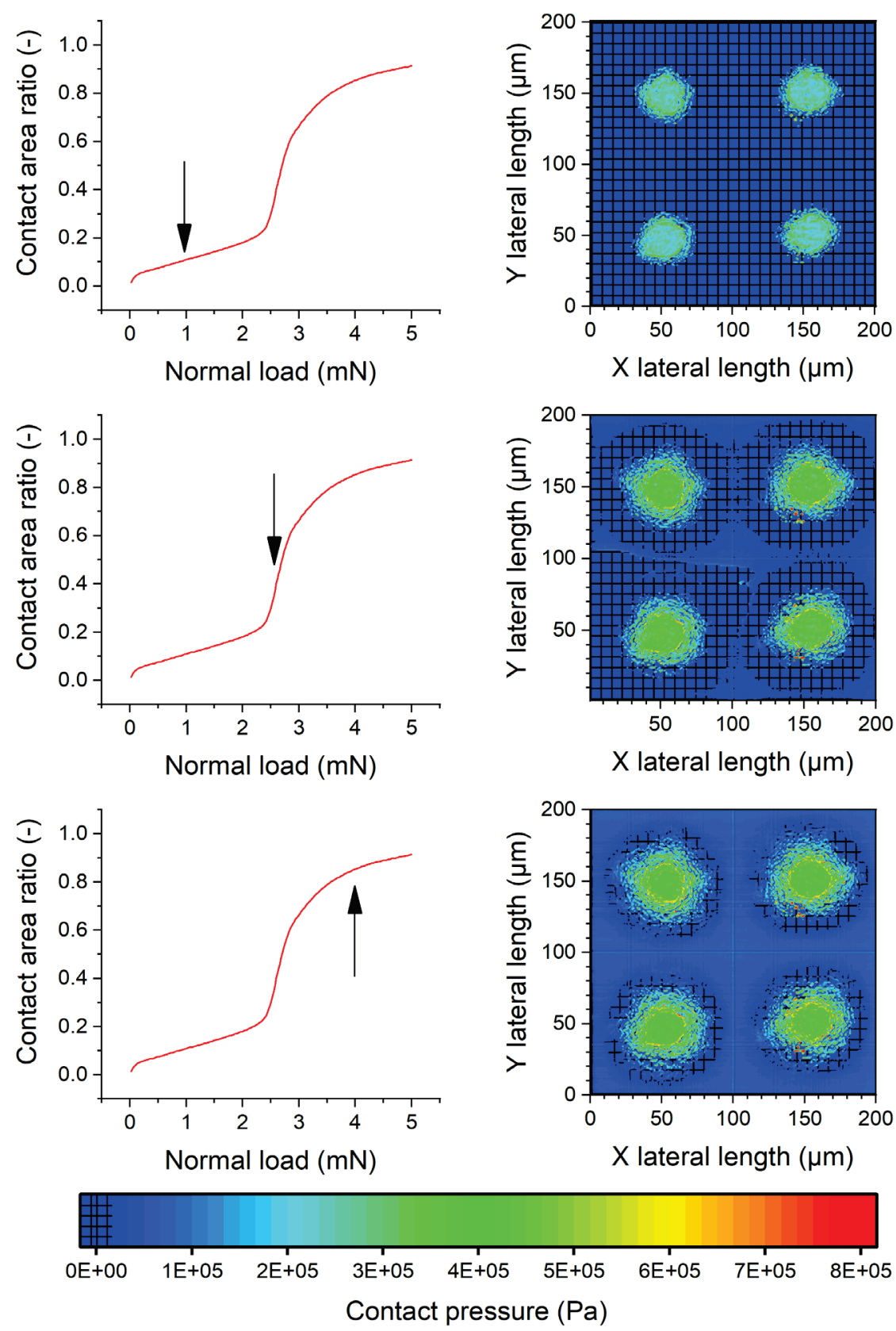

FIGURE 3.2 Typical BEM results for a textured surface with respect to the three contact states. The left column shows the real contact area ratio, the right - contact pressure distribution. 
finger pad pressure distribution affects local skin deformation and should be considered. The pressure gradient implies that transition effect on the macroscale is not immediate but develops gradually with increase of the normal load. Assuming a spherical pressure distribution for the finger pad, the actual pressure $p$ can be calculated for any point at distance $r$ from the contact centre if the mean pressure $\bar{p}$ and equivalent contact radius $a_{f r}$ are known:

$$
p(r)=\frac{3}{2} \bar{p} \sqrt{1-\frac{r^{2}}{a_{f r}^{2}}}
$$

Substituting the radii and pressure in the expression, it can be rewritten for the transition case as:

$$
F_{i_{c r i t}}=\frac{3}{2} \overline{F_{l}} \sqrt{1-\frac{A_{\text {trans }}}{A_{\text {fr }}}},
$$

where $A_{\text {trans }}$ is the fingerprint ridge contact area in the full contact state.

This notation allows to represent the fraction of the ridge contact area, which passed the transition point through the ratio of the asperity forces:

$$
\frac{A_{\text {trans }}}{A_{f r}}=1-\left(\frac{2}{3} \frac{F_{i_{\text {crit }}}}{\bar{F}_{i}}\right)^{2}, 0 \leq \frac{A_{\text {trans }}}{A_{f r}}<1
$$

The equivalent contact radius of the zone in full contact $a_{\text {trans }}$ can be expressed from the calculated ratio:

$$
a_{\text {trans }}=a_{f r}\left(A_{\text {trans }} / A_{f r}\right)^{1 / 2}
$$


The mean pressures in regions in full contact and in asperity contact state, $\bar{p}_{\text {trans }}$ and $\bar{p}_{\text {asp }}$ respectively, can be calculated through integration of the Hertzian pressure distribution equation (Eq. 3.7) over the corresponding areas:

$$
\begin{gathered}
\bar{p}_{\text {trans }}=\frac{1}{\pi a_{\text {trans }}^{2}} \int_{0}^{a_{\text {trans }}} \frac{3}{2} \bar{p} \sqrt{1-\frac{r^{2}}{a_{f r}^{2}}} * 2 \pi r d r \\
\bar{p}_{\text {asp }}=\frac{1}{\pi\left(a_{f r}^{2}-a_{\text {trans }}^{2}\right)} \int_{a_{\text {trans }}}^{a_{f r}} \frac{3}{2} \bar{p} \sqrt{1-\frac{r^{2}}{a_{f r}^{2}}} * 2 \pi r d r
\end{gathered}
$$

This approach allows to split finger pad contact into two zones as schematically shown in Figure 3.3. Starting from the asperity contact state, the mean asperity force for the finger pad is used for BEM simulation, i.e. $F_{i}=\bar{F}_{i}$. With increasing normal load, the transition state occurs in the centre of the contact at an asperity force $F_{i}=F_{i_{c r i t}}$. In the presence of the full contact state the numerical calculations are now performed separately for the two regions and summed up based on the respective area ratio. The force acting on the asperity inside the transition zone equals $F_{i}=\bar{p}_{\text {trans }} * \lambda^{2}$ and outside $F_{i}=\bar{p}_{\text {asp }} * \lambda^{2}$ as given by equations 3.11 and 3.12 . 


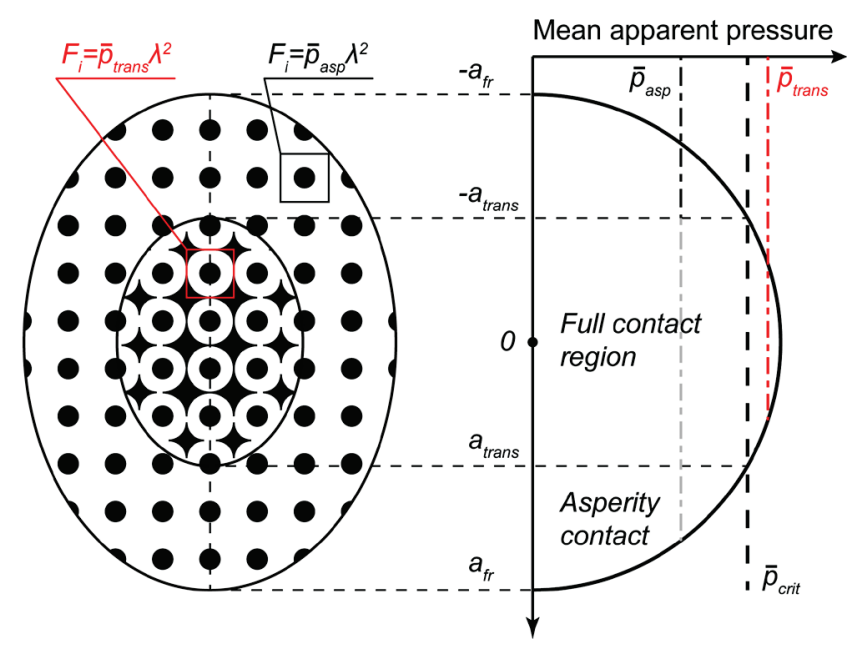

FIGURE 3.3 Schematic representation of the contact states, ridge mean pressures and asperity forces.

\subsection{EXPERIMENTAL EVALUATION OF THE SKIN ELASTIC}

\section{MODULUS}

Calculation of the finger pad contact area requires two finger specific parameters, i.e., fingerprint ridge contact area and elastic modulus of the skin, both of which are subjected to change with time and environmental conditions. The contact area of a finger pad can be measured with an ink imprint, a fingerprint scanner or estimated with an analytical model based on the finger pad and papillary ridge dimensions, while evaluation of the skin stiffness requires dedicated micro-indentation measurements. By using the contact transition as an indication for the skin deformation, the skin elastic modulus was estimated empirically in Paper A based on the performed friction measurements.

Contact transition was observed through the finger pad sliding measurements, which represented in a considerable increase of friction due to the predominantly adhesive contact. The critical normal load from 
the experimental results, at which the contact transition occurred, was converted to asperity forces based on Eq. 3.5. Ridge contact area with the samples was estimated based on the fingerprint scanner measurements. Given that the material elastic modulus is known, and the skin microdisplacement equals the texture asperity height, skin elasticity was estimated to be in the order between $0.2 \mathrm{MPa}$ and $0.5 \mathrm{MPa}$. The calculations were performed for four various silicone rubber grades and are given in Table 3.1.

TABLE 3.1 Calculated Young's modulus of the skin and micro-deformations at the point of contact transition for the four tested materials.

\begin{tabular}{|c|c|c|c|c|}
\hline $\begin{array}{l}\text { Silicone rubber grade } \\
\text { (Smooth-on, USA) }\end{array}$ & $\begin{array}{l}\text { Mold Max } \\
10\end{array}$ & $\begin{array}{l}\text { Mold Max } \\
\qquad 30\end{array}$ & $\begin{array}{l}\text { Mold Max } \\
\qquad 40\end{array}$ & $\begin{array}{l}\text { Mold Max } \\
\quad 60\end{array}$ \\
\hline $\begin{array}{l}\text { Sample material elastic } \\
\text { modulus (MPa) }\end{array}$ & 0.2 & 0.75 & 1.3 & 2.3 \\
\hline Skin deformation $(\mu \mathrm{m})$ & 8.7 & 22.9 & 25.4 & 29.1 \\
\hline Asperity deformation $(\mu \mathrm{m})$ & 21.2 & 6.1 & 4.8 & 2.5 \\
\hline $\begin{array}{l}\text { Estimated skin Young's } \\
\text { modulus (MPa) }\end{array}$ & 0.5 & 0.2 & 0.25 & 0.2 \\
\hline
\end{tabular}




\subsection{RELATION TO SURFACE TEXTURE DIMENSIONS}

Based on the model input of the person specific finger ridge contact area, skin elastic modulus and normal load, a relation of contact area to the texture parameters was analysed. A parametric sweep simulation was performed to calculate the real contact area fraction as a function of the surface texture dimensions. The texture of the surface topography was simulated as a square packed hemispherical asperity design with a feature radius equal to its height. The resultant graph for the normal load of $1 \mathrm{~N}$ and a surface with the elastic modulus of $1 \mathrm{GPa}$ is shown in Figure 3.4 .

The linear relationship observed from the figure can be explained by the dimensionless texture coefficient $k_{t}$ obtained from the Hertzian model in Eq. 3.6:

$$
k_{t}=\frac{\sqrt{h_{a s p}^{3} R_{a s p}}}{\lambda^{2}}
$$

For the simulated texture design, where $h_{a s p}=R_{a s p}$ the dimensionless texture parameter can be simplified to $R_{a s p} / \lambda$. The upper part of the graph characterises the asperity contact state. Contact area can be reduced either by decreasing the feature radius or by increasing their relative spacing. The boundary for the contact state change is defined by the radius to spacing ratio $R_{\text {asp }} / \lambda=0.18$ and transition to full contact is reached for this texture around 0.10 , which corresponds to about $10 \%$ and $3 \%$ of the textured surface area, respectively. The lower limit of the model is expected to be formed by the dimensions of a single corneocyte cell, which corresponds to the asperity spacing around $50 \mu \mathrm{m}$. At lower feature sizes the roughness of the skin has to be taken into account, which falls outside the scope of this thesis. 


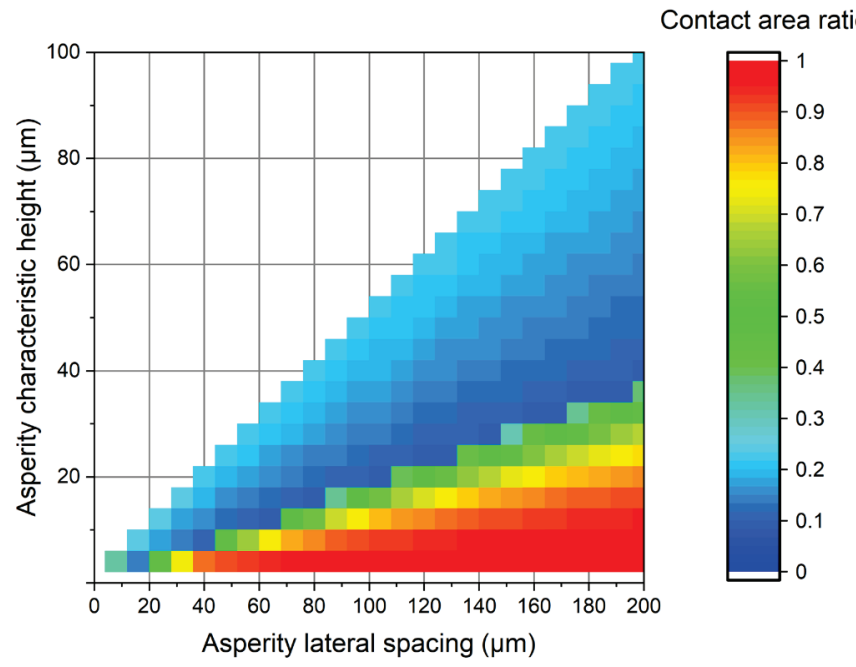

FIGURE 3.4 Real contact area ratio as a function of texture dimensions at $1 \mathrm{~N}$ normal load and a surface with the elastic modulus of $1 \mathrm{GPa}$.

\subsection{FINITE ELEMENT METHOD SIMULATIONS}

Finite element method (FEM) analysis was applied to study the contact behaviour of a single texture feature under simultaneous tangential and normal loading. In Paper B a parametric study was performed to investigate the directional frictional behaviour of ellipsoidal bump textures and the influence of the texture feature size, Young's modulus and sliding direction on the contact area development.

Each asperity model was represented by two bodies: an asperity and a sliding block, both modelled as isotropic linear elastic elements (Figure 3.5). The latter is important to notice as it makes it different from simulations with rigid texture feature such as, e.g., in the work of LeyvaMendivil et al. [102]. Bodies were meshed with elements of $10 \mu \mathrm{m}$ and distortion control was used to prevent excessive mesh warping. Interaction between two surfaces was defined by penalty friction 
formulation. To represent an adhesive friction component, which remains predominant in studied contact conditions, the friction coefficient was set to 1 , while the maximum shear stress was limited to $10 \mathrm{kPa}$. Therefore, the interfacial shear force remained mostly independent of contact pressure, simulating an adhesive contact. Surface self-contact was set to frictionless to reduce simulation times.

Initial boundary conditions were specified to restrict body movement. Asperity block was fixed at the bottom plane and its sides were not allowed to move in XZ plane. Each finite element method analysis consisted of two calculation steps with prescribed displacement boundary conditions:

- Vertical translation to induce normal load;

- Horizontal translation at constant normal displacement which ensured contact sliding.

There was no intention to simulate the mechanical behaviour of the skin with the current FEM study; yet only to represent a similar normal

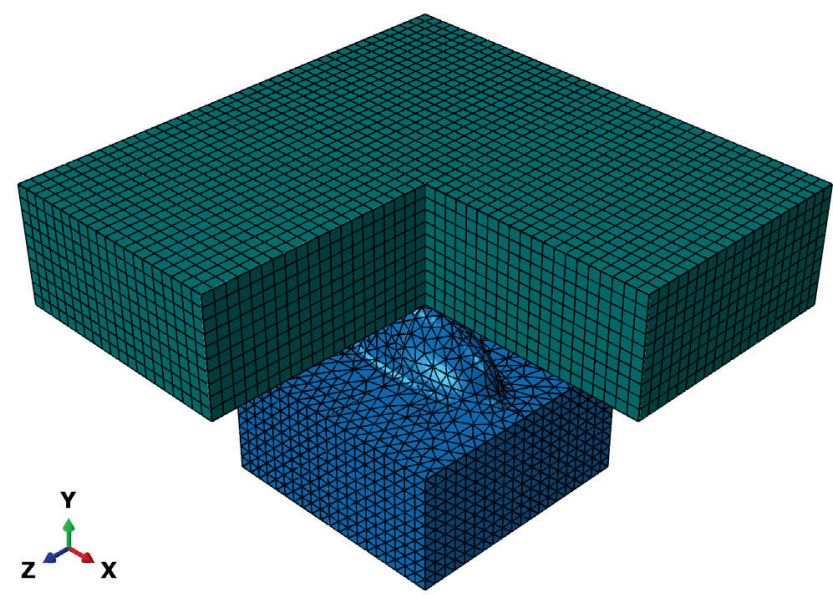

FIGURE 3.5 FEM system layout and body meshing for shear deformation analysis. Upper block is shown under a section view. 
displacement. Therefore, the upper body was assumed as an elastic body with a Young's modulus of $0.4 \mathrm{MPa}$, which was estimated Paper A. Asperity properties were set according to the tested material with the elastic modulus of 0.2 MPa. Poisson's ratio of 0.49 was used for both bodies [48,103]. For a continuous sliding inertial forces do not alter solution significantly. Therefore, fixed mass scaling was applied to the entire model to reduce calculation times.

\subsection{CONCLUSIONS}

A contact model, capable of predicting the contact transition effect in tactile friction was developed based on a combination of analytical and numerical methods. The boundary element method is applied to calculate the contact area on the micro-scale for the custom feature topography, while the simulation parameters are obtained from the analytical solution and finger pad pressure distribution on the mesoscale.

Effective elastic modulus of the skin, applicable on the microscale, was estimated empirically by observation of the contact transition during tactile friction and using it to quantify local skin deformation. The Young's modulus was estimated to be in the range between $0.2 \mathrm{MPa}$ and $0.5 \mathrm{MPa}$, which is slightly higher, but of the same order as values calculated from fingerprint ridge deformation models.

Conditions for the contact transition were found to depend on texture parameters and were expressed for a spherical bump surface as a dimensionless coefficient $k_{t}=\sqrt{h_{\text {asp }}^{3} R_{\text {asp }}} / \lambda^{2}$. For materials with stiffness higher than that of skin the transition to full contact state is predicted to occur for textures with $k_{t}<0.03$ at $1 \mathrm{~N}$ normal load. For the hemispherical asperities, the boundary condition can be also approximated by a radius to spacing ratio $R_{\text {asp }} / \lambda<0.18$ or textured surface area ratio below $10 \%$. 


\section{TACTILE FRICTION MEASUREMENTS}

\subsection{EXPERIMENTAL}

\subsubsection{Test setup}

The numerical models were validated by experimental work performed in-vivo, using a dedicated setup designed by Klaassen et al. [66]. The test rig was adapted for the finger pad friction measurements and consists of an enclosed chamber with a linear reciprocating tribometer (Figure 4.1). A linear stage is driven with a lead screw and carries a balancing lever with a loadcell on one end and a counterweight on the other. The right hand is placed on a stage with an index finger inclination angle of $30^{\circ}$ relative to the sample. The sample is moved from a subject's body with a constant velocity of $2 \mathrm{~mm} / \mathrm{s}$ and a total sliding distance of $20 \mathrm{~mm}$. After each stroke, the sample is lifted, and the stage is returned to its starting position.

The fixed position of the finger during the friction measurements helped to reduce the deviations of the normal load and friction force. A typical example of the friction measurements is provided in Figure 4.2. A dynamic coefficient of friction, which characterises a stable relative sliding motion of the bodies, is extracted from the measurement data and 
a

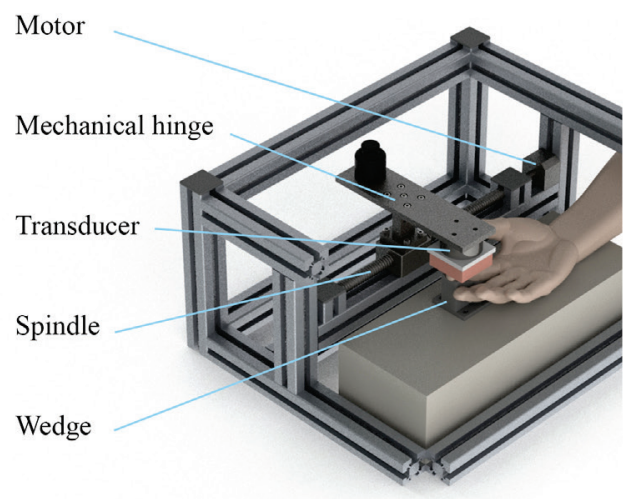

FIGURE 4.1 Schematic representation of the test setup used for the friction measurements (a) and a top-down view on its inner components (b).

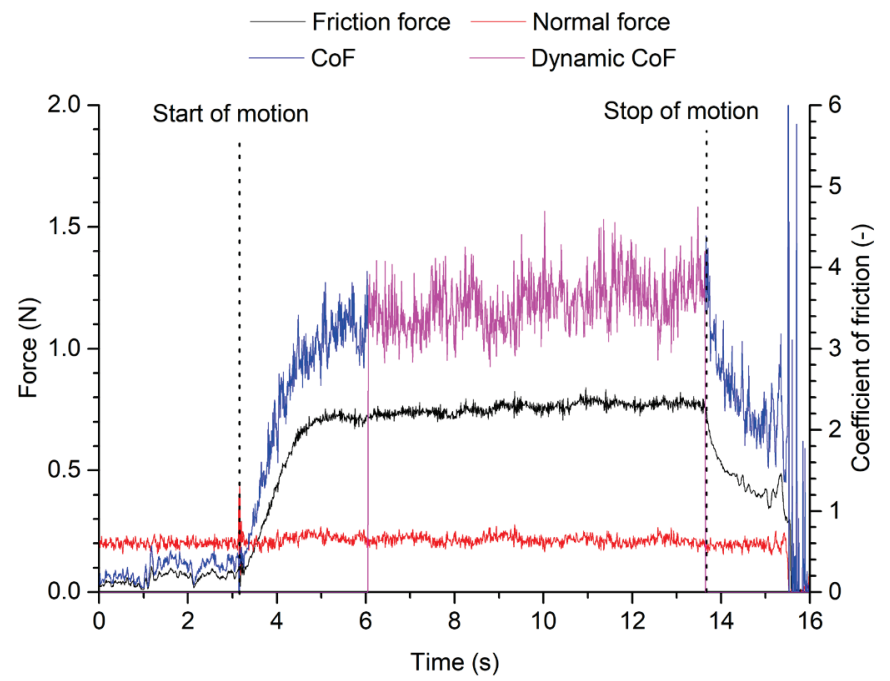

FIGURE 4.2 Typical force signals with calculated and extracted dynamic friction coefficient 
used for evaluation. The tests were performed with a range of normal loads up to $5 \mathrm{~N}$ to observe the change of the friction behaviour with applied force. The normal load was increased during the measurement series to reduce the variation of the contact area due to the visco-elastic behaviour of the finger pad.

\subsubsection{Textured samples}

Textured surfaces were based on square-packed bump designs, characterised by evenly spaced rounded asperities with a flat base surface. This feature layout was selected for multiple reasons: rounded features reduce the deformation friction, tooling for the forming process can be accurately produced by the laser surface texturing and feature periodicity in both lateral directions can be conveniently included in numerical calculations. The designs are defined by the features' radii $R_{a s p}$, their height $h_{\text {asp }}$ and a lateral distance $\lambda$ between them.

Negative master moulds were produced on stainless steel sheets by direct laser texturing using a picosecond laser (Edgewave, Germany) with a pulse

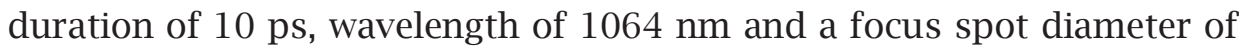
$10 \mu \mathrm{m}$ [104]. Samples were formed from silicone rubber by direct moulding into a steel master or into a master mould replicated in polyurethane, which reduced adhesion during demoulding process.

\subsection{INFLUENCE OF THE CONTACT STATES}

Two textured surface designs were selected to evaluate the three contact states through tactile friction measurements, validate the proposed contact model and estimate the skin elastic modulus based on deformation. The textured and reference samples were produced in four various rubber grades with elastic moduli of $0.2,0.75,1.3$ and $2.3 \mathrm{MPa}$. 

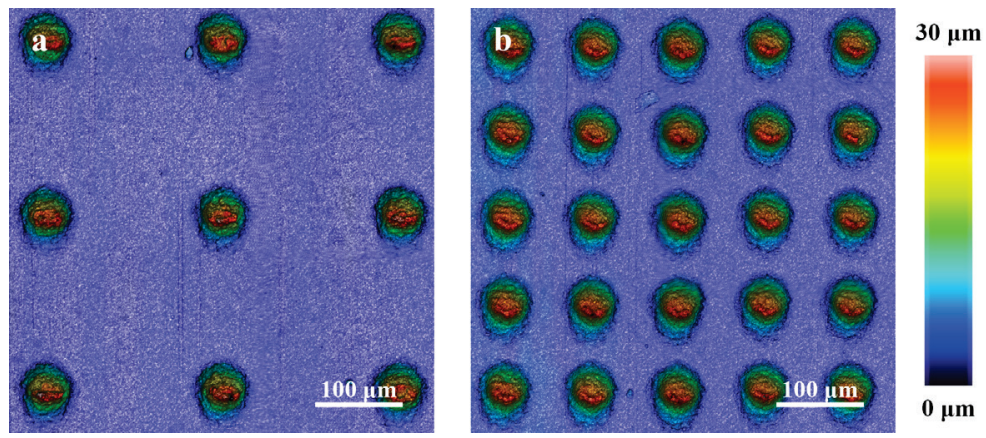

FIGURE 4.3 Confocal images of the produced sample textures with asperity spacing of 200 $\mu \mathrm{m}(\mathrm{a})$ and $100 \mu \mathrm{m}(\mathrm{b})$.

Both textures had an average asperity tip radius of $19 \mu \mathrm{m}$ and a height of $28 \mu \mathrm{m}$. The patterns differed in the asperity density introduced by the various feature lateral spacings of 100 and $200 \mu \mathrm{m}$, referred in the text as L100 and L200, respectively (Figure 4.3).

For the smooth reference surfaces, the coefficient of friction reduced with an increase of the normal load and followed a commonly observed power law function (see Chapter 2): $\mu \propto F^{n-1}$, with $n$ being equal to $0.75,0.67$, 0.58 and 0.79 for all the rubber grades from the softest to the hardest, respectively. All L200 textures showed a completely different trend. At low normal loads, the coefficient of friction was significantly reduced, but it rose quickly with a further increase of normal load, reaching its maximum around $0.8 \mathrm{~N}$ load. This peak is followed by a steady decline with the values tending to the friction results of the non-textured (reference) samples. The high density L100 texture showed significantly lower values of the friction coefficient, which remained almost constant for the entire range of the tested normal loads. Only the softest L100 sample demonstrated a linear increase in the coefficient of friction, which was connected to the predominant deformation of the texture asperities and higher hysteresis friction losses due to the larger material displacement [72]. 
Friction measurements were used to evaluate the contact model prediction. Contact area calculated with the proposed numerical method was fitted to the empirical results based on the equations 2.2 and 2.4:

$$
\mu=\mu_{\text {adh }}+\mu_{\text {def }}=\frac{\tau_{0} A_{r e}}{F}+\alpha+\beta\left(\frac{9}{128 R_{f p}}\right)^{\frac{2}{3}}\left(\frac{F}{E_{0}^{*}}\right)^{\frac{1}{3}}
$$

The solution was restricted by using constant values for the intrinsic interfacial shear strength $\tau_{0}$ and the pressure coefficient $\alpha$, while the hysteresis coefficient $\beta$ varied with the sample stiffness. The quality of the resultant fit to friction measurements is shown in Figure 4.4. The

contact state difference explains the significant variation in the observed friction values and trends between samples. The L100 textures remained in the asperity contact with the skin through the whole range of the normal loads. The L200 textures passed a contact transition and reached the full contact state with the total contact area comparable to that of the reference samples.

\subsection{INFLUENCE OF ASPERITY DEFORMATION}

By introducing an asymmetry to a surface texture design, the tactile frictional behaviour can be changed with respect to the sliding direction. This is generally achieved through the creation of sinusoidal or parallelridge textures $[81,83,105]$, which utilize skin hysteresis either to reduce the contact area during sliding or to promote deformation. To investigate whether a bidirectional frictional behaviour can be created with a microscale surface texture, four textures with ellipsoidal asperities were designed. Micro-texture dimensions imply that the difference in friction is achieved not by the deformation of the finger pad skin, but by changing the contact area. 
a

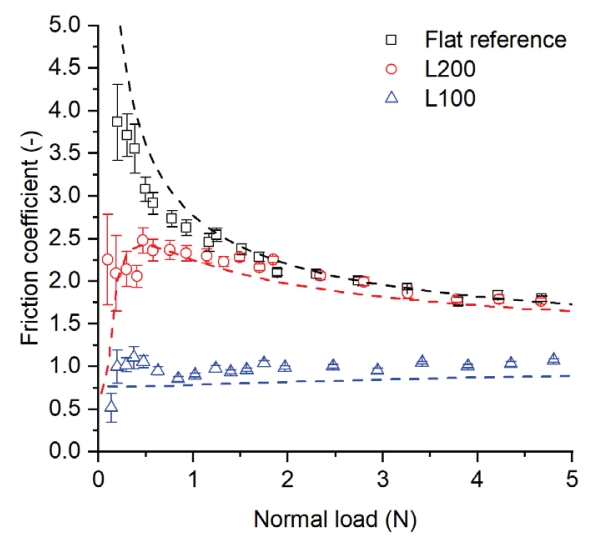

C

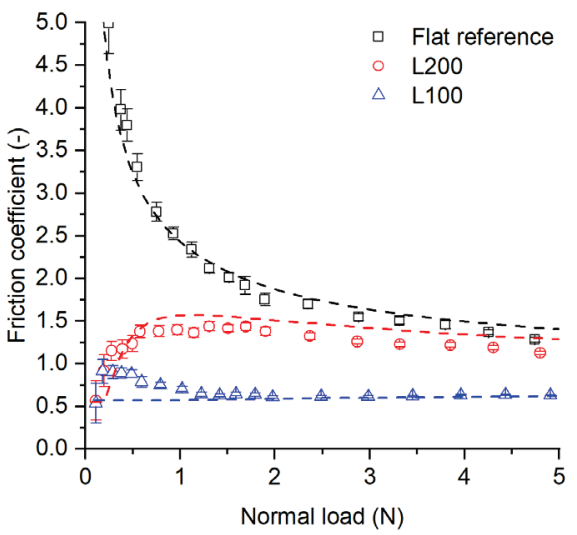

b

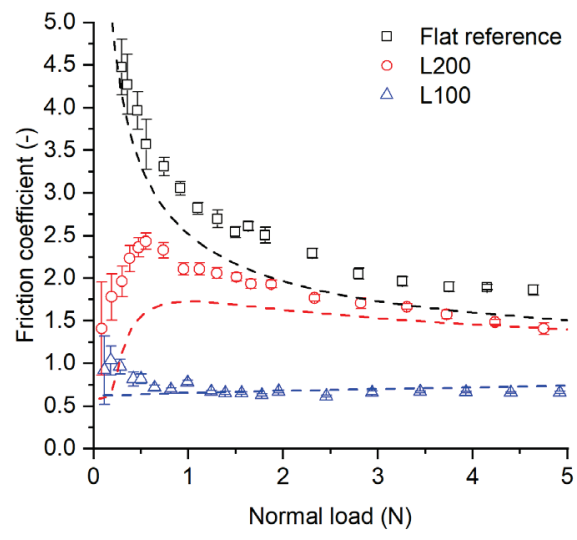

d

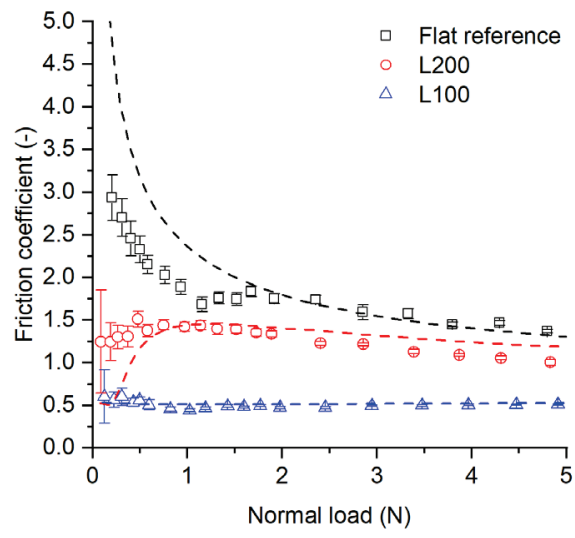

FIGURE 4.4 Friction measurements (points) and the closest model fit (dashed lines) for the friction coefficient measurements. Results are grouped by the silicone rubber grades: $0.2 \mathrm{MPa}$ (a), 0.75 MPa (b), 1.3 MPa (c), 2.3 MPa (d). 
Bump features with elongated ellipsoidal asperities with a lateral spacing of $200 \mu \mathrm{m}$ were used for the study. The asperities had the same width of $50 \mu \mathrm{m}$ and two major lengths of 100 and $150 \mu \mathrm{m}$ (Figure 4.5). Each of those designs were produced with the heights of 30 and $50 \mu \mathrm{m}$ from silicone rubber. A rubber grade with an elastic modulus of $0.2 \mathrm{MPa}$ was selected to promote the deformation of elastomer features over the skin surface. Test measurements were performed parallel and perpendicular to asperity length dimension and were repeated twice for each sample set to confirm that the obtained results are related to the texture design and independent of skin properties.

Textures with a height of $30 \mu \mathrm{m}$ resulted in lower friction coefficients in sliding contact with the finger pad than the reference samples. However, the directional sliding difference became evident only with increase in the asperity heights to $50 \mu \mathrm{m}$. Sliding perpendicular to the asperity length increased the friction force by a factor of 2 at normal loads below $0.5 \mathrm{~N}$ (Figure 4.6). With increase in the applied load the textures reached the full contact state and the spread in friction coefficient reduced. From this it is concluded that the directional sliding behaviour was caused by the contact area differences and has a predominantly adhesive nature.

The difference between parallel and perpendicular sliding directions was analysed with finite element simulations. A series of calculations was performed for each ellipsoidal asperity size. Normal displacement was changed incrementally from 5 to $40 \mu \mathrm{m}$ with a step of $5 \mu \mathrm{m}$. For elliptic structures with the height of $30 \mu \mathrm{m}$, a change of sliding direction did not affect the results; both contact area and normal loads remained comparable. However, for the asperities of $50 \mu \mathrm{m}$ height, the change in the deformation with the sliding direction became evident. Increased asperity height under interfacial shear forces promoted an elastic 

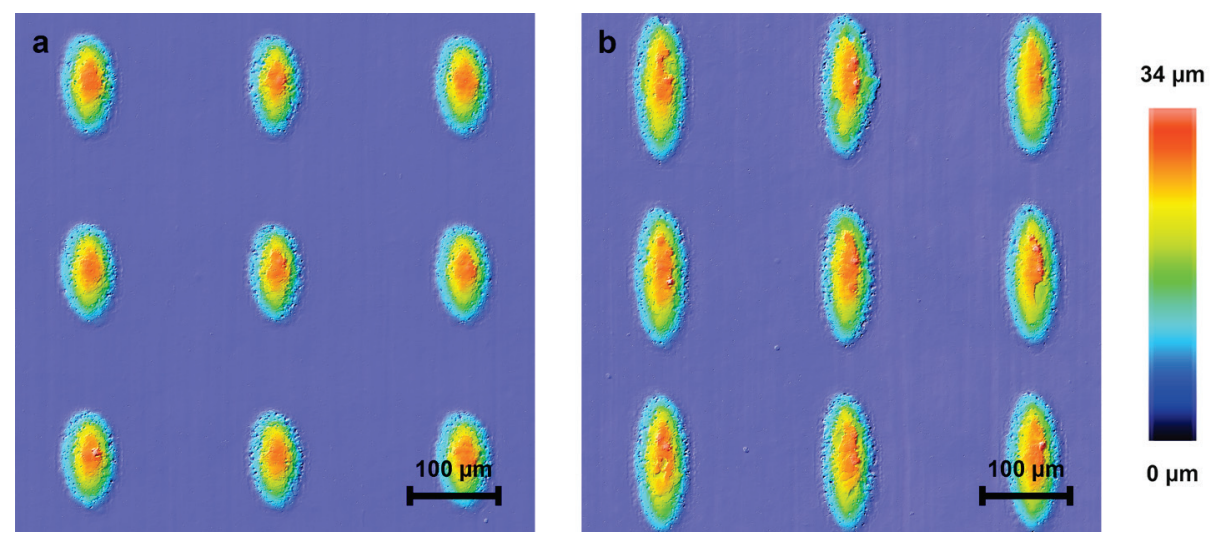

FIGURE 4.5 Ellipsoidal textures with the asperity lengths of 100 (a) and $150 \mu \mathrm{m}$ (b).
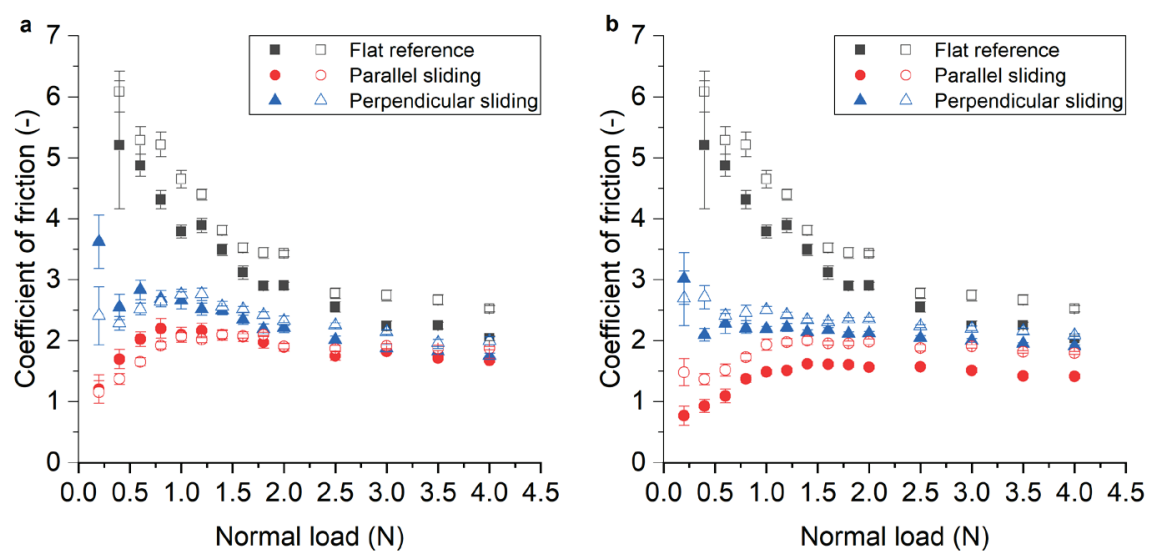

FIGURE 4.6 Coefficient of friction for $50 \mu \mathrm{m}$ height textures with asperity lengths of 100 $\mu \mathrm{m}$ (a) and $150 \mu \mathrm{m}$ (b). Marker filling denotes two series of measurements. 
deflection or asperity "bending" during perpendicular sliding, compared to usual "barrelling" deformation.

The difference in sliding direction can be seen in Figure 4.7, which compares typical normal load and contact area curves during simulation. For the parallel sliding case there is a slight increase of the normal load at the start of the tangential translation step. However, it gradually returns to the initial value. Its calculated contact area also remains stable. Change of the sliding direction and extensive asperity deformation causes the normal load to drop from 0.33 to $0.19 \mathrm{mN}$, where it stabilizes for the rest of the motion. It also leads to minor fluctuations and a gradual increase of the contact area.

The data of the stable region during tangential sliding was averaged for each simulation result to represent the contact area as a function of normal load and is shown in Figure 4.8. At low normal loads there is no noticeable directional difference in the contact area. However, with increase in the shear force with the contact area the asperity starts to bend. It leads to substantially higher contact areas with respect to the
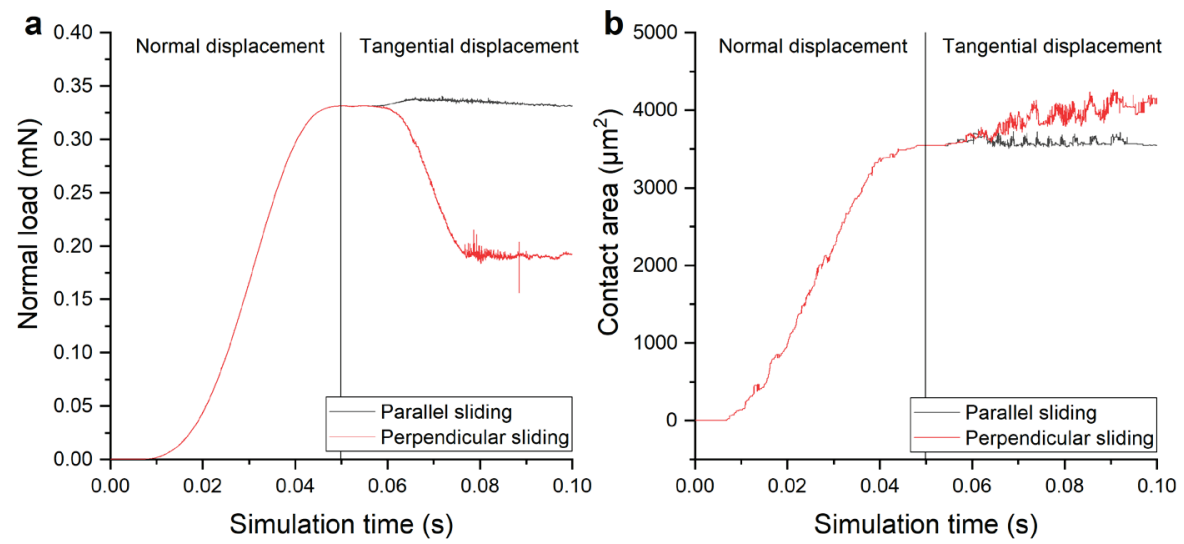

FIGURE 4.7 A typical normal load (a) and contact area (b) during numerical simulation. 
normal load. For longer asperities (150 цm), the contact area nearly doubles at the same normal load as shown in Figure 4.8b.

Effectively, the asperities in the deformed state can be considered as a different texture with a lower asperity height and larger equivalent radius. Therefore, it results not only in a higher friction at asperity contact state, but the transition to the full contact state also starts at lower normal loads. As expected, an asperity with a larger equivalent radius can support a higher normal load before the contact transition starts.

Computational results were in a good agreement with experimental measurements. Asperity deflection might well explain the friction gain with the change of the sliding direction. The estimated increase in the contact area for the textures approaches the factors of 1.5 and 2, which matches the relationship between friction coefficients for these textures. Furthermore, the maximum difference was reached at asperity contact state before the transition to full contact, which corresponds to the low normal load region in the friction graphs.
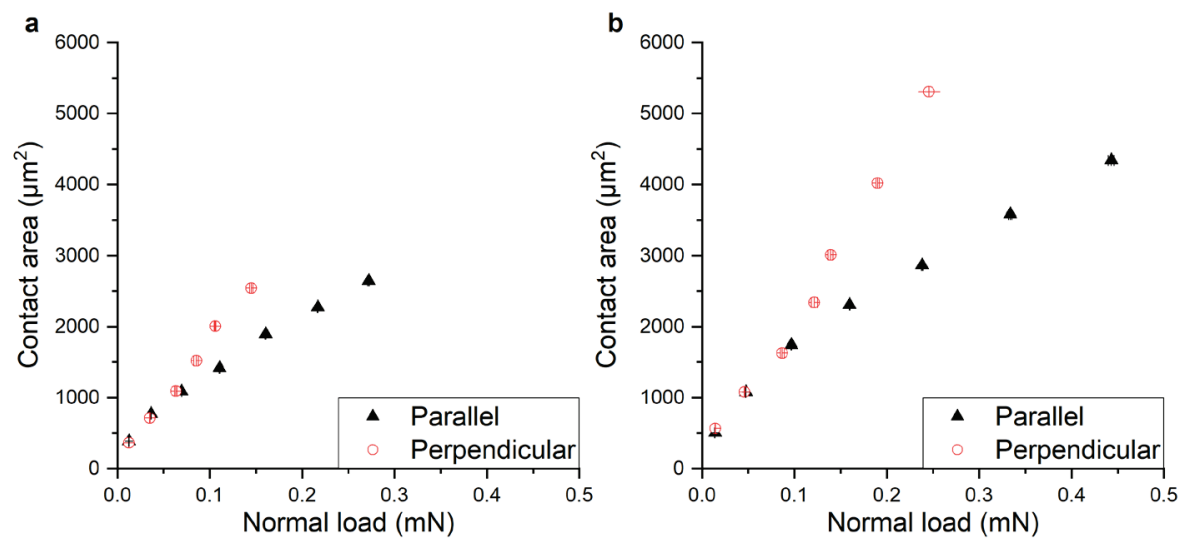

FIGURE 4.8 Simulated contact area as a function of normal load for the features with the lengths of $100 \mu \mathrm{m}(\mathrm{a})$ and $150 \mu \mathrm{m}$ (b). 
The highest difference between sliding directions is predicted during asperity contact and before the transition to the full contact state happens. These results show the feasibility of the asymmetric texture designs for tactile friction, which are based on the change in the contact area and not the deformation of the skin. The directionality effect increases with asperity major radius and height. FEM simulations show a significant influence of material elastic modulus on the normal load at which the asperity deformation starts. In the absence of asperity deformation, friction differences due to sliding direction are expected to depend on skin properties, such as its tangential stiffness and viscoelastic response.

\subsection{FRICTION NORMALISATION}

The coefficient of friction in tactile sliding is rarely compared between volunteers due to its variation based on personal and environmental differences [66,106]. A possible approach to compare tactile friction quantitatively, is to normalise with respect to the influence of the personal finger pad parameters. To evaluate this, three textured surfaces were selected similar to the samples used previously in the thesis (Figure 4.9).

Texture designs were chosen with the purpose of representing three distinctive contact states, i.e., contact with asperity features (S100), full contact with a texture valley (S200) and a gradual transition between the two (E200). Therefore, the ellipsoidal texture was tested sliding parallel to the asperity lengths. Features for the S100 and S200 surfaces had the same tip radius of $27 \mu \mathrm{m}$ and height of $22 \mu \mathrm{m}$, while the E200 samples had an ellipsoid width, length, and height of $50 \mu \mathrm{m}, 100 \mu \mathrm{m}$ and $47 \mu \mathrm{m}$, respectively. A flat non-textured sample with an average roughness of 70 $\mathrm{nm}$ was used for the reference measurements. The measurements were 

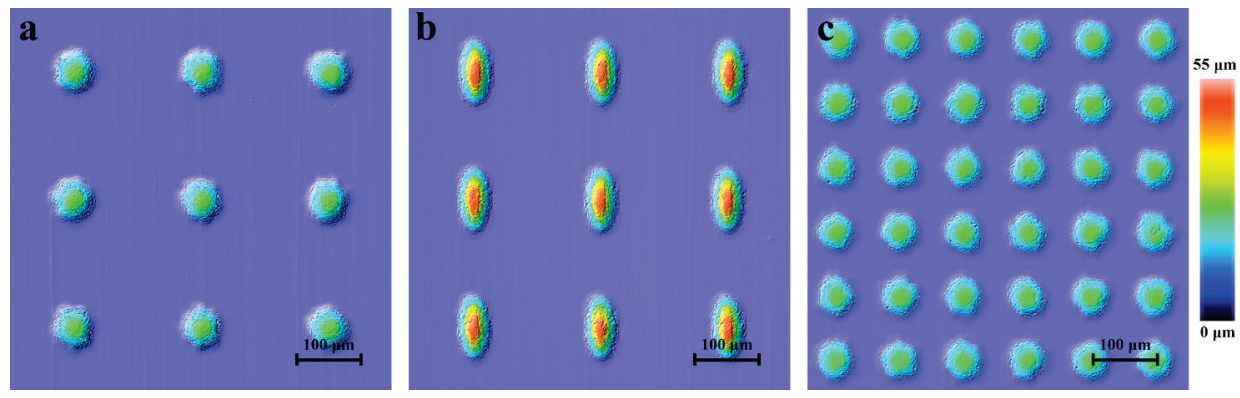

FIGURE 4.9 Surface topography of S200 (a), E200 (b) and S100 (c) obtained with confocal microscope.

performed with a test group of 10 volunteers to ensure the variation in individual finger parameters. The sample test order was randomised each time, and the measurements for a person were performed within the same day.

As anticipated, the calculated friction coefficients varied greatly between volunteers. The difference between the highest and the lowest values for the equivalent samples and normal loads reached a factor of 4 . Flat reference samples showed a reduction of friction with increase of the applied normal load, reaching the range of $0.9-2.5$ at $4 \mathrm{~N}$ (Figure 4.10a). Textured samples S200 and E200 exhibited significantly lower coefficient of friction at low normal loads as observed on Figure 4.10b and Figure 4.10c. However, friction increased with the applied normal loads and stabilised in value ranges of $1.1-2.0$ and $0.7-1.3$ for S200 and E200 respectively. The lowest friction coefficient was observed for the highest density texture S100 and remained almost independent of the normal load at respective values of 0.35 - 1.0 (Figure 4.10d). 

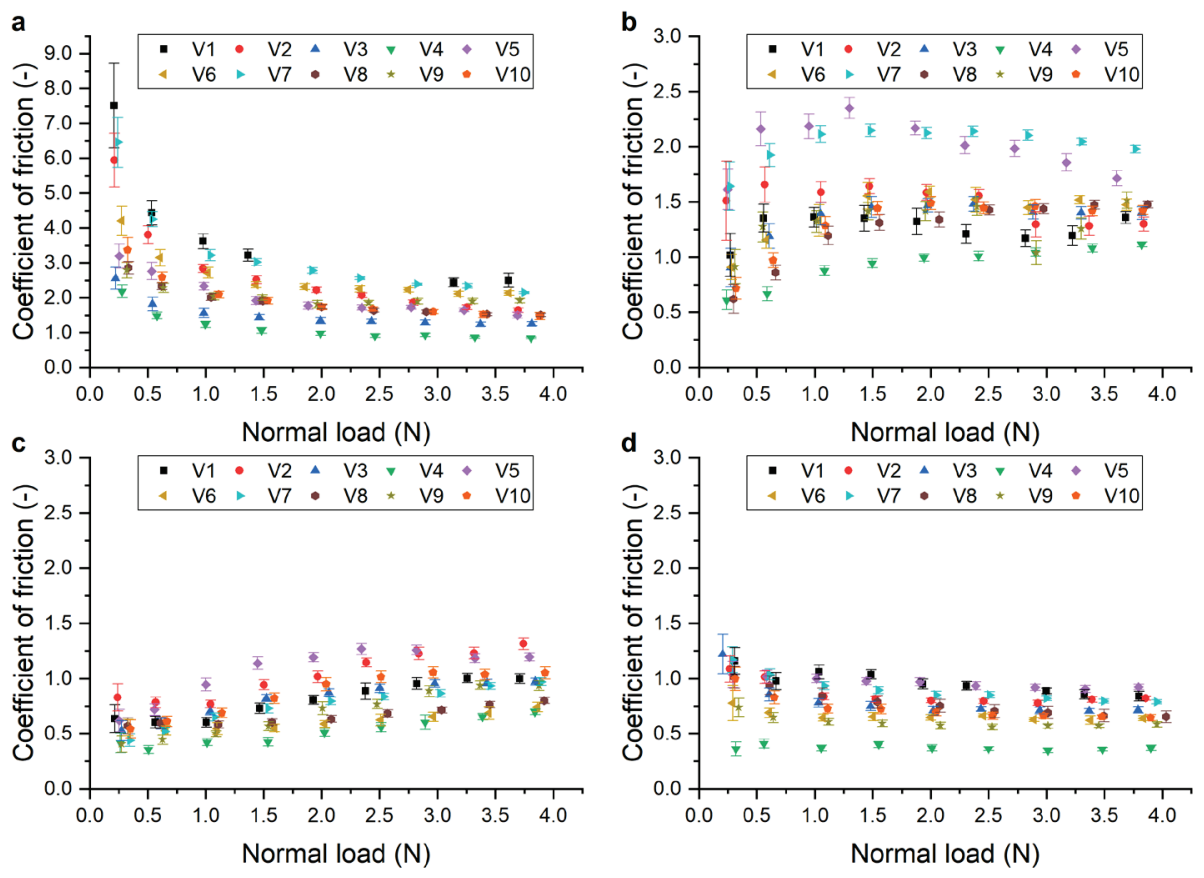

FIGURE 4.10 Calculated coefficient of friction for all the subjects grouped by surface texture: reference (a), S200 (b), E200 (c), S100 (d). Note the difference in Y-scale values for graph (a).

Normalisation of the friction measurements for each volunteer was performed with the following assumptions:

1. Fingerprint ridge contact area $A_{f r}$ as a function of normal load does not change for a person during the whole measurement series.

2. The real contact area with the reference sample $A_{r e_{r e f}}$ approaches the ridge contact area of the finger pad $A_{r e_{r e f}} \rightarrow A_{f r}$ at all normal loads. It implies that the roughness of the skin on the fingerprint ridges is considered negligible. 
3. The deformation component of friction is small and, therefore, the friction force is based purely on adhesion, so that $F_{f}=F_{a d h}=$ $\tau A_{r e}$, where $\tau$ is the interfacial shear strength and $A_{r e}$ is the real contact area.

The friction data for the surface of interest can be represented as a unitless real contact area fraction. Considering that the interfacial shear strength $\tau$ remains constant for the dataset, the contact area ratio $\psi$ can be found as a quotient of the friction coefficients or the friction forces:

$$
\psi(F)=\frac{\mu_{t e x}(F)}{\mu_{r e f}(F)}=\frac{\tau A_{r e}(F)}{\tau A_{f r}(F)}=\frac{F_{f_{t e x}}(F)}{F_{f_{r e f}}(F)}
$$

where the $F_{f_{t e x}}(F)$ is the measured friction force for the textured sample and $F_{f_{r e f}}(F)$ is a power fit function for the flat reference. Plotting the contact ratio against the normal load allows to evaluate contact area development and compare it between datasets.

However, with a variation of ridge contact area between individuals, the same normal load can correspond to a different ridge pressure. Such variance can be simplified by plotting the data as a function of the mean ridge pressure and effectively comparing the contact area development for the micro- length scale. Mean ridge pressure cannot be found numerically without additional measurements, but it can be substituted by a ratio of the normal and tangential forces. Given that the mean ridge pressure is a function of the normal load as $\bar{p}(F)=F / A_{f r}(F)$, friction data from a reference surface can be used to obtain a dimensionless force ratio:

$$
\bar{p}(F) / \tau=F / \tau A_{f r}(F)=F / F_{f_{\text {ref }}}(F)
$$


By plotting the contact area ratio as a function of the force ratio $\psi(\bar{p} / \tau)$, a power function component can be reduced, which allows comparison of the micro-contact development between different persons. For the linear elastic case, the deformation is linearly proportional to the applied stress. It implies that for the same textured surface the function of $\psi(\bar{p} / \tau)$ is expected to vary between individuals linearly with the interfacial shear strength $\tau$ and effective Young's modulus; both can be considered unique and constant for each volunteer dataset.

After normalising friction by the reference values for each volunteer, the results as summarised in Figure 4.11 were constructed. Interestingly, the difference between personal measurements appears to have a linear relationship. The change of the contact state for the texture E200 shown on Figure $4.11 \mathrm{~b}$ became evident for all volunteers. It consists of two parts: initial asperity contact slope comparable to the results of S100 in Figure 4.11c and subsequent change of inclination similar to S200 in Figure 4.11a - full contact state. On the contrary, the surface S100 has a linear trend for all participants. Normalised data for the S200 show the largest variation due to the immediate transition to the full contact state. For example, volunteers V3, V4 and V5 show a calculated real contact ratio above 1 (Figure 4.11a), which contradicts the second initial assumption. 

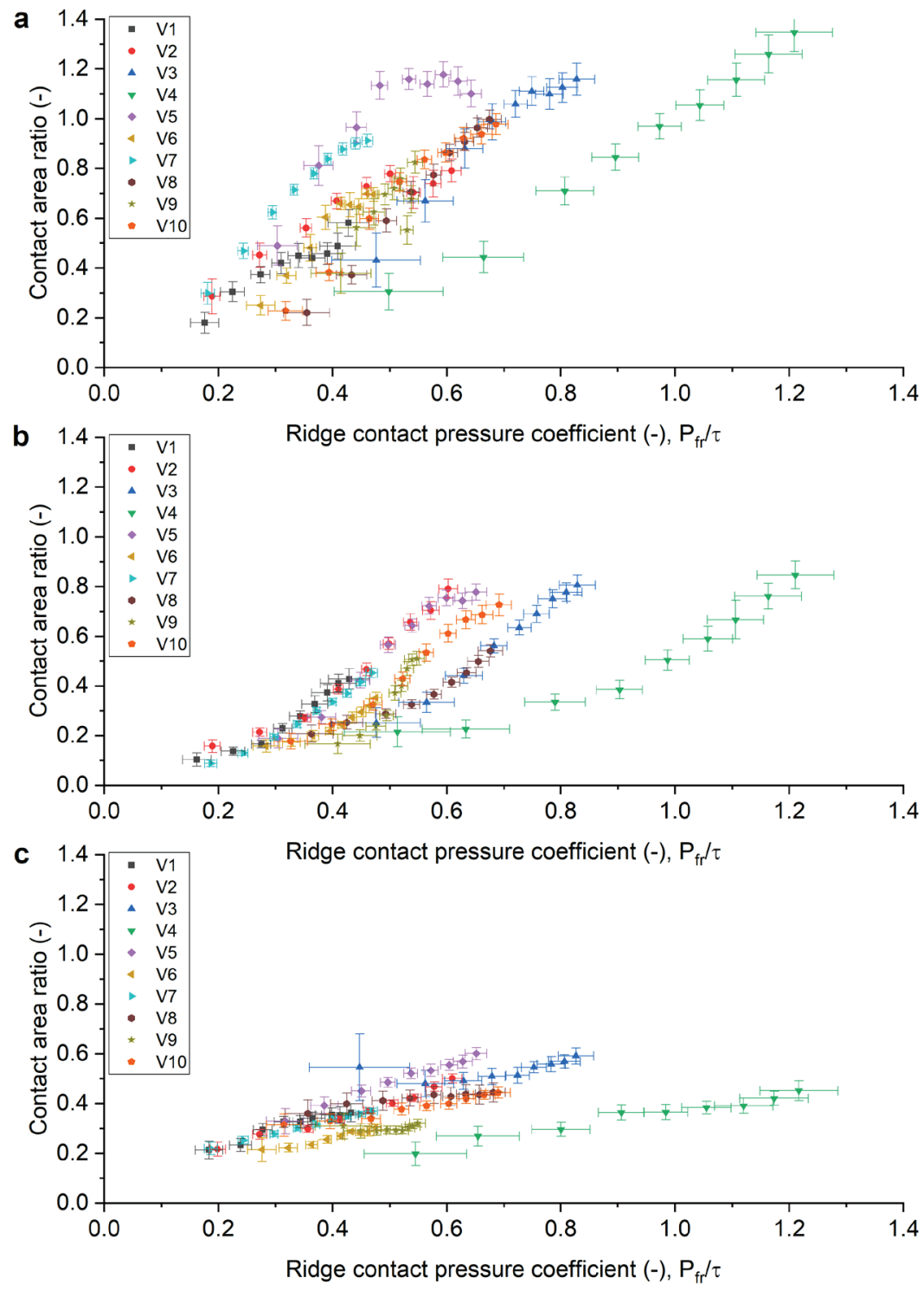

FIGURE 4.11 Calculated contact area ratio as a function of pressure ratio grouped by texture: S200 (a), E200 (b), S100 (c). 
The validity of using a reference sample for normalisation can be further analysed by examining the results for the textured surfaces E200 and S100 more closely. E200 showed the lowest coefficient of friction for all participants at normal loads below 1 N. However, at higher loads, friction increased due to the transition to full contact state. The trends for the two textured surfaces E200 and S100 can be compared to estimate a point of intersection. At this point the friction coefficients and, therefore, contact area ratios become equal for two different textures. The intersection point corresponds to particular skin deformations as defined by the specific surface topographies.

This point of intersection can be clearly defined for each volunteer from the friction results, as well as calculated with a contact model. It allows application of the same normalisation approach to both numerical and empirical methods for comparison. Dividing the pressure coefficient axis by the particular value of intersection allows elimination of the remaining personal differences from the graphs in Figure 4.11. More details on this calculation step are given in Paper C. The convergence of the data for the textured surfaces L200 and S100 means that deformation on the asperity scale remains proportional to pressure for all volunteers. Notably, higher outlying values correspond to participants V3, V5 and V8, who showed reduced friction against the reference samples introducing error to the normalised results.

After the normalisation of pressure, the total deformation range can be compared to experimental results in Figure 4.12. The modelled results are fitted to the frictional data as $\psi(\hat{p})=\psi_{s}(\hat{p})+0.2 \hat{p}$, where $\psi_{s}(\hat{p})$ is a contact area ratio function obtained from the numerical model and $\hat{p}$ is a normalised mean ridge contact pressure. Interestingly, the linear fit does not change with texture or participant. It suggests that coefficient of friction is proportional to pressure. Furthermore, the volunteer data lies within a small range of normalised ridge pressure values. The participants 

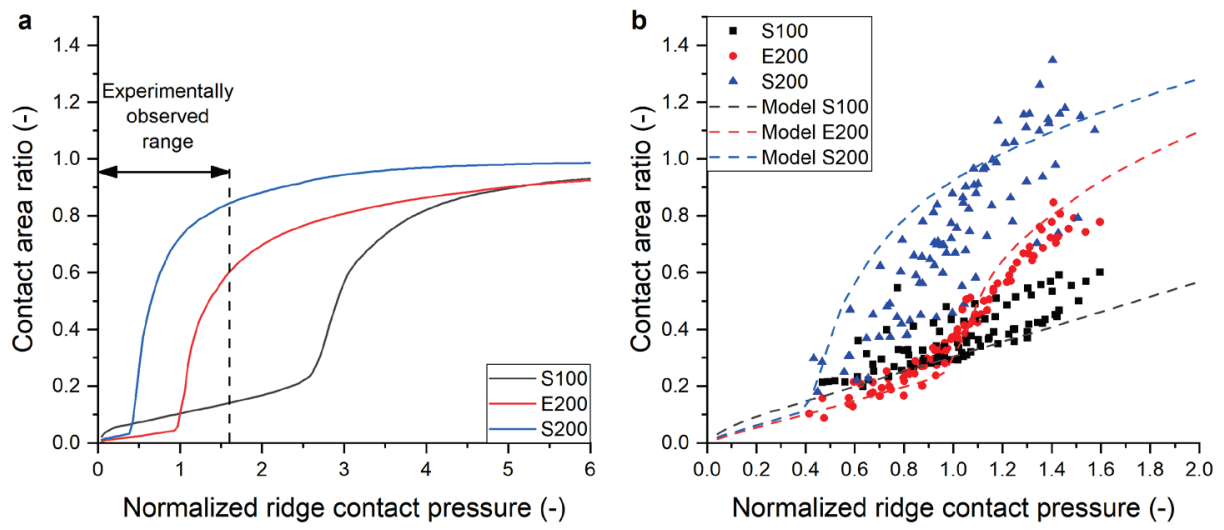

FIGURE 4.12 Contact model results for textured surfaces (a) and its fit to the normalised friction data (b).

experienced a similar range of skin micro-deformations, even though the personal differences were prominent. For this to hold true, the higher skin elastic modulus must be accompanied by a corresponding increase in ridge pressure and vice versa, which can be achieved by the deformation of fingerprint ridges. This could explain why perception of surface textures is closely related to texture dimensions and similar textures receive uniform ratings $[53,54]$.

\subsection{CONCLUSIONS}

Tactile friction is greatly influenced by the real contact area due to the predominant adhesion. Deterministic surfaces allow a significant reduction in friction if the skin remains in the asperity contact state. Dense structures showed a reduction in friction coefficient by a factor of at least 3. As the skin deforms past the texture feature heights, the transition to the full contact leads to a noticeable increase in friction. 
Observation of the same effect allows estimation of skin deformation and its use for numerical modelling.

Adhesive friction is responsible for the directional sliding dependance in elastic asymmetric texture designs. The results showed the feasibility of using asymmetric texture designs for tactile friction, which are based on the change in the contact area and not the deformation of the skin. Utilising elastomer materials, features can be designed to deform under shear forces, thus increasing the contact area and friction forces. The resulting difference in directional sliding highly depends on the material elastic modulus, feature dimensions and a contact state.

Friction measurements performed with a group of 10 volunteers showed that tactile friction can be normalised and compared quantitatively between individuals if the requirements for the adhesive friction are met. Furthermore, despite the personal differences, the range of the skin deformations was similar between participants. It suggests that deterministic surface designs remain functional for a group of people and independent of their personal skin parameter variations. 


\section{SURFACE DESIGN}

\subsection{VARIETY OF TEXTURES FOR TACTILITY}

\subsubsection{Background}

Surface design is well known to affect both tactile and visual properties of a product, which are generally discussed as an attribute of the material and finishing operations [6,107]. During the conceptual design stage, designers are reported to be more interested in the sensorial aspects of materials, and their intangible characteristics such as associations, emotions, and cultural meanings, than in material-related technical properties and associated manufacturing processes [108]. Surface texturing can further modify and possibly enhance the perceptual qualities of a surface and provide additional solutions. A method for selection of texture design and its dimensions, is however not yet available. To help bridge this gap in product design, the foundations of such a method are laid by applying the results of the current work to a review of tactility studies with respect to the correlation of texture feature dimensions, mechanical effects, and perceived properties. 


\subsubsection{Overview of current texture designs for tactile surfaces}

Deterministic textures can be divided into three typical groups by their design structure: a matrix of dimples, parallel-ridged surfaces, and evenly spaced bump features, with the latter two being widely represented in literature. Both bump and dimple textures provide a uniform reduction of surface area in contact and friction mostly independent from sliding direction. If a bump texture is sliding against a skin, local areas of contact on the skin are constantly changing, producing variable pressure and shear forces. On the contrary, for the crater surfaces there are regions of the skin which remain in continuous contact with the surface and, therefore, forces are continuous in these regions. Parallel ridged surfaces are often used to produce tactile friction dependent on sliding direction [83] and increase friction by finger deformation [78,82]. Depending on the sliding directions one of the two previously mentioned scenarios takes place: either skin undergoes iterative waveform loading or remains in permanent contact with the surface, inducing a constant shear force on the skin regions in contact.

Furthermore, specific texture shapes can be distinguished. From the review the following shapes were identified:

1. triangular shaped features, including conical features, sandpaper grains[43,109] and triangular ridges [82];

2. rectangular shaped features such as pillars [54,110] and rectangular ridges [49,78];

3. rounded profiles including spherical bump features $[85,88]$ and wavy ridge surfaces [51].

The texture dimensions are described by the lateral spacing between features $\lambda$, feature height $h_{a s p}$ and width $w_{a s p}$. For spherical-shaped features, the width is equal to the double radius of the feature $w_{a s p}=$ 
$2 R_{\text {asp }}$. The variety of the texture types requires generalization of the dimensional parameters to allow mapping in a single graph.

For the hemi-cylindrical ridge and spherical bump textures the critical normal load $\left(F_{\text {crit }}\right)$ at the macro scale, at which the contact transition from asperity to full contact state happens, is directly proportional to the texture parameters. Based on the Hertzian contact model, the critical normal load is directly related to the ridge height and spacing $F_{\text {crit }} \propto$ $h_{a s p} / \lambda$ for the hemi-cylindrical parallel ridged texture. A similar relationship was derived for the bump patterns $F_{\text {crit }} \propto \sqrt{h_{a s p}^{3} R_{a s p}} / \lambda^{2}$. These dependencies showed that the feature height and spacing are expected to have the highest impact on the contact state at the microscale. Furthermore, the height of the spherical features can be equated to their radius $h_{\text {asp }}=R_{\text {asp }}$ to reduce the number of parameters on the graph. It implies an aspect ratio for the features equal to $w_{a s p} / h_{a s p}=0.5$. On this basis, lateral spacing and feature radius were selected for the graph as geometric parameters.

To plot the textures studied in literature, the reported dimensions were converted to these two parameters where possible. For all parallel-ridged textures, the height was used as a secondary parameter. Bump features are represented in literature by pillars and spherical bumps. Considering the significance of the height for the bump features, their effective feature radius was estimated as $R_{\text {asp }}=\sqrt{h_{\text {asp }}^{3} w_{\text {asp }}}$ for the purpose of data-plotting. For the dimple surfaces, the radius or half-width of the crater was used.

The contact area for the textures with a spacing below $200 \mu \mathrm{m}$ can be calculated with the proposed contact model to represent the boundary condition between asperity and full contact states. The calculation results significantly varied for different texture types; the presented plot was chosen to be based on the spherical bump texture matrix, due to the predominance of this pattern in literature. 
Based on the reported friction values and observations in the corresponding papers, one of the three contact states was assumed and assigned to each texture, i.e.: asperity contact, transitive state, or full contact. For the perception studies the same attributes were applied that are: smooth/rough, textured, and slippery/sticky surfaces, respectively. Significant changes in tactile friction and perception can therefore be observed independent of the responsible mechanism. Normal loads applied to the finger pad in the observed literature vary from 0.1 to $25 \mathrm{~N}$. However, most of the studies are performed at a lower normal load range, i.e., between 0.5 and $2 \mathrm{~N}$; therefore, the data for the plot was collected at $1 \mathrm{~N}$ load, if available.

\subsection{PERCEPTION AND FRICTION EFFECTS}

A generalised texture design map for up to a millimetre spacing range is constructed and shown in Figure 5.1. The resulted map serves multiple purposes:

(1) It presents the main feature dimensions of the textures presented in Chapter 4 and in the available literature. Likewise, it highlights the areas yet to be analysed through the limited coverage of datapoints.

(2) It predicts the relation between the specific attributes of tactility with friction effects to the feature dimensions, based on reported tactility and friction measurements in literature. The specific attributes are: smooth/rough, sticky/slippery and textured.

(3) It demarks the boundary transition region for the spherical bump texture type, by the $200 \mu \mathrm{m}$ line for feature spacing. 


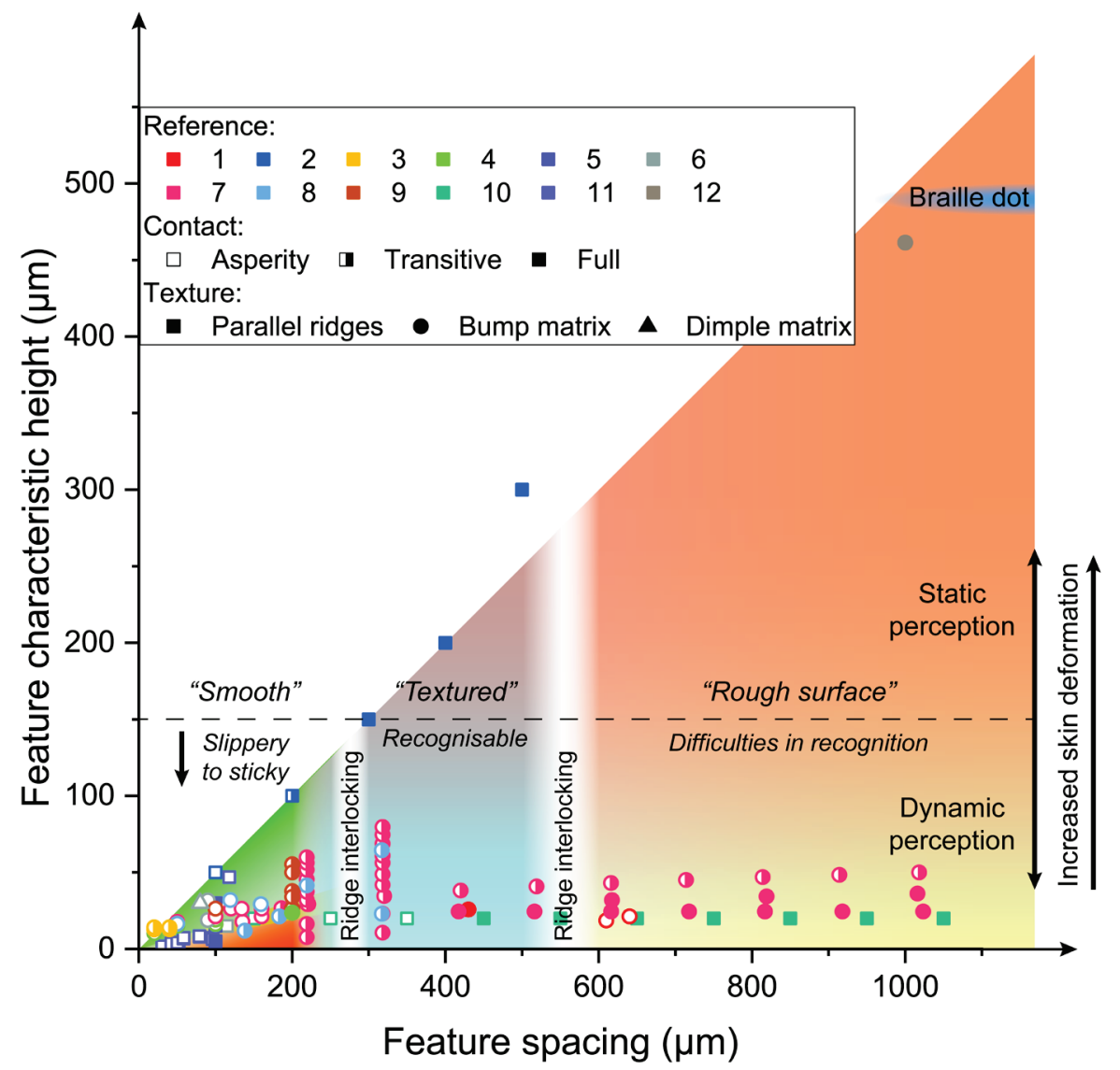

FIGURE 5.1 Friction and perception mapping to texture feature dimensions. References from the legend are given in Table 5.1.

TABLE 5.1 Legend graph to literature reference.

\begin{tabular}{|cccccccccccc|}
\hline 1 & 2 & 3 & 4 & 5 & 6 & 7 & 8 & 9 & 10 & 11 & 12 \\
\hline$[86]$ & {$[82]$} & {$[88]$} & {$[87]$} & {$[83]$} & {$[84]$} & {$[54]$} & {$[53]$} & {$[111]$} & {$[49]$} & {$[51]$} & {$[110]$} \\
\hline
\end{tabular}


With an increase of the asperity radius, the bulk deformation of the finger pad increases $[78,81,110,112]$. With a typical height of $0.5 \mathrm{~mm}$, a feature used in the Braille alphabet is located on the larger scale of the graph. Sliding against Braille features clearly shows the increase in tactile friction due to deformation [85]. Feature sizes below $150 \mu \mathrm{m}$ need a sliding movement to distinguish tactile sensations, as indicated by the dashedline in Figure 5.1, see also the work of Hollins and Risner [37].

Along the spacing scale, the graph is roughly divided into three regions by the feature spacing. The typical spacing between fingerprint ridges corresponds to the range around 500 to $600 \mu \mathrm{m}[11,87]$. If a textured surface wavelength closely matches that of the fingerprint, multiple asperities fit between the skin furrows along the sliding path. While the effect has not been studied yet, it is expected to cause stick-slip behaviour and increase of friction, further promoted by asperity size and asperity slope. A similar interlocking effect, but to a lesser extent, is predicted between 250 and $300 \mu \mathrm{m}$ as a harmonic wavelength of the fingerprint ridges. Furthermore, Fagiani et al. $[45,48]$ studied the relation between fingerprint wavelength, vibration spectra and perception. They concluded that when the topographic wavelength is much larger than that of the fingerprint, the vibration is masked, and the surface is perceived through a pressure distribution as in static exploratory procedure for tactile perception. Textures having these feature distributions are perceived as rough [53,54]. It is also reported that volunteers have difficulties differentiating between these feature distributions [49].

In the spacing range between 300 and $500 \mu \mathrm{m}$, the fingerprint ridge is supported mainly by a single asperity, which is not a stable condition and could explain the mixed perception reports in this range. On the higher part of the spacing range $(>400 \mu \mathrm{m})$ the surfaces were considered rough, but with reduction of spacing the surfaces are described as vibrating [54] and the successful recognition rate also increases [49]. 
At spacings below approximately $200 \mu \mathrm{m}$, each ridge is supported by two or more asperities, which significantly reduces the probability of the interlocking mechanism. In this region the friction is based mainly on adhesion, which is directly correlated with the contact area. A previously calculated contact area in Figure 3.4 is integrated into this region in green to highlight the differences in the estimated transition (yellow) and full contact (orange) states, as shown in Figure 5.2. Surfaces which fall in this green region are often described by volunteers as silky, smooth or slippery $[51,53,54]$. With reduction of the feature radius the tactile friction reduces until the deformation reaches the asperity height. Subsequently, friction increases and affects the surface perception.

The green area that is indicated in the Figure 5.2 guides product and engineering designers in designing surface textures within the scope of this thesis, aiming at controlling and enhancing tactile perception through modification of the frictional behaviour. Within this area the texture design fulfils the functionality that is designed, outside the area unwanted effects are expected to occur that reduces the effectiveness of the texture and consequently reduces the designed functionality. In fact, the proposed contact model for microscale contact is confirmed by the diagram as well, as it is in line with the data reported in literature and supports the tactile and frictional behaviour observed by other researchers $[53,54,84,87,88]$. 


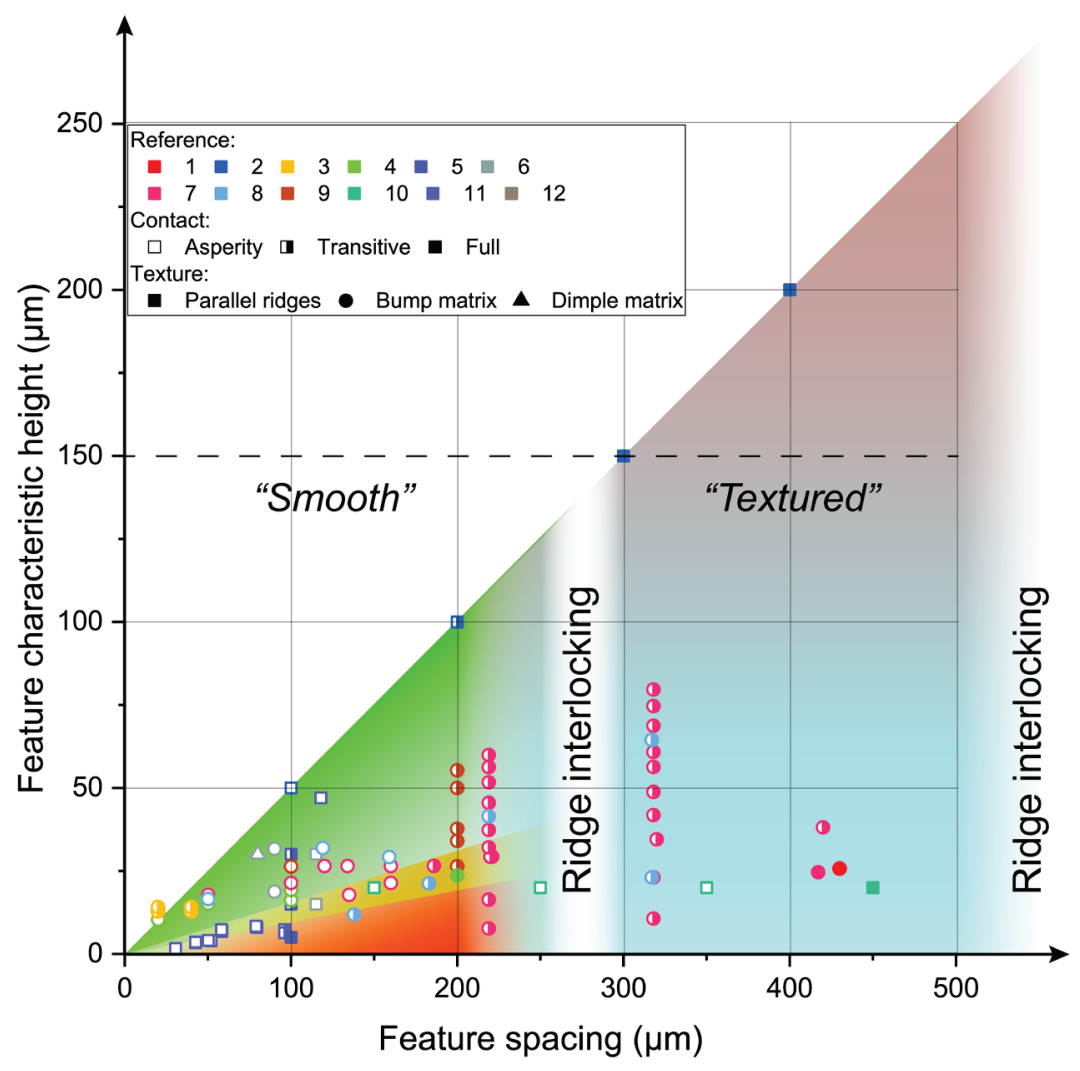

FIGURE 5.2 Friction and perception mapping to texture feature dimensions. Spacing up to $500 \mu \mathrm{m}$. References from the legend are given in Table 5.1. 
Clearly, the diagram can also be used to design other tactile sensations by surface textures, although the use is limited to the selected tactile attributes smooth/rough, sticky/slippery and textured. Within the presented texture classes, the methods to formulate a feature radius and feature spacing is given in detail. New texture designs, however, require an accompanying approach in formulating a characteristic texture length perpendicular to the direction of sliding and a characteristic texture length parallel to the direction of sliding.

\subsection{CONCLUSIONS}

A generalised texture design map for up to a millimetre spacing range is developed and presented. The texture designs presented in this thesis and reported in literature provide only a limited texture coverage as the field is developing.

The texture design map can be used to design surface textures within the scope of the current work, aiming at controlling and enhancing tactile perception through modification of the frictional behaviour.

The proposed contact model for microscale contact is in line with the data reported in literature and supports the tactile and frictional behaviour observed by other researchers. 


\section{CONCLUSIONS AND OUTLOOK}

\subsection{CONCLUSIONS}

Tactile perception can be controlled through modification of the frictional behaviour in finger pad - surface interactions by changing the topography of the surface. As part of a systematic approach, an asperity and texture design was selected for this research. A contact model was developed to predict finger pad contact area on micro- and macro- scales. The model was applied to understand the role of component parts in a tactile tribological system - namely to estimate skin elastic modulus, characterise skin deformation and determine the role of varying surface texture dimensions. The influence of texture parameters and of individual finger properties was evaluated through experimental and numerical studies. Finally, texture dimensions were related to friction observed in this thesis and perception and friction effects taken from literature, which resulted in the foundations of a design map.

Numerical methods

- A contact model, which combines analytical and numerical approaches, was developed to predict, and calculate the contact area for the finger pad in contact with micro-textured surfaces. Implementation of the boundary element method allowed to quantify the contact transition effect on the contact area based on 
the change from an asperity supported state to full contact with the surface.

- The proposed model was applied to estimate the effective elastic modulus of the skin appropriate for the micro-scale system based on the observation of the contact transition through tactile friction measurements. The values were found in the range between 0.2 and $0.5 \mathrm{MPa}$, which is in line with the reported values obtained from the fingerprint ridge deformation.

- Simulation results for a simplified spherical bump texture case showed that the contact state can be defined by a non-dimensional texture parameter based on the feature height $h_{a s p}$, radius $R_{a s p}$ and spacing $\lambda: k_{t}=\sqrt{h_{a s p}^{3} R_{a s p}} / \lambda^{2}$. For a finger pad contact at a normal load of $1 \mathrm{~N}$ against materials with significantly higher stiffness than that of the skin, the texture parameter value of $k_{t}<0.03$ was estimated as a condition for the contact transition to occur.

\subsubsection{Friction measurements}

- When a finger pad is sliding against a textured surface, the contact state significantly influenced the observed tactile friction, confirming that the friction has a predominantly adhesive nature. Deterministic surfaces, which remained in asperity contact showed a considerable reduction of the friction coefficient. The difference in friction values allowed to determine the contact state and use the contact transition state as an indication of skin deformation on the asperity scale.

- Directional dependence of tactile friction was achieved with ellipsoidal asperity textures produced on elastic materials. The results showed the feasibility of the asymmetric texture designs 
for tactile friction, which was based on the change in the contact area and not the deformation of the skin.

- Friction measurements performed with a group of volunteers showed that a reference sample can be used to normalise and compare values between individuals. Furthermore, despite the variation between individual finger parameters, the participants showed a comparable range for the skin micro-deformation, suggesting that the same textured surfaces have a similar functional performance for a group of people.

\subsubsection{Texture design}

- A texture design map to make a connection between texture dimensions and effects attributed to tactile interaction has been presented. The diagram also serves to highlight areas for development in the field of tactile surface design as the textures reported in literature cover the dimensional space only partially.

- The diagram can be used as a reference for surface texture design within the scope of the work, aiming to control and predict tactile frictional behaviour.

- The predictions obtained with the proposed model for the contact between finger pad and microscale textures are in accordance with reports from literature. 


\subsection{OUTLOOK}

Understanding of the mechanisms behind finger pad friction and perception is not only valuable for the improvement of consumer products, but serves as a stepping stone towards replication of tactile senses for other areas of application. Theoretically, a physical surface can be simulated by a haptic device with a dynamic array of locally induced normal and tangential forces. Such technology could serve as a significant improvement of the existing virtual reality technology. Furthermore, the development of electronic sensors, capable of interaction at similar resolution as a human skin will lead to advancements in prosthetic implants, remote surgery, and robotic automation.

In a simplified form, a conditional change of the surface parameters can be used to manipulate tactile perception and friction. A binary control operation can be achieved by changing a surface topography through active or passive means, e.g., it could be a material reaction to an external electronic signal, change in humidity or applied pressure. The perceived effect of such variation can be increased by designing a texture with dimensions close to the boundary condition, e.g., the contact state described in this work.

The described asperity contact states can be used to design 'active' surfaces. A plausible example of such a surface could be a microtextured surface, which can be locally actuated in such a way that, the texture topography can be controlled statically or dynamically. Topographic actuation in this way can be sufficient to change the tactile behaviour from full to asperity contact and vice versa. 


\section{BIBILOGRAPHY}

[1] C.L. Mackenzie, T. Iberall, Chapter 3. Serial Order in Prehension, in: Elsevier Science B.V., Amsterdam, 1994: pp. 49-61.

[2] R.S. Johansson, G. Westling, Signals in tactile afferents from the fingers eliciting adaptive motor responses during precision grip, Exp. Brain Res. 66 (1987) 141-154.

[3] C.J. Winstein, J.H. Abbs, D. Petashnick, Influences of object weight and instruction on grip force adjustments, Exp. Brain Res. 87 (1991) 465-469.

[4] W.M. Bergmann Tiest, Tactual perception of material properties, Vision Res. 50 (2010) 2775-2782.

[5] M. Holliins, R. Faldowski, S. Rao, F. Young, Perceptual dimensions of tactile surface texture: A multidimensional scaling analysis, Percept. Psychophys. 54 (1993) 697-705.

[6] H. Zuo, T. Hope, M. Jones, Tactile Aesthetics of Materials and Design, Elsevier, 2014.

[7] L. Veelaert, E. Du Bois, I. Moons, E. Karana, Experiential characterization of materials in product design: A literature review, Mater. Des. 190 (2020) 108543.

[8] K.E. Overvliet, S. Soto-Faraco, I can't believe this isn't wood! An investigation in the perception of naturalness, Acta Psychol. (Amst). 136 (2011) 95-111.

[9] K. Chopra, D. Calva, M. Sosin, K.K. Tadisina, A. Banda, C. De La Cruz, M.R. Chaudhry, T. Legesse, C.B. Drachenberg, P.N. Manson, M.R. Christy, A comprehensive examination of topographic thickness of skin in the human face, Aesthetic Surg. J. 35 (2015) 1007-1013.

[10] H. Fruhstorfer, U. Abel, C.D. Garthe, A. Knüttel, Thickness of the stratum corneum of the volar fingertips, Clin. Anat. 13 (2000) 429-433.

[11] X. Liu, Z. Lu, R. Lewis, M.J. Carré, S.J. Matcher, Feasibility of using optical coherence tomography to study the influence of skin structure on finger friction, Tribol. Int. 63 (2013) 34-44.

[12] O.S. Dinc, C.M. Ettles, S.J. Calabrese, H. a. Scarton, O.S. Dinç, C.M. Ettles, S.J. Calabrese, H. a. Scarton, Some parameters affecting tactile friction, J. 
Tribol. 113 (1991) 512-517.

[13] M.J. Adams, S.A. Johnson, P. Lefèvre, V. Lévesque, V. Hayward, T. André, J.L.L. Thonnard, Finger pad friction and its role in grip and touch, J. R. Soc. Interface. 10 (2013) 20120467.

[14] S. Bochereau, B. Dzidek, M. Adams, V. Hayward, Characterizing and imaging gross and real finger contacts under dynamic loading, IEEE Trans. Haptics. 10 (2017) 456-466.

[15] B. Dzidek, S. Bochereau, S. Johnson, V. Hayward, M. Adams, Frictional dynamics of finger pads are governed by four length-scales and two timescales, in: 2016 IEEE Haptics Symp., IEEE, 2016: pp. 161-166.

[16] A. Abdouni, M. Djaghloul, C. Thieulin, R. Vargiolu, C. Pailler-Mattei, H. Zahouani, Biophysical properties of the human finger for touch comprehension: Influences of ageing and gender, R. Soc. Open Sci. 4 (2017).

[17] T. Gambichler, R. Matip, G. Moussa, P. Altmeyer, K. Hoffmann, In vivo data of epidermal thickness evaluated by optical coherence tomography: Effects of age, gender, skin type, and anatomic site, J. Dermatol. Sci. 44 (2006) 145-152.

[18] P. Cornuault, L. Carpentier, M. Bueno, J. Cote, G. Monteil, Influence of physico-chemical, mechanical and morphological fingerpad properties on the frictional distinction of sticky/slippery surfaces, J. R. Soc. Interface. 12 (2015) 20150495.

[19] N.K. Veijgen, M.A. Masen, E. Van Der Heide, Relating friction on the human skin to the hydration and temperature of the skin, Tribol. Lett. 49 (2013) 251-262.

[20] J. van Kuilenburg, M.A. Masen, E. van der Heide, Contact modelling of human skin: What value to use for the modulus of elasticity?, Proc. Inst. Mech. Eng. Part J J. Eng. Tribol. 227 (2012) 349-361.

[21] C. Edwards, R. Marks, Evaluation of biomechanical properties of human skin, Clin. Dermatol. 13 (1995) 375-380.

[22] C. Pailler-Mattei, S. Pavan, R. Vargiolu, F. Pirot, F. Falson, H. Zahouani, Contribution of stratum corneum in determining bio-tribological properties of the human skin, Wear. 263 (2007) 1038-1043.

[23] Y. Yuan, R. Verma, Measuring microelastic properties of stratum corneum, Colloids Surfaces B Biointerfaces. 48 (2006) 6-12.

[24] M. Geerligs, L. van Breemen, G. Peters, P. Ackermans, F. Baaijens, C. Oomens, In vitro indentation to determine the mechanical properties of epidermis, J. Biomech. 44 (2011) 1176-1181.

[25] H. Zahouani, C. Pailler-Mattei, B. Sohm, R. Vargiolu, V. Cenizo, R. Debret, Characterization of the mechanical properties of a dermal equivalent compared with human skin in vivo by indentation and static friction tests, Ski. Res. Technol. 15 (2009) 68-76.

[26] S.A. Johnson, D.M. Gorman, M.J. Adams, B.J. Briscoe, The friction and lubrication of human stratum corneum., Tribol. Ser. 25 (1993) 663-672. 
[27] C.-Y. Chen, C.-A. Yu, T.-F. Hong, Y.-L. Chung, W.-L. Li, Contact and frictional properties of stratum corneum of human skin, Biosurface and Biotribology. 1 (2015) 62-70.

[28] C. Pailler-Mattei, S. Bec, H. Zahouani, In vivo measurements of the elastic mechanical properties of human skin by indentation tests, Med. Eng. Phys. 30 (2008) 599-606.

[29] A. Delalleau, G. Josse, J.M. Lagarde, H. Zahouani, J.M. Bergheau, Characterization of the mechanical properties of skin by inverse analysis combined with the indentation test, J. Biomech. 39 (2006) 1603-1610.

[30] Q. Wang, V. Hayward, In vivo biomechanics of the fingerpad skin under local tangential traction, J. Biomech. 40 (2007) 851-860.

[31] R.S. Johansson, A.B. Vallbo, Tactile sensibility in the human hand: relative and absolute densities of four types of mechanoreceptive units in glabrous skin., J. Physiol. 286 (1979) 283-300.

[32] R.S. Johansson, J.R. Flanagan, Coding and use of tactile signals from the fingertips in object manipulation tasks, Nat. Rev. Neurosci. 10 (2009) 345359.

[33] R.J. Seitz, P.E. Roland, C. Bohm, T. Greitz, S. Stone-Elander, Somatosensory Discrimination of Shape: Tactile Exploration and Cerebral Activation, Eur. J. Neurosci. 3 (1991) 481-492.

[34] A.M. Smith, G. Gosselin, B. Houde, Deployment of fingertip forces in tactile exploration, Exp. Brain Res. 147 (2002) 209-218.

[35] M.A. Plaisier, W.M. Bergmann Tiest, A.M.L. Kappers, Haptic pop-out in a hand sweep, Acta Psychol. (Amst). 128 (2008) 368-377.

[36] L.E.M. Grierson, H. Carnahan, Manual exploration and the perception of slipperiness, Percept. Psychophys. 68 (2006) 1070-1081.

[37] M. Hollins, S.R. Risner, Evidence for the duplex theory of tactile texture perception, Percept. Psychophys. 62 (2000) 695-705.

[38] W.M. Bergmann Tiest, A.M.L. Kappers, Analysis of haptic perception of materials by multidimensional scaling and physical measurements of roughness and compressibility, Acta Psychol. (Amst). 121 (2006) 1-20.

[39] L. Skedung, K. Danerlöv, U. Olofsson, M. Aikala, K. Niemi, J. Kettle, M.W. Rutland, Finger friction measurements on coated and uncoated printing papers, Tribol. Lett. 37 (2010) 389-399.

[40] G. Robles-De-La-Torre, V. Hayward, Force can overcome object geometry in the perception of shape through active touch, Nature. 412 (2001) 445448.

[41] A. Barrea, B.P. Delhaye, P. Lefèvre, J.L. Thonnard, Perception of partial slips under tangential loading of the fingertip, Sci. Rep. 8 (2018) 7032.

[42] T. Yoshioka, S.J. Bensmaïa, J.C. Craig, S.S. Hsiao, Texture perception through direct and indirect touch: An analysis of perceptual space for tactile textures in two modes of exploration, Somatosens. Mot. Res. 24 (2007) 53-70. 
[43] X. Zhou, J.L. Mo, Y.Y. Li, J.Y. Xu, X. Zhang, S. Cai, Z.M. Jin, Correlation between tactile perception and tribological and dynamical properties for human finger under different sliding speeds, Tribol. Int. 123 (2018) 286295.

[44] R. Fagiani, F. Massi, E. Chatelet, Y. Berthier, A. Sestieri, Experimental analysis of friction-induced vibrations at the finger contact surface, Proc. Inst. Mech. Eng. Part J J. Eng. Tribol. 224 (2010) 1027-1035.

[45] R. Fagiani, F. Massi, E. Chatelet, Y. Berthier, A. Akay, Tactile perception by friction induced vibrations, Tribol. Int. 44 (2011) 1100-1110.

[46] R. Fagiani, F. Massi, E. Chatelet, J.P. Costes, Y. Berthier, Contact of a finger on rigid surfaces and textiles: Friction coefficient and induced vibrations, Tribol. Lett. 48 (2012) 145-158.

[47] R. Fagiani, M. Barbieri, Modelling of finger-surface contact dynamics, Tribol. Int. 74 (2014) 130-137.

[48] R. Fagiani, M. Barbieri, A contact mechanics interpretation of the duplex theory of tactile texture perception, Tribol. Int. 101 (2016) 49-58.

[49] I. Cesini, J.D. Ndengue, E. Chatelet, J. Faucheu, F. Massi, Correlation between friction-induced vibrations and tactile perception during exploration tasks of isotropic and periodic textures, Tribol. Int. 120 (2018) 330-339.

[50] L. Skedung, M. Arvidsson, J.Y. Chung, C.M. Stafford, B. Berglund, M.W. Rutland, Feeling small: Exploring the tactile perception limits, Sci. Rep. 3 (2013).

[51] M. Arvidsson, L. Ringstad, L. Skedung, K. Duvefelt, M.W. Rutland, Feeling fine - the effect of topography and friction on perceived roughness and slipperiness, Biotribology. 11 (2017) 92-101.

[52] K. Duvefelt, U. Olofsson, C.M. Johannesson, L. Skedung, Model for contact between finger and sinusoidal plane to evaluate adhesion and deformation component of friction, Tribol. Int. 96 (2016) 389-394.

[53] V. Massimiani, B. Weiland, E. Chatelet, P.H. Cornuault, J. Faucheu, F. Massi, The role of mechanical stimuli on hedonistic and topographical discrimination of textures, Tribol. Int. 143 (2020) 106082.

[54] J. Faucheu, B. Weiland, M. Juganaru-Mathieu, A. Witt, P.H. Cornuault, Tactile aesthetics: Textures that we like or hate to touch, Acta Psychol. (Amst). 201 (2019) 102950.

[55] B.K. Chao, H.H. Cheng, L.W. Nien, M.J. Chen, T. Nagao, J.H. Li, C.H. Hsueh, Anti-reflection textured structures by wet etching and island lithography for surface-enhanced Raman spectroscopy, Appl. Surf. Sci. 357 (2015) 615621.

[56] S. Bruening, K. Du, M. Jarczynski, A. Gillner, High-throughput micromachining with ultrashort pulsed lasers and multiple spots, J. Laser Appl. 32 (2020) 012003.

[57] A. Arslan, H.H. Masjuki, M.A. Kalam, M. Varman, R.A. Mufti, M.H. Mosarof, L.S. Khuong, M.M. Quazi, Surface Texture Manufacturing Techniques and 
Tribological Effect of Surface Texturing on Cutting Tool Performance: A Review, Crit. Rev. Solid State Mater. Sci. 41 (2016) 447-481.

[58] M. Mezera, M. van Drongelen, G.R.B.E. Römer, Laser-Induced Periodic Surface Structures (LIPSS) on polymers processed with picosecond laser pulses, J. Laser Micro Nanoeng. 13 (2018) 105-116.

[59] T. Velten, F. Bauerfeld, H. Schuck, S. Scherbaum, C. Landesberger, K. Bock, Roll-to-roll hot embossing of microstructures, Microsyst. Technol. 17 (2011) 619-627.

[60] L. Peng, Y. Deng, P. Yi, X. Lai, Micro hot embossing of thermoplastic polymers: a review, J. Micromechanics Microengineering. 24 (2014) 13001.

[61] M. Calaon, D. Quagliotti, G. Tosello, Surface Replication in Micro Injection Molding, First Edit, Carl Hanser Verlag GmbH \& Co. KG, 2018.

[62] C. Liedert, L. Rannaste, A. Kokkonen, O.H. Huttunen, R. Liedert, J. Hiltunen, L. Hakalahti, Roll-to-Roll Manufacturing of Integrated Immunodetection Sensors, ACS Sensors. 5 (2020) 2010-2017.

[63] A.W.J. de Gee, G.W. Rowe, Glossary of Terms and Definitions in the Field of Friction, Wear, and Lubrication: Tribology, Organization for Economic Co-operation and Development, Paris, 1969.

[64] H. Czichos, ed., 2 The System Concept, in: Tribol. Ser., 1978: pp. 14-23.

[65] G. Salomon, Application of Systems Thinking to Tribology, A S L E Trans. 17 (1974) 295-299.

[66] M. Klaassen, D.J. Schipper, M.A. Masen, Influence of the relative humidity and the temperature on the in-vivo friction behaviour of human skin, Biotribology. 6 (2016) 21-28.

[67] C.P. Hendriks, S.E. Franklin, Influence of surface roughness, material and climate conditions on the friction of human skin, Tribol. Lett. 37 (2010) 361-373.

[68] S. Derler, M. Preiswerk, G.M. Rotaru, J.P. Kaiser, R.M. Rossi, Friction mechanisms and abrasion of the human finger pad in contact with rough surfaces, Tribol. Int. 89 (2015) 119-127.

[69] M.J. Adams, B.J. Briscoe, S.A. Johnson, Friction and lubrication of human skin, Tribol. Lett. 26 (2007) 239-253.

[70] S. Derler, L.C. Gerhardt, A. Lenz, E. Bertaux, M. Hadad, Friction of human skin against smooth and rough glass as a function of the contact pressure, Tribol. Int. 42 (2009) 1565-1574.

[71] S. Derler, G.M. Rotaru, Stick-slip phenomena in the friction of human skin, Wear. 301 (2013) 324-329.

[72] J.A. Greenwood, H. Minshall, D. Tabor, Hysteresis Losses in Rolling and Sliding Friction, Proc. R. Soc. A Math. Phys. Eng. Sci. 259 (1961) 480-507.

[73] C. Pailler-Mattei, S. Nicoli, F. Pirot, R. Vargiolu, H. Zahouani, A new approach to describe the skin surface physical properties in vivo, Colloids Surfaces B Biointerfaces. 68 (2009) 200-206. 
[74] A.E. Kovalev, K. Dening, B.N.J. Persson, S.N. Gorb, Surface topography and contact mechanics of dry and wet human skin, Beilstein J. Nanotechnol. 5 (2014) 1341-1348.

[75] B.N.J. Persson, A. Kovalev, S.N. Gorb, Contact Mechanics and Friction on Dry and Wet Human Skin, Tribol. Lett. 50 (2013) 17-30.

[76] B.N.J. Persson, Capillary adhesion between elastic solids with randomly rough surfaces, J. Phys. Condens. Matter. 20 (2008).

[77] S.E. Tomlinson, R. Lewis, X. Liu, C. Texier, M.J. Carré, Understanding the friction mechanisms between the human finger and flat contacting surfaces in moist conditions, Tribol. Lett. 41 (2011) 283-294.

[78] S.E. Tomlinson, R. Lewis, M.J. Carré, The effect of normal force and roughness on friction in human finger contact, Wear. 267 (2009) 13111318.

[79] L. Skedung, K. Danerlöv, U. Olofsson, C. Michael Johannesson, M. Aikala, J. Kettle, M. Arvidsson, B. Berglund, M.W. Rutland, Tactile perception: Finger friction, surface roughness and perceived coarseness, Tribol. Int. 44 (2011) 505-512.

[80] G.P. Chimata, C.J. Schwartz, Investigation of friction mechanisms in finger pad sliding against surfaces of varying roughness, Biotribology. 3 (2015) $11-19$.

[81] S.E. Tomlinson, R. Lewis, M.J. Carré, S.E. Franklin, Human finger friction in contacts with ridged surfaces, Wear. 301 (2013) 330-337.

[82] S.E. Tomlinson, M.J. Carré, R. Lewis, S.E. Franklin, Human finger contact with small, triangular ridged surfaces, Wear. 271 (2011) 2346-2353.

[83] S. Zhang, A. Rodriguez Urribarri, M. Morales Hurtado, X. Zeng, E. van der Heide, The role of the sliding direction against a grooved channel texture on tool steel: An experimental study on tactile friction, Int. J. Solids Struct. 56 (2015) 53-61.

[84] S. Zhang, X. Zeng, D.T.A. Matthews, A. Igartua, E. Rodriguez-Vidal, J.C. Fortes, E. van der Heide, Texture design for light touch perception, Biosurface and Biotribology. 3 (2017) 25-34.

[85] M.A. Darden, C.J. Schwartz, Skin tribology phenomena associated with reading braille print: The influence of cell patterns and skin behavior on coefficient of friction, Wear. 332-333 (2015) 734-741.

[86] T.H.C. Childs, B. Henson, Human tactile perception of screen-printed surfaces: Self-report and contact mechanics experiments, Proc. Inst. Mech. Eng. Part J J. Eng. Tribol. 221 (2007) 427-441.

[87] J. Van Kuilenburg, M.A. Masen, E. van der Heide, The role of the skin microrelief in the contact behaviour of human skin: Contact between the human finger and regular surface textures, Tribol. Int. 65 (2013) 81-90.

[88] J. Van Kuilenburg, M.A. Masen, M.N.W. Groenendijk, V. Bana, E. van der Heide, An experimental study on the relation between surface texture and tactile friction, Tribol. Int. 48 (2012) 15-21. 
[89] E.R. Serina, C.D. Mote, D. Rempel, Force response of the fingertip pulp to repeated compression - Effects of loading rate, loading angle and anthropometry, J. Biomech. 30 (1997) 1035-1040.

[90] X. Liu, M.J. Carré, Q. Zhang, Z. Lu, S.J. Matcher, R. Lewis, Measuring contact area in a sliding human finger-pad contact, Ski. Res. Technol. 24 (2018) 31-44.

[91] M. Wiertlewski, R.F. Friesen, J.E. Colgate, Partial squeeze film levitation modulates fingertip friction, Proc. Natl. Acad. Sci. U. S. A. 113 (2016) 92109215.

[92] K.B. Duvefelt, U.L.O. Olofsson, C.M.J. Johannesson, Towards simultaneous measurements of skin friction and contact area: Results and experiences, Proc. Inst. Mech. Eng. Part J J. Eng. Tribol. 229 (2015) 230-242.

[93] B.M. Dzidek, M.J. Adams, J.W. Andrews, Z. Zhang, S.A. Johnson, Contact mechanics of the human finger pad under compressive loads., J. R. Soc. Interface. 14 (2017) 20160935.

[94] T. Soneda, K. Nakano, Investigation of vibrotactile sensation of human fingerpads by observation of contact zones, Tribol. Int. 43 (2010) 210-217.

[95] A.C. Rodríguez Urribarrí, E. van der Heide, X. Zeng, M.B. de Rooij, Modelling the static contact between a fingertip and a rigid wavy surface, Tribol. Int. 102 (2016) 114-124.

[96] H.T. Lin, T.F. Hong, W.L. Li, Grip performance affected by water-induced wrinkling of fingers, Tribol. Lett. 58 (2015) 1-9.

[97] F. Shao, T.H.C. Childs, C.J. Barnes, B. Henson, Finite element simulations of static and sliding contact between a human fingertip and textured surfaces, Tribol. Int. 43 (2010) 2308-2316.

[98] K.L. Johnson, Contact Mechanics, Cambridge University Press, Cambridge, 1985.

[99] G.K. Nikas, Boussinesq-Cerruti functions and a simple technique for substantial acceleration of subsurface stress computations in elastic halfspaces, Proc. Inst. Mech. Eng. Part J J. Eng. Tribol. 220 (2006) 19-28.

[100] W.W. Chen, Q.J. Wang, A Numerical Static Friction Model for Spherical Contacts of Rough Surfaces, Influence of Load, Material, and Roughness, J. Tribol. 131 (2009) 021402.

[101] J. Cesbron, H.P. Yin, F. Anfosso-Lédée, D. Duhamel, D. Le Houédec, Z.Q. Feng, Numerical and experimental study of multi-contact on an elastic half-space, Int. J. Mech. Sci. 51 (2009) 33-40.

[102] M.F. Leyva-Mendivil, J. Lengiewicz, G. Limbert, Skin friction under pressure. The role of micromechanics, Surf. Topogr. Metrol. Prop. 6 (2018) 014001.

[103] J. Ilg, S.J. Rupitsch, A. Sutor, R. Lerch, Determination of dynamic material properties of silicone rubber using one-point measurements and finite element simulations, IEEE Trans. Instrum. Meas. 61 (2012) 3031-3038.

[104] S. Brüning, G. Jenke, K. Du, A. Gillner, High precision laser processing of steel surfaces with sub-ns-lasers, Phys. Procedia. 56 (2014) 919-926. 
[105] M.A. Darden, C.J. Schwartz, Investigation of friction mechanisms during the sliding of elastomers against hard parallel-ridge textures, Tribol. Int. 63 (2013) 2-7.

[106] N.K. Veijgen, M.A. Masen, E. van der Heide, Variables influencing the frictional behaviour of in vivo human skin, J. Mech. Behav. Biomed. Mater. 28 (2013) 448-461.

[107] H. Zuo, The Selection of Materials to Match Human Sensory Adaptation and Aesthetic Expectation in Industrial Design, METU J. Fac. Archit. 27 (2010) 301-319.

[108] E. Karana, P. Hekkert, P. Kandachar, Material considerations in product design: A survey on crucial material aspects used by product designers, Mater. Des. 29 (2008) 1081-1089.

[109] G.P. Chimata, C.J. Schwartz, Investigation of the Role of Diminishing Surface Area on Friction-Based Tactile Discrimination of Textures, Biotribology. 15 (2018) 1-8.

[110] G.D. Lamb, Tactile discrimination of textured surfaces: psychophysical performance measurements in humans, J. Physiol. 338 (1983) 551-565.

[111] D.A. Sergachev, D.T.A. Matthews, E. van der Heide, An Empirical Approach for the Determination of Skin Elasticity: Finger pad Friction against Textured Surfaces, Biotribology. 18 (2019) 100097.

[112] L.M. Vilhena, A. Ramalho, Friction behaviour of human skin rubbing against different textured polymeric materials obtained by a 3D printing micro-fabrication technique, Tribol. Trans. 0 (2019) 1-31. 


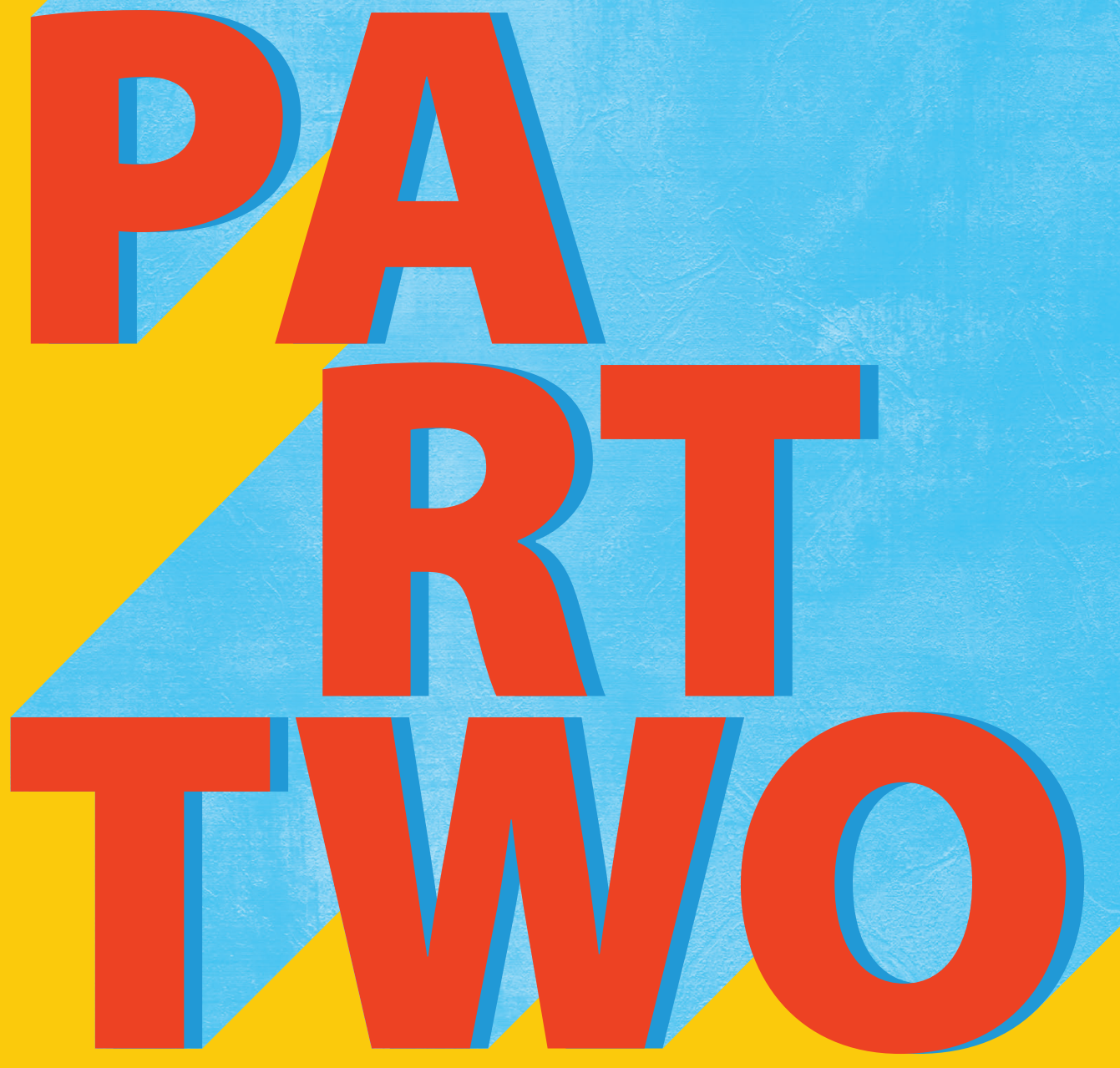





\section{PAPER A}

\section{An empirical approach for the determination of skin elasticity: finger pad friction against textured surfaces}

PUBLISHED AS:

BIOTRIBOLOGY, VOLUME 18, 2019, ARTICLE 100097

D. A. Sergachev, D. T. A. Matthews, E. van der Heide 



\section{NOMENCLATURE}

\begin{tabular}{|c|c|c|}
\hline symbol & description & units \\
\hline$A_{a}$ & $\begin{array}{l}\text { apparent contact area of a finger pad, measured } \\
\text { with a fingerprint scanner }\end{array}$ & $\mathrm{m}^{2}$ \\
\hline$a_{a c}$ & $\begin{array}{l}\text { equivalent radius of an apparent contact area, } \\
\text { calculated for an elastic material }\end{array}$ & $\mathrm{m}$ \\
\hline$A_{a c}$ & $\begin{array}{l}\text { apparent contact area of a finger pad, calculated } \\
\text { for an elastic material }\end{array}$ & $\mathrm{m}^{2}$ \\
\hline$a_{g}$ & $\begin{array}{l}\text { equivalent radius of a gross finger pad contact } \\
\text { area }\end{array}$ & $\mathrm{m}$ \\
\hline$A_{g}$ & $\begin{array}{l}\text { gross contact area of a finger pad, measured with } \\
\text { a fingerprint scanner }\end{array}$ & $\mathrm{m}^{2}$ \\
\hline$a_{g c}$ & $\begin{array}{l}\text { equivalent radius of a gross finger pad contact } \\
\text { area, calculated for an elastic material }\end{array}$ & $\mathrm{m}$ \\
\hline$A_{g c}$ & $\begin{array}{l}\text { gross contact area of a finger pad, calculated for } \\
\text { an elastic material }\end{array}$ & $\mathrm{m}^{2}$ \\
\hline$A_{r}$ & real contact area & $\mathrm{m}^{2}$ \\
\hline$a_{\text {trans }}$ & $\begin{array}{l}\text { equivalent radius of an apparent contact area in a } \\
\text { full contact state }\end{array}$ & $\mathrm{m}$ \\
\hline$A_{\text {trans }}$ & apparent contact area in a full contact state & $\mathrm{m}^{2}$ \\
\hline$E_{0}^{*}$ & effective Young's modulus in macro contact & $\mathrm{Pa}$ \\
\hline$E_{1}^{*}$ & effective Young's modulus in micro contact & $\mathrm{Pa}$ \\
\hline$E_{f p}$ & Young's modulus of a finger pad & $\mathrm{Pa}$ \\
\hline$E_{m}$ & Young's modulus of a material sample & $\mathrm{Pa}$ \\
\hline
\end{tabular}




\begin{tabular}{|c|c|c|}
\hline$E_{\text {smc }}$ & $\begin{array}{l}\text { effective skin Young's modulus in micro-scale } \\
\text { contact }\end{array}$ & $\mathrm{Pa}$ \\
\hline$F$ & applied normal load & $\mathrm{N}$ \\
\hline$F_{a d h}$ & interfacial adhesion friction force & $\mathrm{N}$ \\
\hline$F_{\text {crit }}$ & $\begin{array}{l}\text { normal load at which a transition to a full contact } \\
\text { state occurs }\end{array}$ & $\mathrm{N}$ \\
\hline$F_{d e f}$ & hysteresis friction force & $\mathrm{N}$ \\
\hline $\bar{F}_{i}$ & mean force acting on individual asperity & $\mathrm{N}$ \\
\hline$F_{\text {icrit }}$ & $\begin{array}{l}\text { critical force acting on individual asperity at } \\
\text { which a transition to a full contact state occurs }\end{array}$ & $\mathrm{N}$ \\
\hline$F_{i \max }$ & maximum force acting on individual asperity & $\mathrm{N}$ \\
\hline$F_{\text {tot }}$ & total friction force & $\mathrm{N}$ \\
\hline$h_{a s p}$ & height of an asperity & $\mathrm{m}$ \\
\hline$k$ & power-law coefficient for contact area & $\mathrm{m}^{2} \mathrm{~N}^{-m}$ \\
\hline$k_{a}$ & power-law coefficient for apparent contact area & $\mathrm{m}^{2} \mathrm{~N}^{-m_{a}}$ \\
\hline$k_{g}$ & power-law coefficient for gross contact area & $\mathrm{m}^{2} \mathrm{~N}^{-m_{g}}$ \\
\hline$m$ & power-law load index for contact area & - \\
\hline$m_{a}$ & power-law load index for apparent contact area & - \\
\hline$m_{g}$ & power-law load index for gross contact area & - \\
\hline$N$ & number of texture asperities in contact & - \\
\hline $\bar{p}$ & mean apparent contact pressure & $\mathrm{Pa}$ \\
\hline$p(r)$ & finger pad pressure radial distribution & $\mathrm{Pa}$ \\
\hline $\bar{p}_{a s p}$ & $\begin{array}{l}\text { mean apparent contact pressure in an asperity } \\
\text { contact state area }\end{array}$ & $\mathrm{Pa}$ \\
\hline
\end{tabular}


$\bar{p}_{\text {crit }} \quad$ critical apparent contact pressure at which a $\mathrm{Pa}$ transition to a full contact state occurs

$p_{\max } \quad$ maximum apparent contact pressure $\quad \mathrm{Pa}$

$\begin{array}{lll}\bar{p}_{r} & \text { mean real contact pressure } & \mathrm{Pa}\end{array}$

$\bar{p}_{\text {trans }} \quad$ mean apparent contact pressure in a full contact $\mathrm{Pa}$ state area

$r \quad$ radial distance from contact centre m

$R_{\text {asp }} \quad$ radius of an asperity tip m

$R_{e} \quad$ equivalent radius of a finger pad $\quad \mathrm{m}$

$\alpha \quad$ pressure coefficient

6 viscoelastic hysteresis loss fraction

$\delta \quad$ total micro-displacement of contacting surfaces $\mathrm{m}$

$\begin{array}{lll}\lambda & \text { spacing between texture asperities } \mathrm{m}\end{array}$

$\mu_{\text {adh }}$ interfacial adhesion coefficient of friction component

$\mu_{\text {def }} \quad$ hysteresis coefficient of friction component

$\mu_{\text {tot }} \quad$ total coefficient of friction

interfacial shear strength

$\tau_{0} \quad$ intrinsic interfacial shear strength $\quad \mathrm{Pa}$

$u_{f p} \quad$ Poisson's ratio of a finger pad

$u_{m} \quad$ Poisson's ratio of a sample material

$u_{s m c} \quad$ Poisson's ratio of skin in micro-scale contact 



\section{ABSTRACT}

Surface topography significantly influences tactile friction and perception. While friction forces can be reduced by surface texturing, selection of pattern dimensions is challenging due to the highly variable elastic modulus of the skin. This work proposes an empirical approach for the evaluation of the skin elasticity through surface transition from asperity to full contact state. To highlight the contact transition, two textures with evenly distributed identical micro asperities, but varying density, were moulded with several grades of silicone rubber. Dynamic friction coefficient measurements were performed during finger pad sliding against the textured samples with a range of normal loads up to 5 N. A combination of analytical and numerical contact models is used to explain the observed friction behaviour, estimate the development of contact area and calculate the effective elastic modulus of the skin at the micro-scale. Low density textures clearly indicate the transition to the full contact state, which is reflected in friction coefficient development, while high density textures remain in an asperity contact state, with significantly lower friction values. The effective Young's modulus is hereby estimated in the range of 0.2-0.5 MPa. Observed frictional behaviour is explained by the change in the apparent and real contact areas. The presented approach allows to study the influence of individual surface parameters on effective skin elastic modulus, which is essential for the development of functional surfaces with improved tactile perception.

Keywords

Skin tribology; tactile friction; Young's modulus; surface texture 


\section{INTRODUCTION}

Touch is one of the main senses that provides information for object perception. Tactile exploration, for example, allows object shape, edges and texture to be determined through mechanoreceptors located in the skin. Perceived surface feel is generally divided into three categories: hardness, roughness and stickiness [1], where the last two have been reported to be related to the topographical structure. Indeed, Skedung et al. [2] reported a positive relationship between perceived coarseness and surface roughness and stickiness feel was found to correlate with friction coefficients [3]. Moreover, surface discrimination was found to be influenced by surface texture to fingerprint wavelength ratio, which defines the induced vibrations during tactile exploration [4,5]. However, the overall understanding of touch and touch perception is not yet robust.

While haptic perception varies between individuals, it relies on the forces experienced during touch [6]. Friction forces arising from a finger pad sliding over a surface can be approximated by a two-term model, which describes the friction as a sum of the interfacial adhesion and deformation components $[7,8]$ :

$$
F_{t o t}=F_{a d h}+F_{\text {def }}
$$

In dry sliding against smooth surfaces, the adhesion term is predominant [9-11], however, with an increase of the surface roughness and asperity heights, the deformation component increases. Tomlinson et al. [12] observed a rise in the friction coefficient with increase of the machined roughness up to a certain point, after which it plateaued. For the triangular ridged surfaces with a height of $250 \mu \mathrm{m}$ the deformation term reached $10 \%$ of the total friction [13]. Moreover, a considerable influence of the interlocking friction component was reported, which became predominant at the ridge heights above $42.5 \mu \mathrm{m}$. Darden et al. [14] studied a sliding contact against spherical bumps with heights of 0.48 , 0.75 and $1.0 \mathrm{~mm}$. Interaction with a bump was separated from background signal as a differential friction coefficient for analysis. It was concluded that hysteretic deformation of the finger pad was the dominant mechanism in the observed rise of friction. 
As long as the adhesion term prevails, friction directly correlates to the real contact area. Therefore, a separation of the contacting bodies, caused by initial increase in the surface roughness, leads to a decrease in the friction coefficient for the dry skin contact $[9,15,16]$. Moreover, tactile friction can be controlled by deterministic roughness produced by surface structuring. Van Kuilenburg et al. $[17,18]$ tested microtextured surfaces with spherical tips of different radii and spacing, thus reducing real contact area and the observed friction values. It was concluded that a minimum value for the coefficient of friction can be achieved, before deformation and interlocking friction becomes predominant. Finding optimal texture dimensions to minimize skin friction is not a trivial task. The required surface geometry varies with the manufacturing process limitations, material properties of a product and its application conditions, given the system dependence of friction.

Mechanical properties of the skin vary due to its anisotropic structure and responsive behaviour to the changes in the environment. Finger pad stratum corneum (SC), the outer layer of the epidermis, is comprised of corneocytes [19] with the thickness varying between 200 and $350 \mu \mathrm{m}$ [2022]. This is much higher than the SC in other anatomical locations, where it is usually observed in the range of 10-40 $\mu \mathrm{m}$ [23-25]. While asperity height is significantly lower than stratum corneum layer thickness, microscale skin deformation and development of the real contact area is expected to depend mostly on the elastic properties of the SC. Indentation measurements to study the Young's modulus of SC are usually performed on the volar forearm skin, where hair is not present, and the skin has uniform structure with relatively low roughness. Mechanical properties of the finger pad skin are harder to evaluate due to the curvature and periodic ridge structure. Furthermore, a measured effective elastic modulus heavily depends on indenter size and skin displacement due to the underlying softer dermis layer [26,27]. Abdouni et al. [28] used a glass indenter with a curvature of $2.5 \mathrm{~mm}$ and an air blast system to measure the finger pad elastic moduli in-vivo, which was found to be in the range between 10 and $100 \mathrm{kPa}$. Similar effective elastic modulus of $70 \pm 20 \mathrm{kPa}$ was observed by P. Cornuault et al. [29]. In-vitro SC indentation measurements provide dramatically higher values in the range from 1 MPa to 1 GPa [23-25]. It remains unclear what skin elastic modulus should be used for the surface texture design and how it scales with individual asperity dimensions. 
The aim of the current work is to use a contact transition, from an asperity-only to a full contact state, as a cue to estimate skin Young's modulus at the micro-scale. The contact state is detected through friction measurements of a finger pad sliding against textured surfaces. After the point of transition, the real contact area of the skin is expected to escalate, thus leading to the increase in the observed friction coefficient. While such effect was discussed by other researchers [18,30,31], its development with normal load was not shown experimentally. At the point of transition, a combined micro displacement of the asperities and the skin equals the asperity height. Therefore, the skin elastic modulus can be calculated, if the applied forces and the counter-material properties are known. Moreover, by using multiple rubber grades with similar surface energy but various hardness, interfacial shear strength remains relatively similar, while the relative asperity displacement is varied; consequently, the asperity normal loads required for the contact transition to happen are modified. While the normal forces applied to asperities are inversely proportional to the apparent contact area, adding varying material properties to the sample matrix allows for comparison of relative changes in the fingerprint ridge contact area and the contact model accuracy improvement. Observations of the micro-contact behaviour will allow to optimise surface texture dimensions and predict finger pad friction.

\section{MATERIALS AND METHODS}

\subsection{Materials}

A total number of twelve samples were studied in this work: two surfaces with a textured area of 50x50 mm and a non-textured reference, each reproduced in a series of four various silicon rubber grades. Both textures consist of evenly distributed square packed asperities with an average asperity tip radius of $19 \pm 3 \mu \mathrm{m}$ and a height of $28 \pm 2 \mu \mathrm{m}$. The patterns differ in the asperity density created by asperity lateral spacings of 100 and $200 \mu \mathrm{m}$ (Figure 1). The surface topography of the samples was measured using a 3D LED confocal microscope S neox (Sensofar, Spain).

Negative textures were produced by laser ablation on a stainless spring steel sheet, from which textured master samples were created in a silicone 

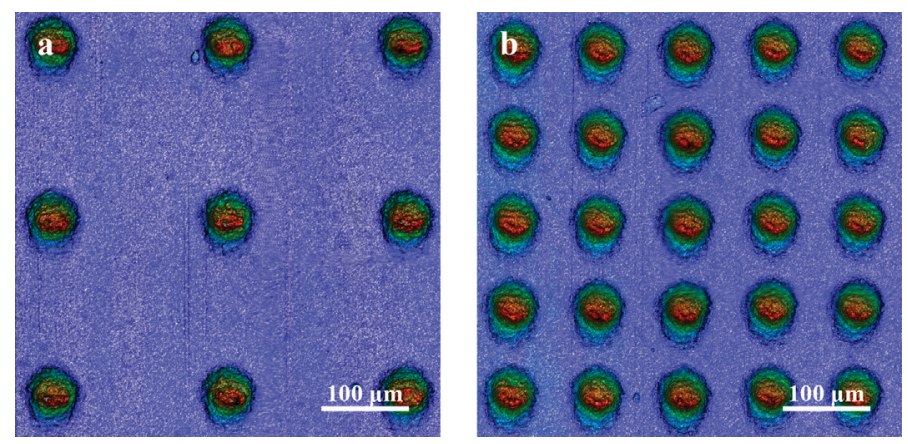

FIGURE 1 Confocal images of the produced sample textures with asperity spacing of 200 $\mu \mathrm{m}(\mathrm{a})$ and $100 \mu \mathrm{m}(\mathrm{b})$.

rubber by moulding. The master samples were replicated by a double moulding technique using polyurethane resin Smooth-Cast ${ }^{\circledR} 305$

(Smooth-on, USA) for a negative mould. This intermediate step was used to reduce the adhesion during separation and improve reproducibility of the micro-texture dimensions. The samples were moulded with constant thickness of $10 \mathrm{~mm}$ in various hardness grades using commercially available tin cure silicone rubber Mold Max $^{\mathrm{TM}}$ (Smooth-on, USA), further abbreviated by letter M followed with elastomer respective Shore A hardness. Rubber elastic moduli was estimated from the materials' technical datasheets and presented in the Table 1 along with the measured texture dimensions of the tested surfaces.

\subsection{Friction measurements}

Experiments were performed in-vivo on a dedicated setup designed by Klaassen et al. [32]. The test rig was adapted for the finger pad friction measurements and consists of an enclosed chamber with a linear reciprocating tribometer (Figure 2). The right hand is placed on a stage with an index finger inclination angle of 30 degrees relative to the sample. A transducer is mounted on the mechanical hinge and the normal load is applied with static weights. The samples were glued to polymethyl methacrylate (PMMA) plates, which were fastened to the load cell by bolt fixture. The sample textures were aligned parallel to the sliding direction. 


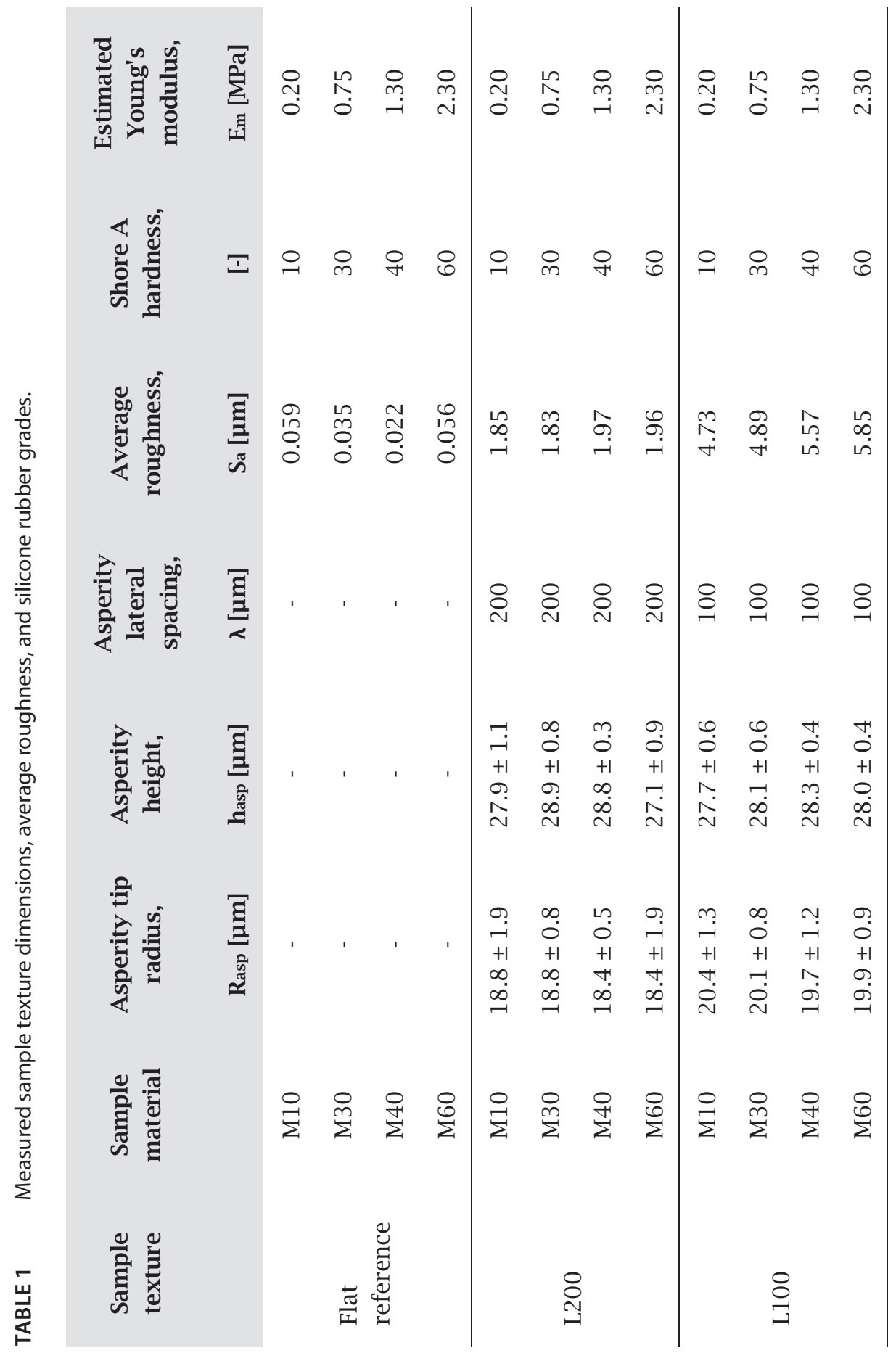




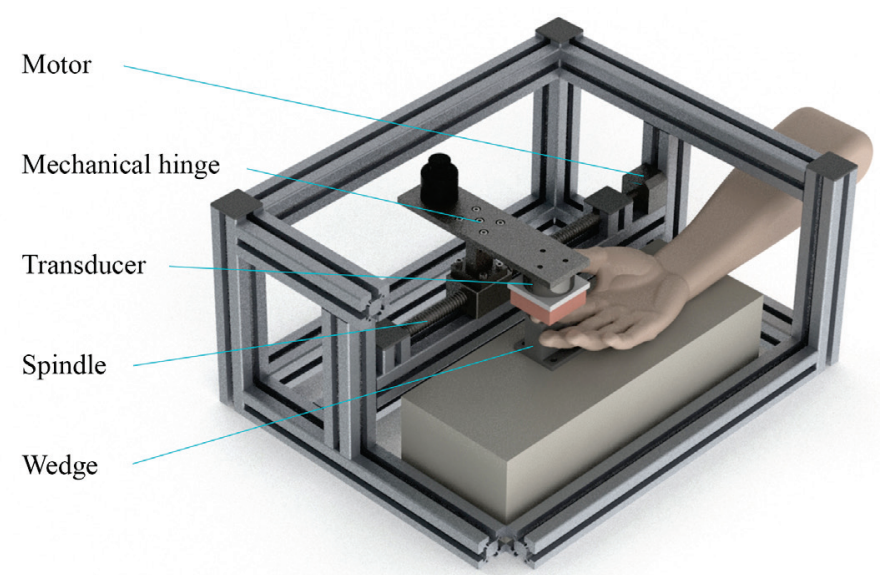

FIGURE 2 Schematic representation of the test setup used for the friction measurements.

Friction measurements were conducted with a constant sliding velocity of $2 \mathrm{~mm} / \mathrm{s}$ and a stroke length of $20 \mathrm{~mm}$. Acceleration was set to $2 \mathrm{~mm} / \mathrm{s}^{2}$ so it did not influence the dynamic coefficient of friction. The load cell was moving from the subject body, thus simulating the usually performed backward finger pad friction experiments. After each measurement the hinge was lifted, and the carriage was returned to the starting position.

The samples were tested with a large array of normal loads to detect the changes in friction coefficient with high precision. The low loads region, where adhesion friction component remains predominant, was of particular interest. Therefore, each sample was measured under 19 normal loads: from 0.1 to $0.6 \mathrm{~N}$ with a step of $0.1 \mathrm{~N}$, from 0.6 to $2 \mathrm{~N}$ with a step of $0.2 \mathrm{~N}$ and from 2 to $5 \mathrm{~N}$ with a step of $0.5 \mathrm{~N}$. Normal load was applied for 5 seconds before the motor movement was initiated. Three consecutive measurements were performed per each applied normal load.

Given the test matrix, the experimental part was split into separate measurement series and was carried out on different days. Each test series included four samples with the same representative surface but various rubber grades. The samples were tested consecutively with increasing normal load. However, the above approach means that the tests were not fully randomized - in part due to the size of the test matrix and in part due to concerns of the possible texture wear and, therefore, change of the contact conditions. This is acknowledged as a limitation of 
the work presented. That said, all environmental conditions are reported below, and their effects are considered in section 4 .

Prior to the measurements the samples were cleaned with acetonemoistened and alcohol-moistened tissues, rinsed with demineralized water and kept in a vacuum chamber for 12 hours prior to the experiment to prevent possible rubber swelling.

Experiments were carried out in ambient environmental conditions, which are presented in Table 2 . The hands were washed with soap and dried in air for 15 minutes before friction measurements against each sample. Skin hydration of the index finger pad was monitored with Corneometer CM 825 (Courage + Khazaka GmbH, Germany) to ensure that moisture content remained relatively stable between testing various sample materials. The average hydration level of the skin remained at $55 \pm 7 \mathrm{AU}$, which corresponds to the normal skin hydration level [33].

TABLE 2 Humidity and temperature during measurements.

\begin{tabular}{|c|c|c|c|c|}
\hline & $\begin{array}{c}\text { Flat } \\
\text { reference }\end{array}$ & L100 & L200 & $\begin{array}{c}\text { Fingerprint area } \\
\text { measurements }\end{array}$ \\
\hline Humidity [\%] & $63 \pm 5$ & $42 \pm 3$ & $56 \pm 4$ & 55 \\
\hline Temperature $\left[{ }^{\circ} \mathrm{C}\right]$ & $24 \pm 1$ & $23 \pm 1$ & $24.5 \pm 1$ & 23 \\
\hline
\end{tabular}

\subsection{Apparent contact area measurements}

Gross and apparent contact areas of the tested index finger pad were measured with a digital fingerprint scanner FS50 (Futronic Technology Co. Ltd., China) during sliding. Inclination angle and sliding velocity were controlled manually and were kept similar to the ones used in the friction measurements. Obtained images were filtered by histogram equalization and converted into a binary image by setting a threshold value [31,34], which is further described in the supplementary material. The total number of pixels from the binary image was multiplied by a scanner resolution to calculate apparent contact areas. Gross contact area was calculated from the best ellipse fit to the image. The images were scanned 
horizontally to detect dark to bright transition points. The obtained point arrays were used for the best oval fit function.

\subsection{Data processing}

The normal and friction forces were measured using a 6-axis Mini40 transducer (Schunk, Germany) with a sampling rate of $100 \mathrm{~Hz}$. The load cell has a resolution of $6 \mathrm{mN}$ in the normal direction and $2 \mathrm{mN}$ in the tangential direction.

In this work we differentiate the friction coefficient as measured during the whole test from the relevant dynamic friction coefficient using a Matlab code. Succinctly put, this is a stable region after the first local maximum of a high order polynomial fit (Figure 3). The reason for doing this is to ensure that the initial slope, which corresponds to the elastic deformation before relative sliding motion starts, does not affect the average values. The friction measurements performed at normal load of $0.1 \mathrm{~N}$ were filtered manually due to the high deviations in the friction coefficient curve.

Moreover, a high wavelength noise was induced to the measurements by the guide bearing, which corresponds to the pitch distance and the

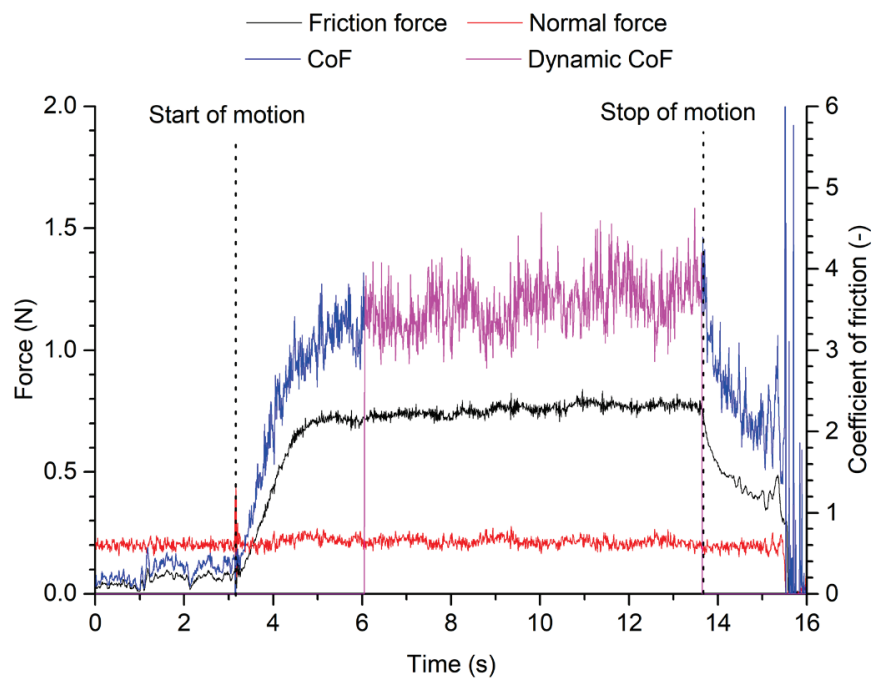

FIGURE 3 Raw force signals with calculated and extracted dynamic friction coefficient. Non-textured M60 sample at $0.2 \mathrm{~N}$ normal load. 
number of complete shaft revolutions. Therefore, the sine component with a frequency of $0.5 \mathrm{~Hz}$ was removed to reduce signal deviations, also by a Matlab script. A sine wave with a given frequency was fitted to find an amplitude and a phase offset for each measurement. Those values were used to subtract a corresponding sine function from the original signal. Inclination of the signal remained unchanged to ensure that filtering will not influence average friction values, but only reduce the standard deviation caused by induced signal noise.

\section{CALCULATION}

\subsection{Apparent contact area}

Considering a relatively soft sample material, a measured gross contact area must be corrected for the sample elasticity. Based on the Hertzian contact theory the elastic modulus of the finger pad $E_{f p}$ can be estimated from the fingerprint scanner measurements as [35]:

$$
E_{f p}=\frac{3}{4} \frac{F R_{e}}{a_{g}^{3}}\left(1-\mathrm{v}_{f p}^{2}\right)
$$

where $R_{e}$ and $v_{f p}$ is an equivalent radius and Poisson's ratio of the finger pad respectively and $a_{g}$ is an equivalent radius of the measured gross contact area. Therefore, the corrected gross contact area Agc can be described as:

$$
A_{g c}=\left(\frac{3}{4} \frac{F R_{e}}{E_{0}^{*}}\right)^{\frac{2}{3}}
$$

with a reduced elastic modulus $E_{0}^{*}=1 /\left(\left(1-v_{f p}^{2}\right) / E_{f p}+\left(1-v_{m}^{2}\right) / E_{m}\right)$, where $\boldsymbol{U}_{m}$ and $E_{m}$ are a Poisson's ratio and a Young's modulus of the tested material. Assuming that apparent to gross contact area ratio remains the same at a given normal load as measured $A_{a} / A_{g}$, the new corrected ridge contact area $A_{a c}$ can be calculated as: 


$$
A_{a c}=A_{g c} A_{a} / A_{g}
$$

The number of micro asperities in contact with the finger $N$ is calculated from the lateral spacing of the features $\lambda$ as [17]:

$$
N=A_{a c} / \lambda^{2}
$$

Therefore, the mean normal force acting on the individual asperity $\bar{F}_{i}$ can be expressed through normal load or mean apparent pressure $\bar{p}$ as:

$$
\bar{F}_{i}=F / N=\bar{p} \lambda^{2}
$$

\subsection{Pressure distribution}

The largest local skin displacement occurs in the region of maximum pressure, where local normal loads are the highest. Assuming a Hertzian spherical pressure distribution and a corresponding ratio $p_{\max } / \bar{p}$ of 1.5 , the maximum forces per asperity can be calculated as $F_{i_{\max }}=1.5 * \bar{F}_{i}$.

For the defined asperity geometry, the combined micro displacement of the skin stratum corneum layer and individual asperity will reach asperity height at an unknown force per asperity $F_{i_{c r i t}}$ and a corresponding mean apparent pressure $\bar{p}_{\text {crit }}$. It leads to the transition from asperity-only to full surface contact. With an apparent contact area equivalent radius $a_{a c}$ the part of the apparent contact area in transition can be found from Hertzian spherical pressure distribution:

$$
p(r)=\frac{3}{2} \bar{p} \sqrt{1-\frac{r^{2}}{a_{a c}^{2}}}
$$

Substituting the radii and pressure in the expression, it can be rewritten for the transition case as:

$$
F_{i_{\text {crit }}}=\frac{3}{2} \bar{F}_{l} \sqrt{1-\frac{A_{\text {trans }}}{A_{a c}}},
$$


where $A_{\text {trans }}$ is an apparent contact area in the full contact state. Therefore, the ratio of the deformed-to-apparent contact area can be calculated as:

$$
\frac{A_{\text {trans }}}{A_{a c}}=1-\left(\frac{2}{3} \frac{F_{i_{\text {crit }}}}{\bar{F}_{i}}\right)^{2}, 0 \leq \frac{A_{\text {trans }}}{A_{a c}}<1 ;
$$

Similarly, the equivalent radius of the zone in full contact $a_{\text {trans }}$ can be found as:

$$
a_{\text {trans }}=a_{a c}\left(A_{\text {trans }} / A_{a c}\right)^{1 / 2}
$$

The mean pressures in full contact and asperity contact regions, $\bar{p}_{\text {trans }}$ and $\bar{p}_{a s p}$ respectively, can be calculated through integration of the Hertzian pressure distribution equation over the corresponding areas:

$$
\begin{gathered}
\bar{p}_{\text {trans }}=\frac{1}{\pi a_{\text {trans }}^{2}} \int_{0}^{a_{\text {trans }}} \frac{3}{2} \bar{p} \sqrt{1-\frac{r^{2}}{a_{a c}^{2}}} * 2 \pi r d r \\
\bar{p}_{\text {asp }}=\frac{1}{\pi\left(a_{a c}^{2}-a_{\text {trans }}^{2}\right)} \int_{a_{\text {trans }}}^{a_{a c}} \frac{3}{2} \bar{p} \sqrt{1-\frac{r^{2}}{a_{a c}^{2}}} * 2 \pi r d r
\end{gathered}
$$

\subsection{Asperity forces}

The critical force per asperity $F_{i_{c r i t}}$ can be calculated if material properties, asperity dimensions and normal load $F_{\text {crit, }}$ at which transition starts, are known. If the Hertzian theory is applied to the micro-contact at a point when combined micro-displacement $\delta$ equals the asperity height $h_{a s p}$, the following set of equations can be written:

$$
\left\{\begin{array}{l}
F_{i_{c r i t}}=\frac{3}{2} \bar{F}_{l}=\frac{3}{2} \frac{F_{c r i t}}{A_{a c}} * \lambda^{2} \\
\delta=h_{a s p}=\left(\frac{9}{16} \frac{F_{i_{c r i t}}^{2}}{E_{1}^{* 2} R_{a s p}}\right)^{\frac{1}{3}} .
\end{array}\right.
$$


$R_{a s p}$ is the radius of an individual asperity; $E_{1}^{*}$ is a reduced Young's modulus for micro contact and equals $E_{1}^{*}=$ $1 /\left(\left(1-v_{s m c}^{2}\right) / E_{s m c}+\left(1-v_{m}^{2}\right) / E_{m}\right)$, where $v_{s m c}$ and $E_{s m c}$ are a Poisson's ratio and an effective Young's modulus of the skin in micro-scale contact respectively. Therefore, the dependency of the skin effective elastic modulus and the normal load, at which transition starts, can be expressed after the simplification as:

$$
F_{c r i t}=\frac{8}{9} \frac{\sqrt{h_{a s p}^{3} R_{a s p}}}{\lambda^{2}} * A_{a c} * E_{1}^{*}
$$

While simplified Hertzian contact can be used as a first approximation, it cannot be applied for precise calculations due to the high asperity density and contribution of the compressive stresses from the surrounding surface features. This approach overestimates the effective elastic modulus and the normal forces required for the contact transition to happen.

The problem of stress distribution at any point in a semi-infinite isotropic and homogeneous elastic half-space was solved by Boussinesq [35] and is widely used in numerical contact models [36-38]. Therefore, calculation of stresses on the micro-scale is performed by a numerical half-space contact model assuming the skin as an ideal flat elastic surface. The asperity height data obtained from confocal microscopy is used as a countersurface, thus representing microroughness which includes deviations in geometry. The simulation is performed in iterations changing the elastic skin modulus until the normal force, at which transition occurs in experiments, does not fit the normal force used in simulation. The point at which the contact transition happens is defined as the point when the total micro-displacement equals the mean asperity height. After the solution is found, $F_{i_{c r i t}}$ is calculated as the force acting on an individual asperity. The corresponding mean apparent contact pressure required for the contact transition can be found as $\bar{p}_{\text {crit }}=$ $2 / 3 * F_{i_{\text {crit }}} / \lambda^{2}$.

A graphical representation of the proposed approach is shown in Figure 4 and illustrates the dependency similar to Eq. (14). Figure 4a shows an example of calculations performed for the constant skin elastic modulus, 
surface texture L200 and two materials. Contact transition occurs when the mean apparent contact pressure reaches its critical value at the normal load $F_{\text {crit }}$. Both of these values can be obtained experimentally. In turn, the critical mean pressure $\bar{p}_{\text {crit }}$ for a given texture varies as a function of the effective elastic modulus $E_{1}^{*}$ and, if the counter material elastic modulus is known, effective skin elasticity can be estimated. Moreover, if the Young's modulus of the skin is assumed constant between the measurements, relative values of the transition force $F_{\text {crit }}$ for various materials can characterize the change in the finger pad ridge contact area. The comparison for the same skin elasticity and material, but different texture is shown on Figure $4 \mathrm{~b}$. In this case, $\bar{p}_{\text {crit }}$ varies as a function of texture dimensions and limits the maximum mean pressure for the L100 texture. Therefore, finger pad skin remains in an asperityonly contact state.

With the obtained critical force values and elastic modulus the numerical half-space simulations are performed to calculate the real contact area for the three cases: no contact transition at $F_{i}=\bar{F}_{i}$, point of transition at $F_{i}=F_{i_{c r i t}}$ and full contact, where the force acting on the asperity inside the transition zone equals $F_{i}=\bar{p}_{\text {trans }} * \lambda^{2}$ and outside $F_{i}=\bar{p}_{\text {asp }} * \lambda^{2}$ as calculated by Eqs. (11) and (12). Resulting contact areas are multiplied by the corrected apparent contact area and summed up by the ratio $A_{\text {trans }} / A_{a c}$ calculated according to Eq. (9). Schematic representation of the contact regions, average apparent pressures and asperity forces is shown Figure 5. 

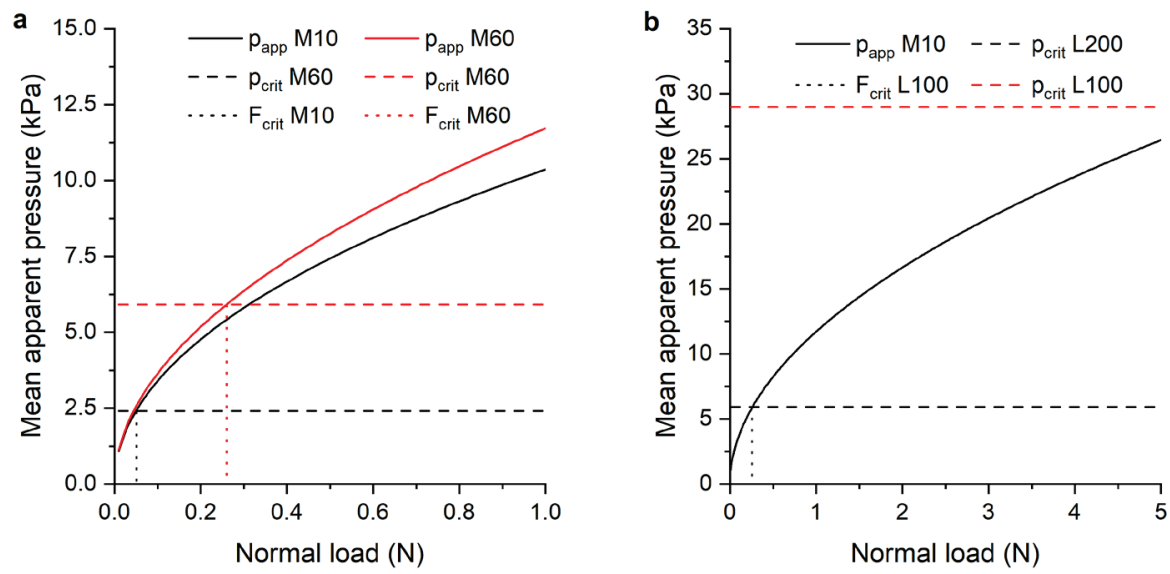

FIGURE 4 Relationship between mean apparent pressure, critical mean pressure and critical normal load for the texture L200 (a) and the material M60 (b).

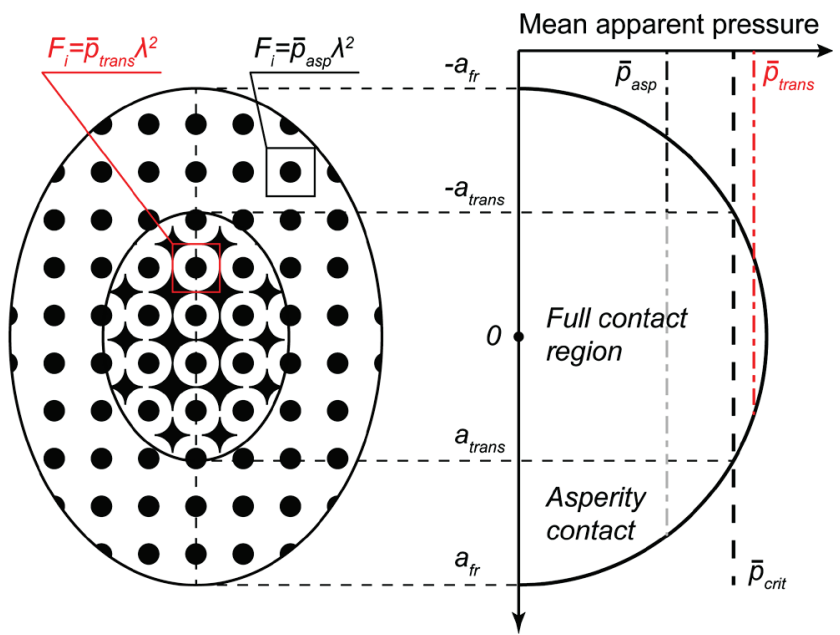

FIGURE 5 Schematic representation of the contact states, apparent mean pressures and asperity forces. 


\section{RESULTS AND DISCUSSION}

\subsection{Gross and apparent contact area}

Gross and apparent contact area measurements are shown in Figure 6 with the typical images obtained from the digital scanner presented in Figure 7. The data can be fitted to power law functions: $A_{g}=k_{g} F^{m_{g}}$ for the gross and $A_{a}=k_{a} F^{m_{a}}$ for the apparent contact area, where $k$ is a load coefficient and $m$ is a load index. Best power fits give the coefficients of 164 and 69 and the load indices of 0.16 and 0.28 for the finger pad and ridge contact areas respectively. However, the quality of such fits is relatively poor for both low and high normal loads, where the measured contact area shows lower values.

Recently, Dzidek et al. [27] explained the nature of such behaviour by the change in elastic modulus of the finger pad with displacement. At low normal loads the stiffer SC layer significantly contributes to the effective Young's modulus, but with an increase of displacement its influence reduces, and elastic modulus becomes governed by the dermis. At high normal loads the elastic modulus of a finger pad rises again as it becomes compressed against a distal phalanx bone. At this point further contact area development becomes constrained by the skin stress-strain behaviour.

Liu et al. [39] reported similar observations and proposed a two term function which separates two normal load regions. Following their approach, gross contact area fit develops with coefficients of 159 and 171 and exponents of 0.22 and 0.12 for low and high normal load regions respectively. The apparent contact area increases slightly faster, with the corresponding coefficients of 64 and 73 and exponents of 0.42 and 0.23 , which leads to the gradual increase of the ridge to gross contact ratio up to 0.52 at $5 \mathrm{~N}$. 

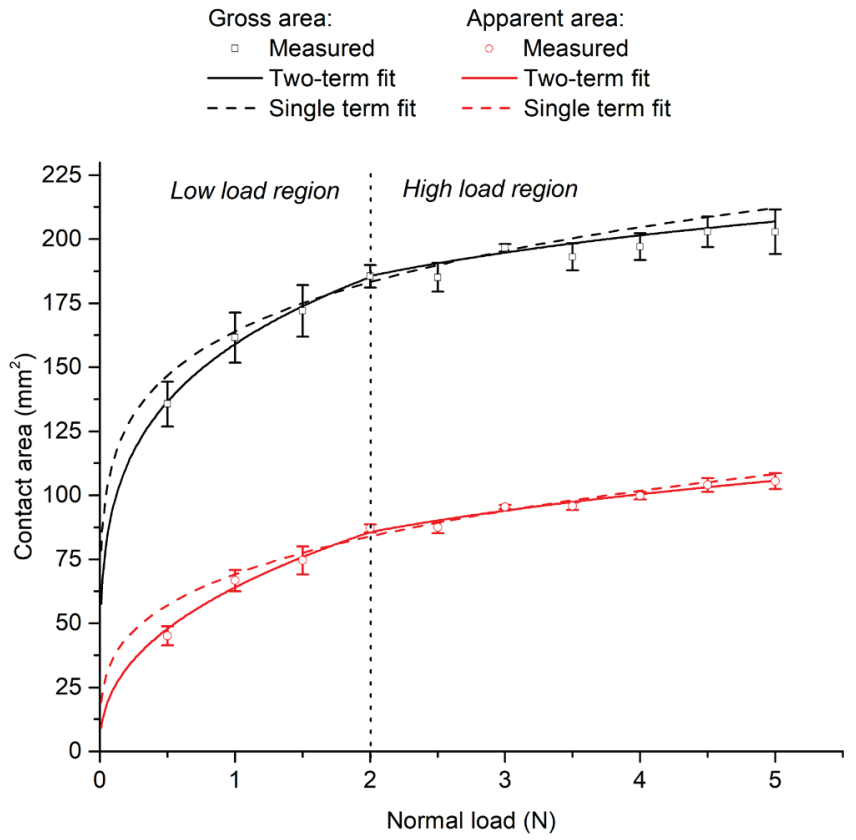

FIGURE $6 \quad$ Experimental gross and apparent contact areas.
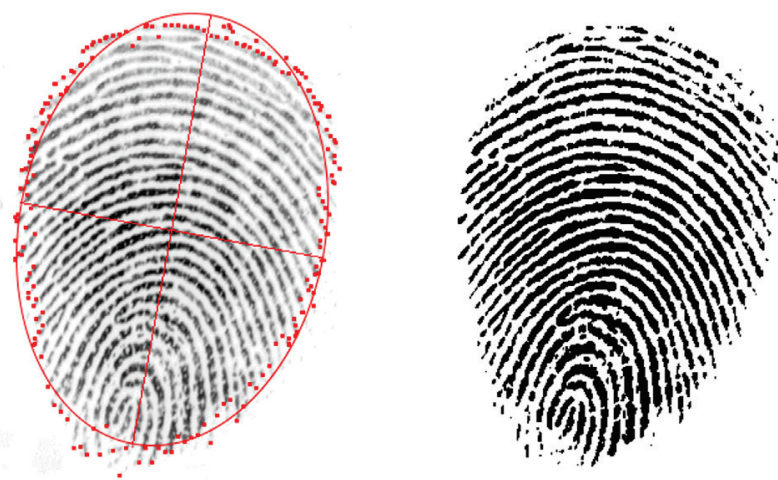

FIGURE $7 \quad$ Typical example of the finger pad elliptic fit and apparent contact area after image filtering at $2 \mathrm{~N}$ normal load. 
Reported exponents for the apparent contact area of the index finger pad in literature vary significantly from 0.2 to 0.7 [27,39-41] and depend on the finger pad inclination angle, environmental conditions and personal differences. Moreover, the calculated coefficients are influenced by the image processing and selected threshold for the ink print or digital scanner measurement methods. Therefore, absolute values cannot be compared with other reports.

\subsection{Texture and sample hardness influence}

Figure 8 shows calculated friction coefficients for the four materials tested. For the smooth reference surfaces, the coefficient of friction reduces with an increase of the normal load and follows a commonly observed power law function: $\mu \propto F^{n-1}$, with the $n$ of $0.75,0.67,0.58$ and 0.79 for all the rubber grades from the softest to the hardest respectively. For an elastic material the exponent $n$ shall decrease with the rise in the rubber Young's modulus due to the reduction in contact area and hysteresis friction component. However, the results for the M60 sample show a contradicting behaviour.

Moreover, the hardest non-textured sample shows a distinctive local increase in friction at $1.8 \mathrm{~N}$, which breaks the consistent power trend and reduces the coefficient of determination. Small deviations for other reference samples can also be observed in the region between 1 and $2 \mathrm{~N}$. The contacting surfaces are not ideally smooth, and their contact compliance is directly related to the material hardness. Therefore, the observed local peaks are possibly connected to the deformation of the asperities and transition to the full contact state. It implies that the power function fitted to the hardest reference sample cannot be used to estimate the apparent contact area development.

All L200 textures show a completely different trend as compared to the reference samples. At low normal loads the coefficient of friction is significantly reduced, but it rises quickly with a further increase of normal load, coming to its maximum around $0.8 \mathrm{~N}$. This peak is followed by a steady decline with the values nearly reaching the friction results of the non-textured samples. Similar bell-curved shapes of the friction coefficient were reported previously as the influence of moisture [22,42,43]. Tomlinson et al. [42] concluded that an increase in friction comes mainly from the rise in the real contact area as skin softens. 
a

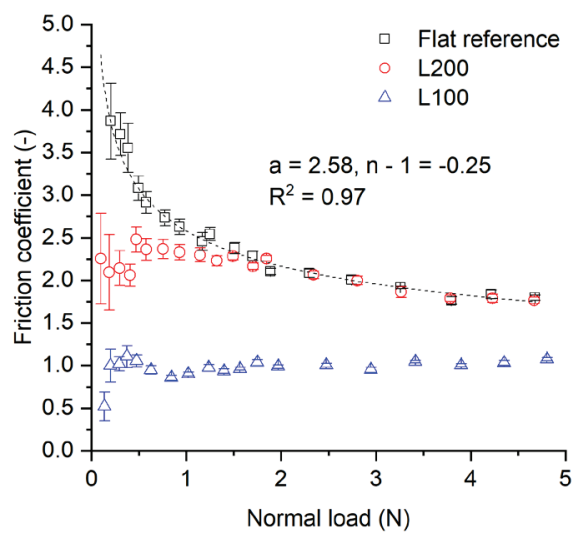

C

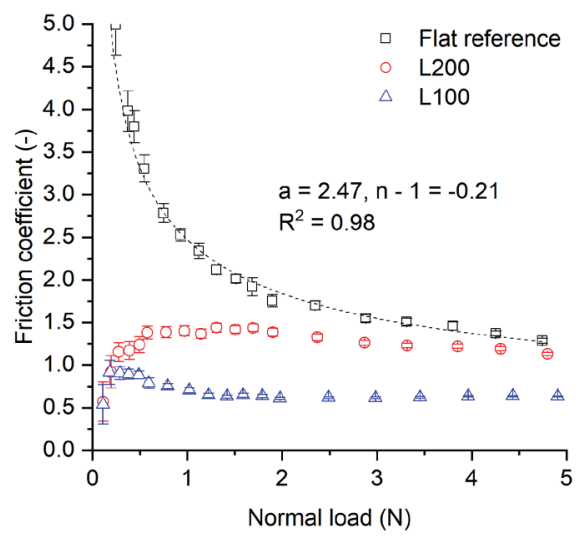

b

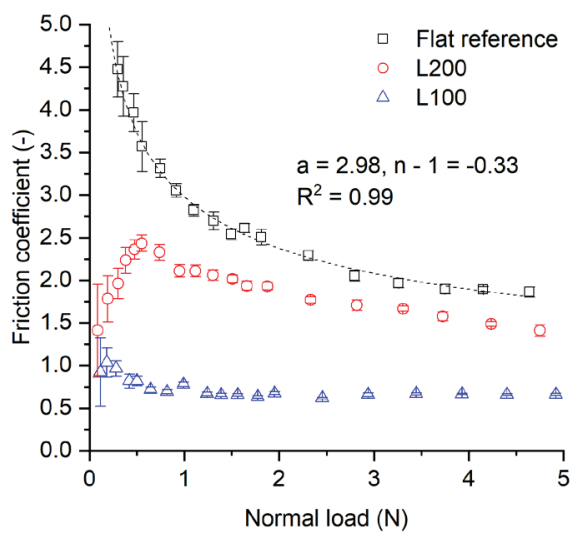

d

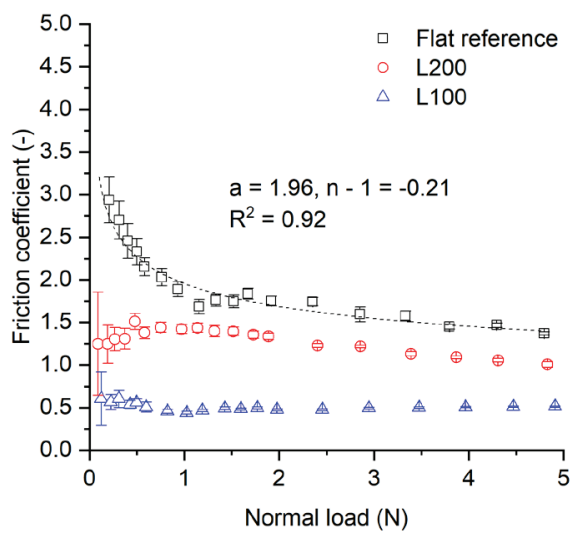

FIGURE 8 Calculated friction coefficients grouped by rubber grades: M10 (a), M30 (b), M40 (c), M60 (d).

Three contact cases can be considered for the skin sliding against textured surfaces. Initially the skin is supported only by the texture asperities, which reduce the real contact area. With an increase of the normal load, a combined micro displacement of skin and asperities will reach the asperity height value, leading to the contact transition state. This case can be characterized by a rapid growth of the real contact area and therefore drop in the mean pressure. Once the contact stabilizes and reaches a full contact state, the mean pressure starts to gradually rise again. 


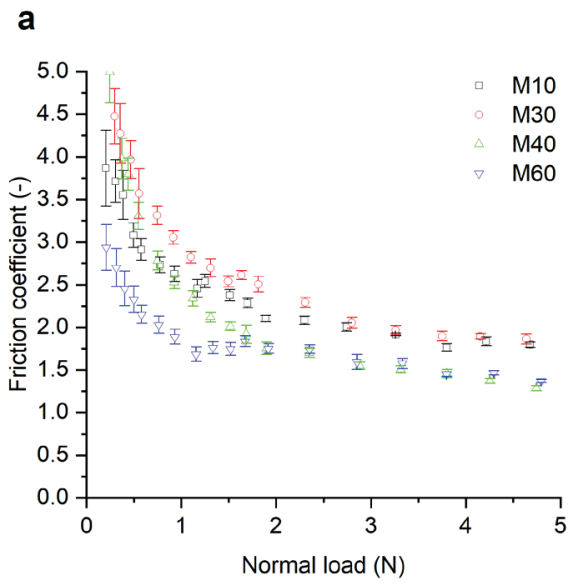

b

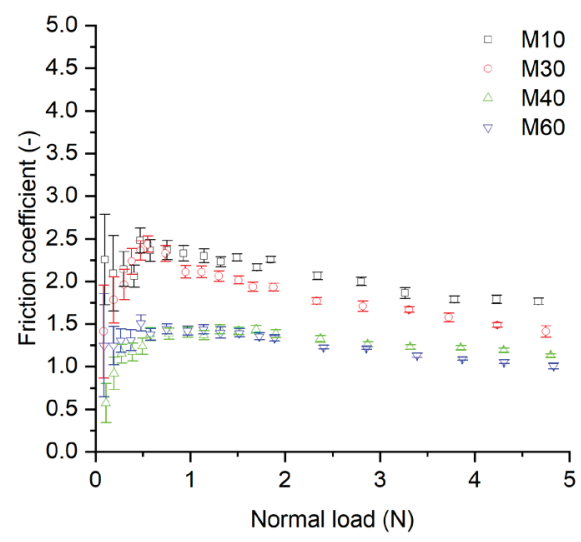

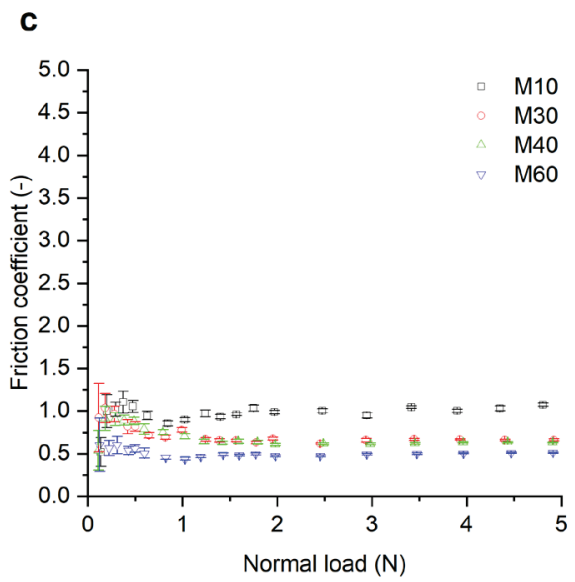

FIGURE 9 Calculated friction coefficient grouped by surface texture: flat reference (a), L200 (b) and L100 (c).

The high density L100 texture shows significantly lower values of the friction coefficient (between 0.5 and 1), which remain almost constant for the entire range of the tested normal loads. Only the softest high-density texture demonstrates a linear increase in the coefficient of friction, which can be connected to the predominant deformation of the texture asperities and higher hysteresis friction losses due to the larger material displacement [44].

A slight increase in the friction coefficient can be observed for all L100 textures at the normal loads around 0.3-0.5 N. It diminishes with an 
increase in sample hardness and can be explained by material deformation. As discussed earlier, the finger pad Young's modulus reduces with displacement at low normal loads [27]. Consequently, the initial increase in gross and apparent contact area on a soft material is achieved mainly by its deformation, while finger pad displacement remains relatively low.

With an increase in material hardness, the coefficient of friction generally decreases (Figure 9). The observed deviation in the relative friction values for the M30 reference sample in Figure 9a can be explained by the lower surface roughness. Small variations of the relative friction values are assumed to arise from the minor changes in stratum corneum hydration level during a given measurement series.

\subsection{Modelling results}

Friction can be approximated by the two-term model expression (1), where the adhesion term can be described as a product of interfacial shear strength and real contact area $F_{a d h}=\tau A_{r}$. Adams et al. [7] proposed that interfacial shear strength can be expressed as $\tau=\tau_{0}+\alpha \bar{p}_{r}$ accounting for the organic film in the dry contact, where $\tau_{0}$ is an intrinsic shear strength, $\alpha$ is a pressure coefficient and $\bar{p}_{r}$ is a mean real pressure. A hysteresis component arising from an elastic material deformation can be described as [44]:

$$
F_{\text {def }}=\beta\left(\frac{9}{128 R_{e}}\right)^{\frac{2}{3}}\left(\frac{F^{4}}{E_{0}^{*}}\right)^{\frac{1}{3}}
$$

where $B$ is viscoelastic hysteresis loss fraction, $R_{e}$ is the equivalent finger pad radius, $E_{0}{ }^{*}$ is the effective Young's modulus in macro contact and $F$ is applied normal load. Deformation of the skin and asperities on the microscale is assumed to be insignificant, considering low asperity heights. Therefore, the total coefficient of friction can be estimated as:

$$
\mu_{t o t}=\mu_{a d h}+\mu_{d e f}=\frac{\tau_{0} A_{r}}{F}+\alpha+\beta\left(\frac{9}{128 R_{e}}\right)^{\frac{2}{3}}\left(\frac{F}{E_{0}^{*}}\right)^{\frac{1}{3}}
$$


The approach described in Section 3 was used to estimate an effective elastic modulus of the skin at the micro-scale and to calculate real contact areas. Finger pad equivalent radius $R_{e}$ was estimated as $11 \mathrm{~mm}$, while Poisson's ratios for the skin and silicone rubber grades were assumed to be equal 0.5 and 0.49 respectively $[5,45,46]$. With the curve fits of the apparent contact area obtained from a fingerprint scanner the elastic modulus in the range of $0.2-0.5 \mathrm{MPa}$ was calculated (Table 3) Nonetheless, the same relative position of the inverse pressure peaks at the observed normal load of $0.8 \mathrm{~N}$ cannot be obtained. Furthermore, the measured exponents of 0.42 and 0.23 are too low compared to the power of 0.58 obtained from the experiment.

TABLE 3 Calculated Young's modulus of the skin at the micro-scale and microdeformations at the point of transition for the L200 texture.

\begin{tabular}{lcccc}
\multicolumn{1}{c}{ Material } & M10 & M30 & M40 & M60 \\
\hline Calculated Young's modulus [MPa] & 0.5 & 0.2 & 0.25 & 0.2 \\
SC deformation $[\mu \mathrm{m}]$ & 8.7 & 22.9 & 25.4 & 29.1 \\
Asperity deformation $[\mu \mathrm{m}]$ & 21.2 & 6.1 & 4.8 & 2.5
\end{tabular}

Clearly, the simple approximations and assumptions described by Eqs. (3) and (4) cannot be applied to estimate gross and apparent contact areas for elastomers. Experimental observations suggest that finger pad elastic modulus should be considered as a function of displacement and gross contact area [27,39]. Compared to the rigid materials, gross contact area with elastomers will develop faster, thus increasing the depth of the maximum subsurface stresses as described by Hertzian contact. It will reduce the influence of stratum corneum on finger pad Young's modulus at low normal loads. The constraint arising from distal phalanx bone and skin strain will also be less pronounced, due to the reduced relative deformation of the finger pad and higher rubber compliance. Therefore, with the reduction of the counter-material elastic modulus, the contact area development trend shown in Figure 6 will change at greater normal load and the two-term evolution can take the form of the one-term power law fit. 
a

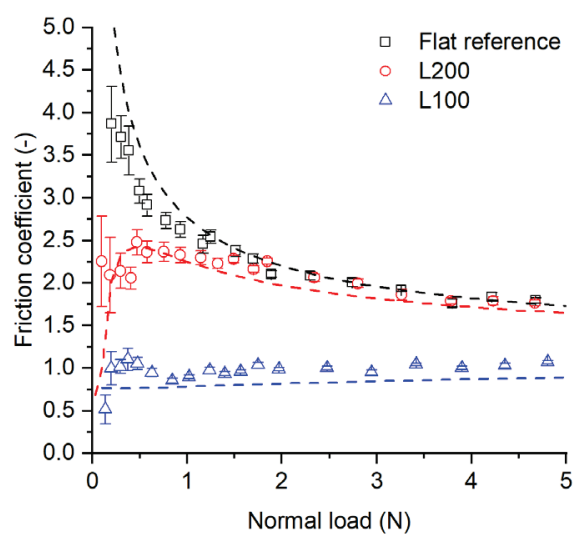

C

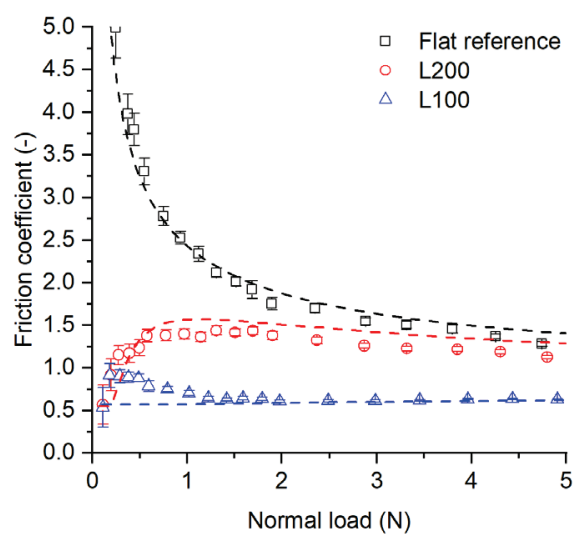

b

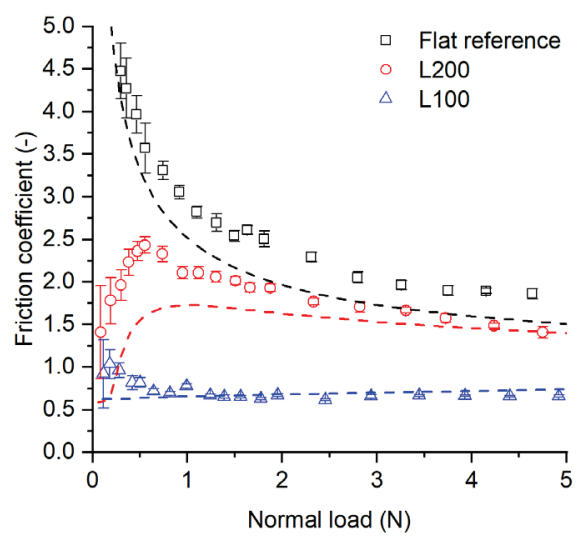

d

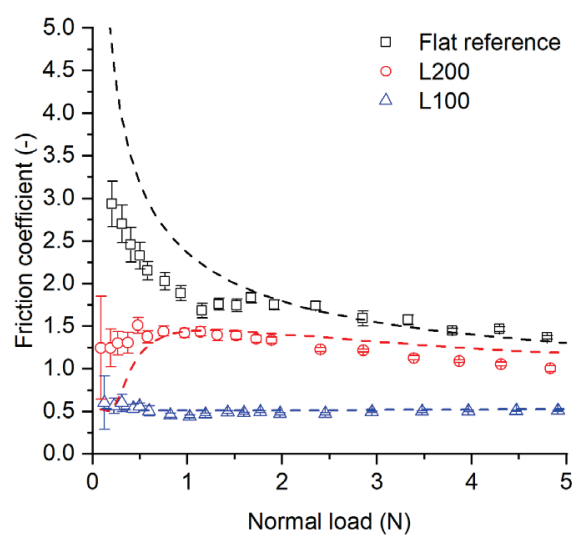

FIGURE 10 Closest model fit for the friction coefficient measurements grouped by rubber grades: M10 (a), M30 (b), M40 (c), M60 (d).

The obtained elastic modulus is of the same order and comparable to optical coherence tomography measurements and values calculated from the fingerprint ridge deformations $[27,46]$. However, the calculated values are significantly smaller than typically reported from in-vitro indentation measurements, where the values vary from 0.6 MPa to 1 GPa [23,25]. Pailler-Mattei et al. [23] reported the reduction of the SC Young's modulus from $1 \mathrm{GPa}$ to $1 \mathrm{MPa}$ with an increase of the penetration depth from 10 $\mathrm{nm}$ to about $4 \mu \mathrm{m}$ on isolated skin samples. If the stratum corneum elasticity continues to decrease with further deformation, the calculated values become feasible, considering, that the indentation depths in the 
current work are much higher (see Table 3). Moreover, it can provide another possible explanation for the higher Young's modulus obtained for the M10 sample. Such behaviour can be explained by anisotropic SC structure and increase of its moisture content with depth, which makes the skin more compliant at higher displacements.

The closest model fit was found for the $A_{a} \approx 85 F^{0.49}$ with corresponding skin elastic modulus of $0.2 \mathrm{MPa}$ for L200 and reference samples and with $0.4 \mathrm{MPa}$ for L100 texture. Such an exponent for the apparent contact area seems reasonable considering the lowest experimental friction coefficient exponent $n-1=-0.42$. While it does not allow for analysing of the results quantitatively, the relative values still explain the observed friction behaviour, as the real contact area remains directly proportional to the apparent contact area. The intrinsic interfacial shear strength $\tau_{0}$ was considered constant for all the tested materials, while the hysteresis coefficient was varied. The curve fits were obtained with a pressure coefficient $\alpha=0.35$ and presented in Figure 10. While the friction coefficient can be fitted relatively well, it is impossible to achieve a good result in both low and high normal load regions for various materials by using a single term power law.

\subsection{Surface wear}

Surface wear observed on the tested surfaces did not exceed the standard deviation of the texture dimensions. The only exception was for the L200 M60 samples, where approximately 35\% of the asperities were damaged by tear. An example wear profile of such an asperity is presented in Figure 11 . While the wear can be considered gradual and the full contact is achieved at relatively low normal loads, influence of wear on friction measurements is considered insignificant. According to material specification the tensile strength for the M60 is $2.7 \mathrm{MPa}$, which is the lowest among other materials. The analysis of the stress distribution could allow to estimate shear and tensile forces acting on asperity, however, it is beyond the scope of the current work. 


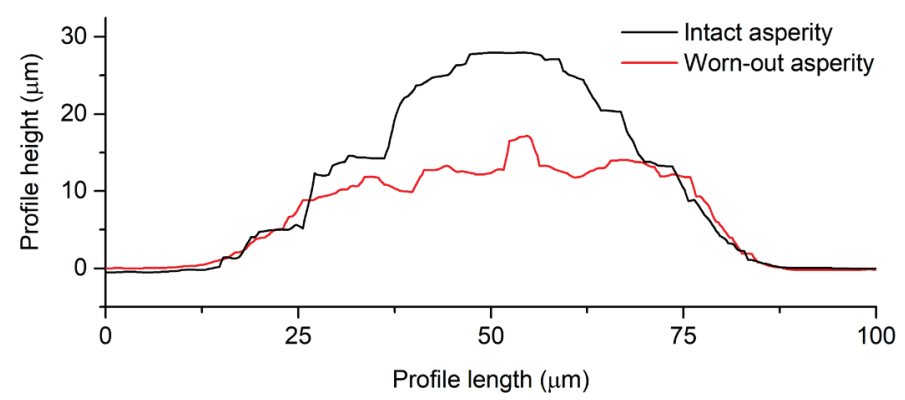

FIGURE 11 Asperity surface profile wear for the L200 M60 sample.

\section{CONCLUSIONS}

Transition from asperity-only to full contact was shown through the friction measurements. The distinctive contact transition peak is expected to become less prominent with increase of sample or skin elastic modulus, reduction of texture spacing and rise in sub-texture roughness.

The proposed method can be used for the rough estimation of skin elasticity, real contact area and texture design, if the apparent contact area development is accurately predicted. The model is limited to dry finger pad contact and restricted to the textures with lateral spacings below the fingerprint ridge width and relatively small heights as compared to the thickness of the stratum corneum.

The values of the effective skin Young's modulus estimated with the proposed empirical approach vary between 0.2 and $0.5 \mathrm{MPa}$. The obtained results are slightly higher but of the same order as values calculated from fingerprint ridge deformation models and more direct optical coherence tomography measurements.

Experimental results with elastomers show significantly higher exponents for the apparent contact area development with increase of normal load than estimated by Hertzian contact. It is expected that high countersurface compliance reduces the influence of the constraints arising from stratum corneum and distal phalanx bone at low and high normal loads respectively. 


\section{Ethical approval}

All procedures performed in the current studies involving human participant were in accordance with the ethical standards of the institutional and/or national research committee and with the 1964 Helsinki declaration and its later amendments or comparable ethical standards. The subject provided a written informed consent. None of the experiments were invasive or harmful physiologically or psychologically.

\section{Acknowledgements}

This work was supported by INTERREG V-A Deutschland - Nederland program MOVERO under the project number 142091.

\section{REFERENCES}

[1] T. Yoshioka, S.J. Bensmaïa, J.C. Craig, S.S. Hsiao, Texture perception through direct and indirect touch: An analysis of perceptual space for tactile textures in two modes of exploration, Somatosens. Mot. Res. 24 (2007) 53-70. doi:10.1080/08990220701318163.

[2] L. Skedung, K. Danerlöv, U. Olofsson, C. Michael Johannesson, M. Aikala, J. Kettle, M. Arvidsson, B. Berglund, M.W. Rutland, Tactile perception: Finger friction, surface roughness and perceived coarseness, Tribol. Int. 44 (2011) 505-512. doi:10.1016/j.triboint.2010.04.010.

[3] L.E.M. Grierson, H. Carnahan, Manual exploration and the perception of slipperiness, Percept. Psychophys. 68 (2006) 1070-1081. doi:10.3758/BF03193710.

[4] R. Fagiani, F. Massi, E. Chatelet, Y. Berthier, A. Akay, Tactile perception by friction induced vibrations, Tribol. Int. 44 (2011) 1100-1110. doi:10.1016/j.triboint.2011.03.019.

[5] R. Fagiani, M. Barbieri, A contact mechanics interpretation of the duplex theory of tactile texture perception, Tribol. Int. 101 (2016) 49-58. doi:10.1016/j.triboint.2016.03.031.

[6] G. Robles-De-La-Torre, V. Hayward, Force can overcome object geometry in the perception of shape through active touch, Nature. 412 (2001) 445-448. doi:10.1038/35086588.

[7] M.J. Adams, B.J. Briscoe, S.A. Johnson, Friction and lubrication of human skin, Tribol. Lett. 26 (2007) 239-253. doi:10.1007/s11249-007-9206-0.

[8] S.A. Johnson, D.M. Gorman, M.J. Adams, B.J. Briscoe, The friction and lubrication of human stratum corneum., Tribol. Ser. 25 (1993) 663-672. doi:10.1016/S0167-8922(08)70419-X.

[9] S. Derler, L.C. Gerhardt, A. Lenz, E. Bertaux, M. Hadad, Friction of human 
skin against smooth and rough glass as a function of the contact pressure, Tribol. Int. 42 (2009) 1565-1574. doi:10.1016/j.triboint.2008.11.009.

[10] K. Duvefelt, U. Olofsson, C.M. Johannesson, L. Skedung, Model for contact between finger and sinusoidal plane to evaluate adhesion and deformation component of friction, Tribol. Int. 96 (2016) 389-394. doi:10.1016/j.triboint.2014.12.020.

[11] S. Derler, G.M. Rotaru, Stick-slip phenomena in the friction of human skin, Wear. 301 (2013) 324-329. doi:10.1016/j.wear.2012.11.030.

[12] S.E. Tomlinson, R. Lewis, M.J. Carré, The effect of normal force and roughness on friction in human finger contact, Wear. 267 (2009) 13111318. doi:10.1016/j.wear.2008.12.084.

[13] S.E. Tomlinson, M.J. Carré, R. Lewis, S.E. Franklin, Human finger contact with small, triangular ridged surfaces, Wear. 271 (2011) 2346-2353. doi:10.1016/j.wear.2010.12.055.

[14] M.A. Darden, C.J. Schwartz, Skin tribology phenomena associated with reading braille print: The influence of cell patterns and skin behavior on coefficient of friction, Wear. 332-333 (2015) 734-741. doi:10.1016/j.wear.2014.12.053.

[15] S. Zhang, X. Zeng, D.T.A. Matthews, A. Igartua, E. Rodriguez-Vidal, J.C. Fortes, E. van der Heide, Texture design for light touch perception, Biosurface and Biotribology. 3 (2017) 25-34. doi:10.1016/j.bsbt.2017.02.002.

[16] M.A. Masen, A systems based experimental approach to tactile friction, J. Mech. Behav. Biomed. Mater. 4 (2011) 1620-1626. doi:10.1016/j.jmbbm.2011.04.007.

[17] J. Van Kuilenburg, M.A. Masen, M.N.W. Groenendijk, V. Bana, E. van der Heide, An experimental study on the relation between surface texture and tactile friction, Tribol. Int. 48 (2012) 15-21. doi:10.1016/j.triboint.2011.06.003.

[18] J. Van Kuilenburg, M.A. Masen, E. van der Heide, The role of the skin microrelief in the contact behaviour of human skin: Contact between the human finger and regular surface textures, Tribol. Int. 65 (2013) 81-90. doi:10.1016/j.triboint.2012.11.024.

[19] C. Merle, A. Baillet-Guffroy, Physical and chemical perturbations of the supramolecular organization of the stratum corneum lipids: In vitro to ex vivo study, Biochim. Biophys. Acta - Biomembr. 1788 (2009) 1092-1098. doi:10.1016/j.bbamem.2009.02.010.

[20] H. Fruhstorfer, U. Abel, C.D. Garthe, A. Knüttel, Thickness of the stratum corneum of the volar fingertips, Clin. Anat. 13 (2000) 429-433. doi:10.1002/1098-2353(2000)13:6<429::AID-CA6>3.0.CO;2-5.

[21] M.J. Adams, S.A. Johnson, P. Lefèvre, V. Lévesque, V. Hayward, T. André, J.L.L. Thonnard, Finger pad friction and its role in grip and touch, J. R. Soc. Interface. 10 (2013) 20120467. doi:10.1098/rsif.2012.0467.

[22] X. Liu, Z. Lu, R. Lewis, M.J. Carré, S.J. Matcher, Feasibility of using optical 
coherence tomography to study the influence of skin structure on finger friction, Tribol. Int. 63 (2013) 34-44. doi:10.1016/j.triboint.2012.08.020.

[23] C. Pailler-Mattei, S. Pavan, R. Vargiolu, F. Pirot, F. Falson, H. Zahouani, Contribution of stratum corneum in determining bio-tribological properties of the human skin, Wear. 263 (2007) 1038-1043. doi:10.1016/j.wear.2007.01.128.

[24] Y. Yuan, R. Verma, Measuring microelastic properties of stratum corneum, Colloids Surfaces B Biointerfaces. 48 (2006) 6-12. doi:10.1016/j.colsurfb.2005.12.013.

[25] M. Geerligs, L. van Breemen, G. Peters, P. Ackermans, F. Baaijens, C. Oomens, In vitro indentation to determine the mechanical properties of epidermis, J. Biomech. 44 (2011) 1176-1181. doi:10.1016/j.jbiomech.2011.01.015.

[26] J. van Kuilenburg, M.A. Masen, E. van der Heide, Contact modelling of human skin: What value to use for the modulus of elasticity?, Proc. Inst. Mech. Eng. Part J J. Eng. Tribol. 227 (2012) 349-361. doi:10.1177/1350650112463307.

[27] B.M. Dzidek, M.J. Adams, J.W. Andrews, Z. Zhang, S.A. Johnson, Contact mechanics of the human finger pad under compressive loads., J. R. Soc. Interface. 14 (2017) 20160935. doi:10.1098/rsif.2016.0935.

[28] A. Abdouni, M. Djaghloul, C. Thieulin, R. Vargiolu, C. Pailler-Mattei, H. Zahouani, Biophysical properties of the human finger for touch comprehension: Influences of ageing and gender, R. Soc. Open Sci. 4 (2017). doi:10.1098/rsos.170321.

[29] P. Cornuault, L. Carpentier, M. Bueno, J. Cote, G. Monteil, Influence of physico-chemical, mechanical and morphological fingerpad properties on the frictional distinction of sticky/slippery surfaces, J. R. Soc. Interface. 12 (2015) 20150495. doi:10.1098/rsif.2015.0495.

[30] S. Zhang, A. Rodriguez Urribarri, M. Morales Hurtado, X. Zeng, E. van der Heide, The role of the sliding direction against a grooved channel texture on tool steel: An experimental study on tactile friction, Int. J. Solids Struct. 56 (2015) 53-61. doi:10.1016/j.ijsolstr.2014.12.005.

[31] A.C. Rodríguez Urribarrí, E. van der Heide, X. Zeng, M.B. de Rooij, Modelling the static contact between a fingertip and a rigid wavy surface, Tribol. Int. 102 (2016) 114-124. doi:10.1016/j.triboint.2016.05.028.

[32] M. Klaassen, D.J. Schipper, M.A. Masen, Influence of the relative humidity and the temperature on the in-vivo friction behaviour of human skin, Biotribology. 6 (2016) 21-28. doi:10.1016/j.biotri.2016.03.003.

[33] U. Heinrich, U. Koop, M.C. Leneveu-Duchemin, K. Osterrieder, S. Bielfeldt, C. Chkarnat, J. Degwert, D. Häntschel, S. Jaspers, H.P. Nissen, M. Rohr, G. Schneider, H. Tronnier, Multicentre comparison of skin hydration in terms of physical-, physiological- and product-dependent parameters by the capacitive method (Corneometer CM 825), Int. J. Cosmet. Sci. 25 (2003) 4553. doi:10.1046/j.1467-2494.2003.00172.x.

[34] L.R. Manfredi, H.P. Saal, K.J. Brown, M.C. Zielinski, J.F. Dammann, V.S. Polashock, S.J. Bensmaia, Natural scenes in tactile texture, J. Neurophysiol. 
111 (2014) 1792-1802. doi:10.1152/jn.00680.2013.

[35] K.L. Johnson, Contact Mechanics, Cambridge University Press, Cambridge, 1985.

[36] G.K. Nikas, Boussinesq-Cerruti functions and a simple technique for substantial acceleration of subsurface stress computations in elastic halfspaces, Proc. Inst. Mech. Eng. Part J J. Eng. Tribol. 220 (2006) 19-28. doi:10.1243/13506501JET125.

[37] W.W. Chen, Q.J. Wang, A Numerical Static Friction Model for Spherical Contacts of Rough Surfaces, Influence of Load, Material, and Roughness, J. Tribol. 131 (2009) 021402. doi:10.1115/1.3063814.

[38] J. Cesbron, H.P. Yin, F. Anfosso-Lédée, D. Duhamel, D. Le Houédec, Z.Q. Feng, Numerical and experimental study of multi-contact on an elastic halfspace, Int. J. Mech. Sci. 51 (2009) 33-40. doi:10.1016/j.ijmecsci.2008.11.003.

[39] X. Liu, M.J. Carré, Q. Zhang, Z. Lu, S.J. Matcher, R. Lewis, Measuring contact area in a sliding human finger-pad contact, Ski. Res. Technol. 24 (2018) 3144. doi:10.1111/srt.12387.

[40] T. Soneda, K. Nakano, Investigation of vibrotactile sensation of human fingerpads by observation of contact zones, Tribol. Int. 43 (2010) 210-217. doi:10.1016/j.triboint.2009.05.016.

[41] H.T. Lin, T.F. Hong, W.L. Li, Grip performance affected by water-induced wrinkling of fingers, Tribol. Lett. 58 (2015) 1-9. doi:10.1007/s11249-0150515-4.

[42] S.E. Tomlinson, R. Lewis, X. Liu, C. Texier, M.J. Carré, Understanding the friction mechanisms between the human finger and flat contacting surfaces in moist conditions, Tribol. Lett. 41 (2011) 283-294. doi:10.1007/s11249010-9709-y.

[43] T. André, P. Lefèvre, J.L. Thonnard, A continuous measure of fingertip friction during precision grip, J. Neurosci. Methods. 179 (2009) 224-229. doi:10.1016/j.jneumeth.2009.01.031.

[44] J.A. Greenwood, H. Minshall, D. Tabor, Hysteresis Losses in Rolling and Sliding Friction, Proc. R. Soc. A Math. Phys. Eng. Sci. 259 (1961) 480-507. doi:10.1098/rspa.1961.0004.

[45] J. Ilg, S.J. Rupitsch, A. Sutor, R. Lerch, Determination of dynamic material properties of silicone rubber using one-point measurements and finite element simulations, IEEE Trans. Instrum. Meas. 61 (2012) 3031-3038. doi:10.1109/TIM.2012.2203449.

[46] X. Liang, S.A. Boppart, Biomechanical properties of in vivo human skin from dynamic optical coherence elastography, IEEE Trans. Biomed. Eng. 57 (2010) 953-959. doi:10.1109/TBME.2009.2033464. 


\section{APPENDIX A}

\section{Data processing of fingerprint images and threshold influence}

Apparent contact area was measured with a digital fingerprint scanner to account for the deformation of the ridges and rise of apparent to gross contact area ratio with increase of normal load. The scanner sensor used in this work acquires an 8-bit grayscale image and has $40.64 \times 38.10 \mathrm{~mm}$ scanning area with a resolution of $800 \times 750$ pixels respectively.

Sensor noise level was measured as the highest pixel value when no finger contact was present and equalled 15 , which is $6 \%$ of the 8 -bit scale. This threshold was used as a high-pass filter during the contact area measurements to ensure that pixel value readings are exclusively related to the finger pad contact. Image processing was performed in NI LabVIEW. Prior to further analysis, all acquired images were cropped to a rectangular region of interest of 340x420 pixels.

Histogram function varies with increase of the normal load. Therefore, it is not possible to apply a single threshold value for data evaluation. To overcome this difficulty, obtained histograms were equalized on the value range from 16 to 255. This linearizes cumulative distribution functions and preserves the total measured pixel count (Figure A.1).

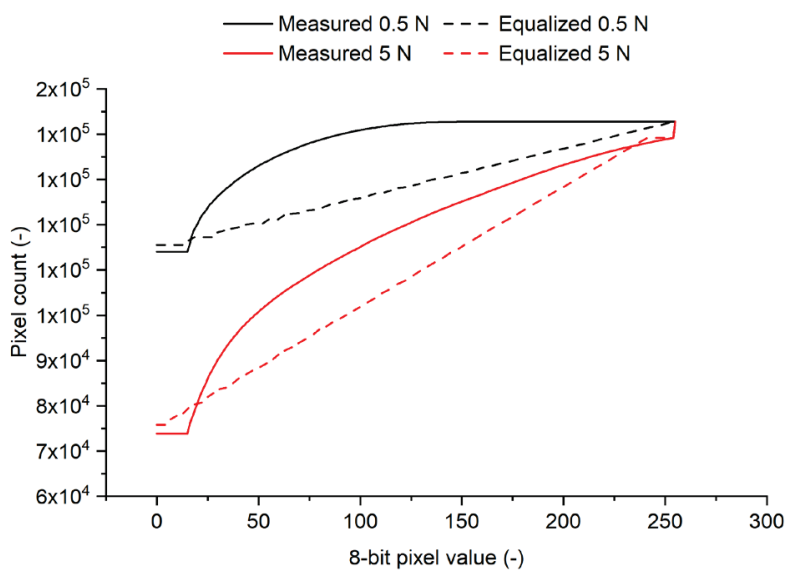

FIGURE A.1 Comparison of cumulative distribution functions before and after histogram equalization at 0.5 and $5 \mathrm{~N}$ normal loads. 
The linear cumulative function can be expressed as $y=a x / 255+b$, where $a$ is a number of pixels related to the apparent contact area and $b$ is the number of background pixels, so that the total pixel count equals $a+b$. The apparent fingerprint contact area can be calculated from a number of pixels $n$ with values higher than a selected threshold $x_{t h}$ as: $n=a+b-$ $a * x_{t h} / 255-b=a\left(1-x_{t h} / 255\right)$. Therefore, histogram equalization allows the use of a single threshold for the image processing, while cumulative pixel count remains directly proportional to the total number of measured points.

Calculation of the apparent contact area requires selection of the single threshold for the image data set with further conversion to binary. Minimum and maximum possible thresholds were selected based on false positive and false negative result cases and equalled 110 and 140 respectively. At a low threshold, false positive errors lead to "bridging" between fingerprint ridge sections, starting from the centre of an image (Figure A.2a). If the threshold is too high, it results in false negative errors and fails to detect fingerprint ridge sections (Figure A.2b).

Based on the boundary cases, an average threshold value of 125 was selected for the apparent area calculation in this study. With an apparent contact area directly proportional to the measured pixel count, exponents of the best fit functions remain similar, while the coefficients vary. A comparison of the fitted functions and elastic modulus computational results are provided in Table A.1. An influence of the selected threshold on the calculated apparent contact area is shown in Figure A.3.
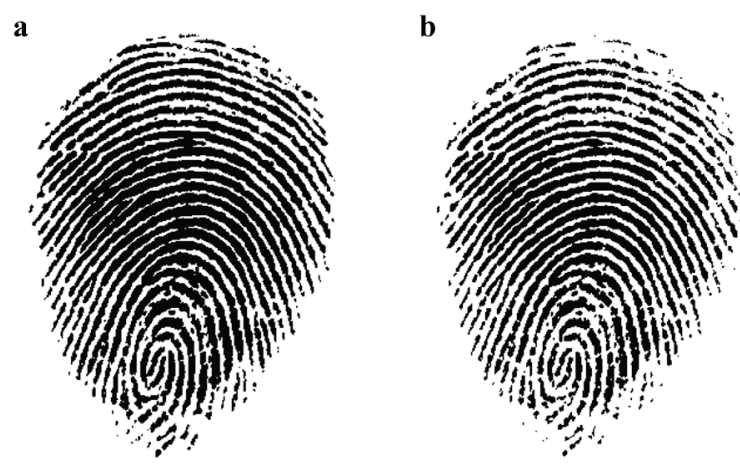

FIGURE A.2 Binary images obtained with the thresholds of 110 (a) and 140 (b) at 5N normal load. 
TABLE A.1 Influence of the threshold selection on calculated apparent contact area and skin Young's modulus.

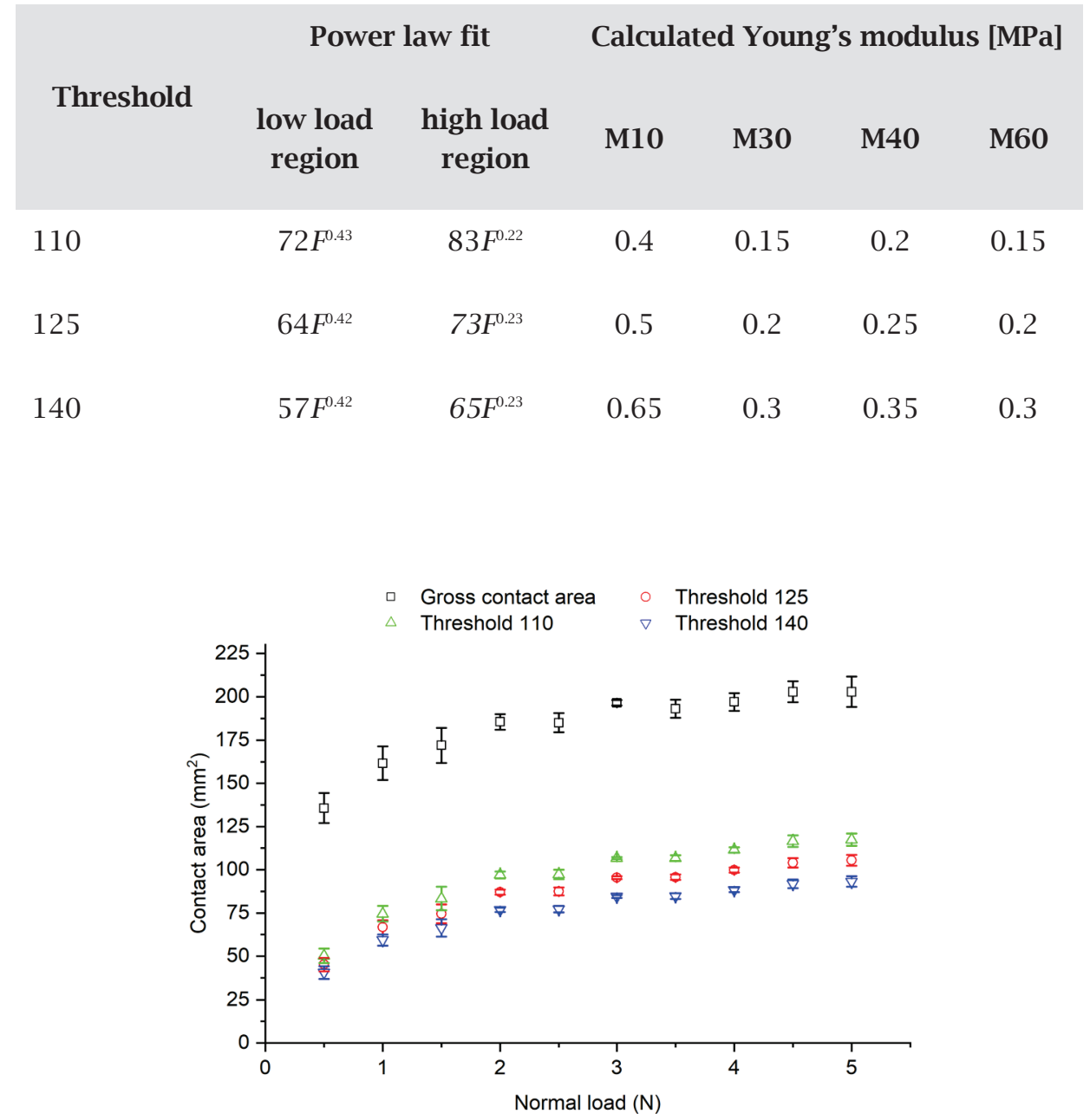

FIGURE A.3 Experimental gross and apparent contact areas calculated with various thresholds. 


\section{PAPER B}

\section{Design of bidirectional frictional behaviour for tactile contact using ellipsoidal asperity micro- textures}

ACCEPTED FOR PUBLICATION IN FRICTION

D. A. Sergachev, D. T. A. Matthews, E. van der Heide 



\section{ABSTRACT}

Tactile perception and friction can be modified by producing a deterministic surface topography. Change of surface feature arrangement and texture symmetry can produce an anisotropic frictional behaviour. It is generally achieved through skin hysteresis by promoting its deformation. This work investigates whether a bidirectional friction can be created with microscale ellipsoidal asperity textures, thus relying on the adhesive component of friction. For this purpose, four textured samples with various asperity dimensions were moulded with a silicone rubber having an elastic modulus comparable to that of the skin. Coefficient of friction measurements were conducted in-vivo in two sliding directions with a range of normal loads up to 4 N. Finite element method was used to study elastic deformation effects, explain the observed friction difference, and predict surface material influence. Measurements performed perpendicular to the asperity major radii showed consistently higher friction coefficients than during parallel sliding. For the larger asperity dimensions, a change of the sliding direction increased friction up to a factor of 2. The numerical analysis showed that this effect is mostly related to elastic asperity deflection. Bidirectional friction differences can be further controlled by asperity dimensions, spacing and material properties.

Keywords:

surface texture; sliding direction; skin friction; elastomer.

\section{INTRODUCTION}

People interact through touch with numerous objects and rely on perceived surface properties in everyday tasks. An individual's judgement of a surface is commonly described by four perceptual scales: hardness, warmth, roughness and stickiness [1,2]. The latter three are directly related to surface topography and, therefore, can be engineered through surface design. However, the relationship between tribological mechanisms and touch perception is not fully understood. There are reported correlations between surface roughness and perceived coarseness [3], as well as between calculated friction coefficients and 
stickiness [4]. Ndengue et al. [5] have shown that a change of material from wood to polyurethane resin affects the way the surfaces are described by volunteers, even though topographical features remained the same. Recently Massimiani et al. [6] and Faucheu et al. [7] performed elaborate studies on the connection between perception and surface texture dimensions. Interestingly, the textures with micro-scale features and spacing below $160 \mu \mathrm{m}$ have shown not only the lowest friction but were uniformly categorized by volunteers as smooth and most likeable.

Friction forces acting on a finger pad can be represented as a sum of interfacial adhesion and deformation terms $[8,9]$. The adhesion term remains predominant during sliding against surfaces of various roughness [10-13]. Hysteresis deformation becomes significant with an addition of macro-scale surface texturing or features with dimensions in the order of millimetres [14,15]. Tomlinson et al. [11] found an interlocking friction mechanism to become predominant during finger pad sliding over triangular ridges with the heights over $42.5 \mu \mathrm{m}$.

With adhesion being predominant and directly proportional to the real contact area, finger pad friction can be modified by controlling surface roughness [10,16,17], or for example by micro-texturing [18-21]. The first approach generally assumes stochastic roughness, which is relatively easy to manufacture, but its frictional performance is difficult to predict and reproduce due to a featureless topography. On the other hand, texturing allows to design the contact conditions, develop contact models and estimate frictional behaviour $[18,22,23]$.

By introducing a surface texture, frictional behaviour can be changed with respect to the sliding direction. It is generally achieved through the creation of wavy or parallel-ridge textures [14,24,25], which utilize skin hysteresis either to reduce the contact area during sliding or to promote deformation. While more complicated textures with anisotropic frictional behaviour can be produced, such as hook-like spines [26] or shark denticle [27], experimental work with a skin has not been performed yet. Moreover, there are no studies on the feasibility of bisymmetric microtextures and their frictional performance, known to the authors.

The aim of the current work is to investigate whether a bidirectional frictional behaviour can be created with a microscale surface texture. This implies that difference in friction is achieved not by the deformation of 
the finger pad skin, but by changing the contact area. For this purpose, textures composed of ellipsoidal asperities were produced on a soft silicone rubber with an elastic modulus comparable to that of the skin. The nature of anisotropic frictional behaviour can be explained though the analysis of friction measurements and the aid of the finite element simulations. Micro-textures with frictional dependency on sliding direction can possibly be used to direct the tactile interaction or improve the grip in specific direction without making significant changes to a product appearance or its feel.

\section{MATERIALS AND METHODS}

\subsection{Materials}

Four texture designs with square-packed ellipsoidal asperities were used for this study. A lateral spacing of $200 \mu \mathrm{m}$ was kept for all the samples, while ellipsoid dimensions varied. All ellipsoidal textures in this study have the same minor asperity radius of $25 \mu \mathrm{m}$ and two major radii of 50 and $75 \mu \mathrm{m}$ (Figure 1). Each of those designs were produced with the heights of 30 and $50 \mu \mathrm{m}$. The samples are referred in the text by their major asperity length as E100 and E150 and by their height as H30 and H50 respectively. The surface topography was obtained using a 3D LED confocal microscope S neox (Sensofar, Spain).
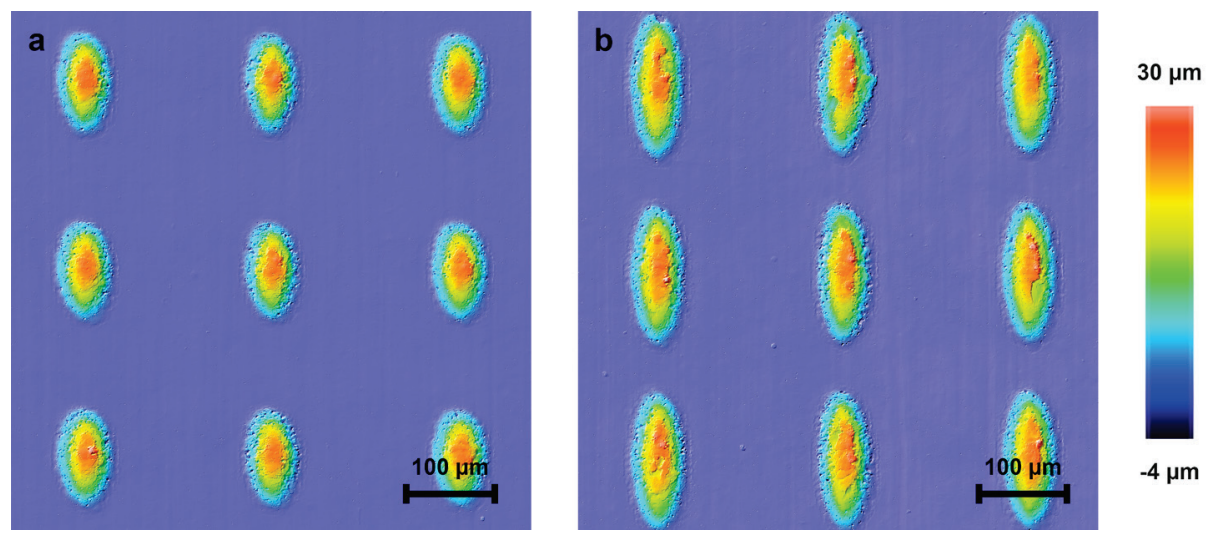

FIGURE 1

Ellipsoidal asperity textures with major radius of 50 (a) and $75 \mu \mathrm{m}$ (b). 
Stainless steel was laser textured to produce negative texture designs, which were used for moulding of silicone rubber master samples. In turn, master samples were replicated by double moulding technique, using polyurethane resin Smooth-Cast ${ }^{\circledR} 305$ (Smooth-on, USA) for a negative intermediate mould. This step was introduced to reduce adhesion during demoulding process and improve microtexture reproducibility. Specimens with a thickness of $10 \mathrm{~mm}$ were produced using commercial silicone rubber Mold Max $^{\mathrm{TM}}$ MM10 (Smooth-on, USA), which has an approximate elastic modulus of $0.2 \mathrm{MPa}$. It was selected to promote deformation of elastomer structure over the skin during sliding.

\subsection{Friction measurements}

Friction measurements were performed in-vivo on a setup designed by Klaassen et al. [28] with a linear reciprocating tribometer depicted on Figure 2. A transducer with an attached sample is mounted on a mechanical hinge driven by a spindle. The right hand is placed palm up on a stage below, with an index finger positioned on a 30-degree wedge. The sample is positioned on top of the finger pad and the normal load is applied with static weights.

The friction behaviour between textures and index finger pad was investigated with one subject, one of the authors of this study. The samples were moved from the subject's body with a constant velocity of $2 \mathrm{~mm} / \mathrm{s}$ and an initial acceleration of $2 \mathrm{~mm} / \mathrm{s}^{2}$. The stroke distance was

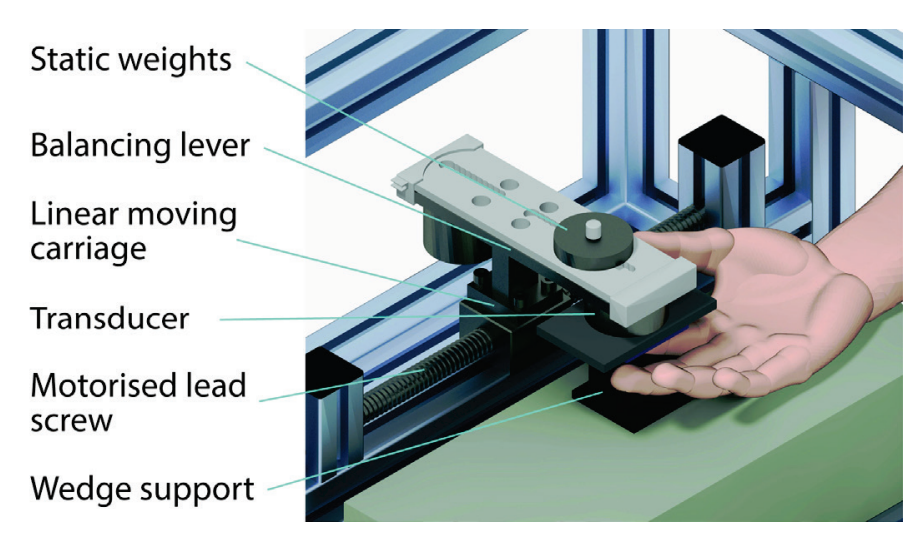

FIGURE 2 Schematic representation of the test setup. 
$20 \mathrm{~mm}$. The hinge with the sample was lifted after each measurement and returned to its home position.

Samples were tested with a range of normal loads from $0.2 \mathrm{~N}$ to $2 \mathrm{~N}$ with a step of $0.2 \mathrm{~N}$ and further up to $4 \mathrm{~N}$ with a step of $0.5 \mathrm{~N}$. Three consecutive measurements were performed with each normal load. Before the start of the measurements 3 preparatory strokes were performed at $0.2 \mathrm{~N}$ load to introduce a sebum layer on a sample surface and ensure consistent conditions across normal loads.

Friction measurements were split into two sets, with texture asperity heights of 30 and $50 \mu \mathrm{m}$ respectively. Each set consisted of two textured surfaces with different asperity length produced in duplicate and a reference non-textured sample. The measurements were performed in two sliding directions: parallel and perpendicular to the major asperity radii. Each sample set was tested in two different days to confirm that the results are related to texture design and independent of skin properties. This constitutes four measurement series which are denoted as S1 to S4 in the study. The sample order was randomized during each series, while the normal load was increased consequently. Randomization of normal loads was not viable for a chosen approach. After removal of the normal load finger pad takes time to recover to its original shape due to its viscoelastic properties. Therefore, it would either introduce random deviations in friction measurements or significantly increase testing times.

The samples were wipe-cleaned with acetone and alcohol, rinsed with demineralized water and kept in the vacuum chamber for 12 hours prior to the measurements to prevent rubber swelling.

Experiments were performed in ambient conditions. Average temperature and relative humidity were calculated as $22 \pm 1{ }^{\circ} \mathrm{C}$ and $43 \pm 7 \%$ respectively. Hands were washed with soap and air dried for 15 minutes before each sample measurement. Skin hydration level was checked between the measurements with Corneometer CM 825 (Courage + Khazaka GmbH, Germany). Corneometer values remained stable between days of measurements with a mean of $50 \pm 4$ arbitrary units. 


\subsection{Data processing}

A 6-axis Mini40 transducer (Schunk, Germany) was used to measure the forces at $100 \mathrm{~Hz}$ sampling rate. The load cell has a resolution of $6 \mathrm{mN}$ and $2 \mathrm{mN}$ in the normal and tangential directions, respectively.

The data from the individual measurement strokes was filtered to find the average dynamic friction values for each normal load. A MATLAB code was used to separate the stable region of the curve during sliding from the initial slope, which corresponds to elastic deformation. A typical example of measured signals is shown in Figure 3. Moreover, a high wavelength noise was induced by the linear driving mechanism, which corresponds to the pitch distance of the shaft and has a constant frequency of $0.5 \mathrm{~Hz}$ with the used sliding velocity. A sine wave fit with a given frequency was determined by a MATLAB code for each measurement to determine a phase offset and an amplitude. The corresponding sine function was subtracted from the original signal to reduce the standard deviation. The calculated average dynamic friction values were not influenced by this operation.

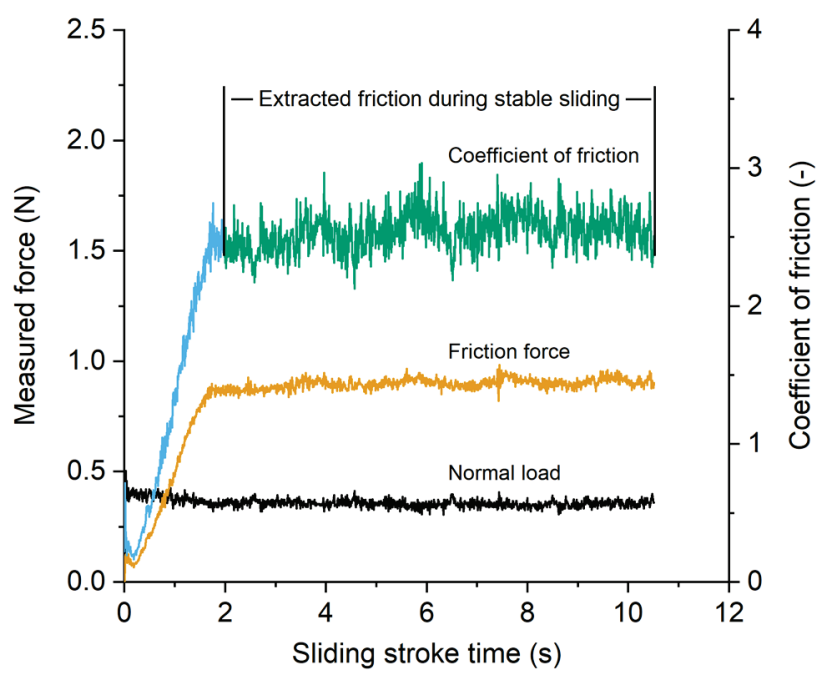

FIGURE 3 An example of raw force signals with calculated and extracted dynamic friction coefficient. Textured sample E150 H50 tested perpendicular at $0.4 \mathrm{~N}$ normal load. 


\subsection{Design of computational experiment}

The objective of the finite element method (FEM) analysis was to study contact behaviour of a single asperity under shear and normal loading. A parametric study was performed to characterize the influence of asperity size, Young's modulus and sliding direction on the contact area development.

The model is represented by two bodies: an asperity and a sliding block, both modelled as isotropic linear elastic elements (Figure 4). An asperity was meshed with tetrahedral-type elements with an average size of $10 \mu \mathrm{m}$ and distortion control was used to prevent excessive mesh warping. The loading block was meshed with hexahedral-type elements of the same size.

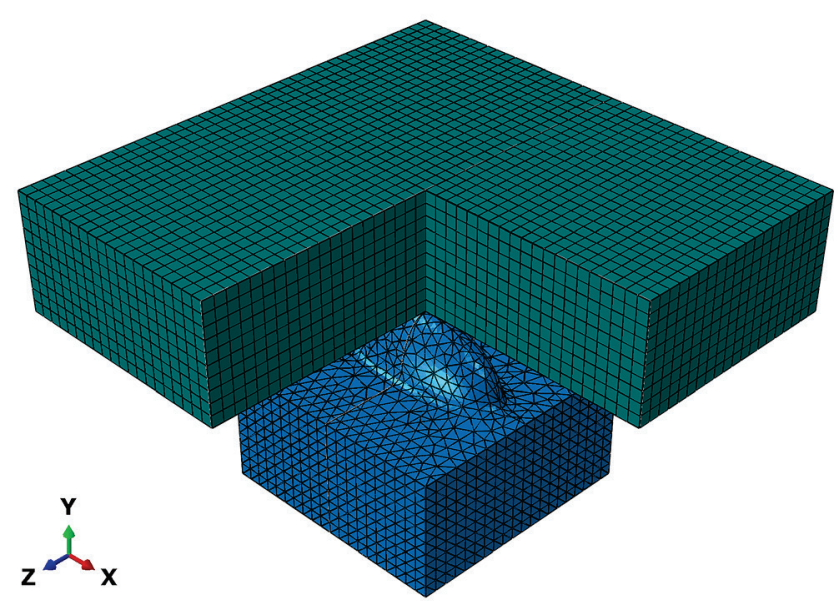

FIGURE 4 An example of an element layout and body meshing for E150 H50. Upper block is shown under a section view.

Interaction between two surfaces was defined by penalty friction formulation. To represent an adhesive friction component, which remains predominant in studied contact conditions, the friction coefficient was set to 1 , while the maximum shear stress was limited to $10 \mathrm{kPa}$. Therefore, interfacial shear force remained mostly independent of contact pressure, simulating adhesive contact. Surface self-contact for the asperity body was set to frictionless to reduce simulation times. 
Initial boundary conditions were specified to restrict body movement. Asperity block was fixed at the bottom plane and its sides were not allowed to move in XZ plane. Each finite element method analysis consisted of two calculation steps with prescribed displacement boundary conditions for the upper body:

- Vertical translation to induce normal load;

- Horizontal translation at constant normal displacement which ensured contact sliding.

There was no intention to simulate the mechanical behaviour of the skin with the current FEM study; yet only to represent similar normal displacement. Therefore, the upper body was assumed as an elastic body with Young's modulus of $0.4 \mathrm{MPa}$, which was estimated in our previous study [29]. Asperity properties were set according to the tested material with the elastic modulus of $0.2 \mathrm{MPa}$. Poisson's ratio of 0.49 was used for both bodies [30,31]. For a continuous sliding inertial forces do not alter solution significantly. Therefore, fixed mass scaling was applied to the entire model to reduce calculation times.

\section{RESULTS AND DISCUSSION}

\subsection{Friction measurements}

Calculated friction coefficients for the textures with asperity height of 30 $\mu \mathrm{m}$ are presented in Figure 5. The reference surfaces, selected to represent the maximum real area of contact, showed friction coefficient values that ranged from about 6.5 at the lower end of the normal force scale down to about 2.5 for the higher end of the scale. All non-textured reference samples follow a power law trend commonly observed for an adhesion-based frictional response. Part of the differences in the resulting coefficient of friction for the reference surfaces might well be due to variation in local interfacial shear stress or day-to-day differences in elastic conditions of the finger pad, yet part was also due to differences in roughness of the reference surfaces. The reference sample tested during the first measurement series showed considerably lower friction coefficient. Its surface roughness average $S_{a}$ was measured around 150 $\mathrm{nm}$, while for other reference samples it was between 40 and $60 \mathrm{~nm}$. 
Reduction of the friction coefficient with increase of roughness is consistent with literature, see e.g. [10,16,17,19].

Both textured surfaces showed the lowest friction during parallel sliding. It increased with normal load up to a maximum of 2.7 and 2.3 for E100 (Figure 5a) and E150 (Figure 5b), respectively. After reaching these peak values at $1 \mathrm{~N}$ load, the coefficient of friction declines steadily almost reaching the values of the reference sample. Such behaviour is explained by the change in a contact state from asperity to full contact. When sliding is performed perpendicular to the major radii, the coefficient of friction increases slightly for all normal loads tested. The greatest difference between sliding directions is observed at normal loads below $0.5 \mathrm{~N}$ and a local peak is less prominent during perpendicular sliding.

Contact with a textured surface can be represented by one of the following three states. At low normal loads skin is fully supported by asperities of the interfacing surface. The real contact area is limited, which results in lower friction forces. With increase of the applied load surfaces deform, and skin reaches a texture valley. Contact transition increases the rate of contact area growth with normal load and leads to the increase in friction. Finally, the contact stabilizes at full contact and further contact area development is restricted. This effect was described previously by other researchers for grooved [23,24] and bump textures [18].

Increase of the asperity heights from 30 to $50 \mu \mathrm{m}$ predictably led to lower friction coefficients during parallel sliding (Figure 6). A one-tailed paired t-test was performed on average values of the measurement series paired by normal load. The p-values for the t-test were lower than 0.05 for both E100 and E150 textures with p-values of 1.2e-5 and 8.6e-6 respectively, which indicate the results are statistically highly significant. The maximum coefficient of friction values of 2.2 and 2.0 were reached during parallel sliding for E100 and E150, respectively. Textures with $50 \mu \mathrm{m}$ asperity heights have a distinctive contact transition stage. The distinction between parallel and perpendicular sliding also became more prominent. The largest difference is observed at lower normal loads, but it diminishes with an increase of applied normal load. 

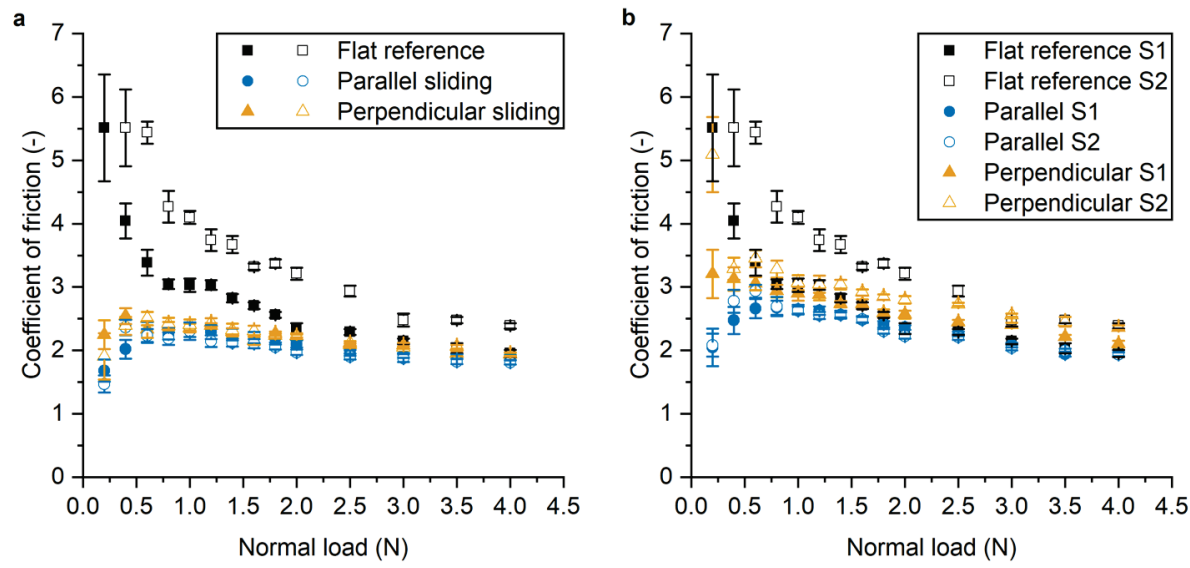

FIGURE 5 Coefficient of friction for $30 \mu \mathrm{m}$ height textures E100 (a) and E150 (b). Corresponding measurement series are denoted as S1 and S2.
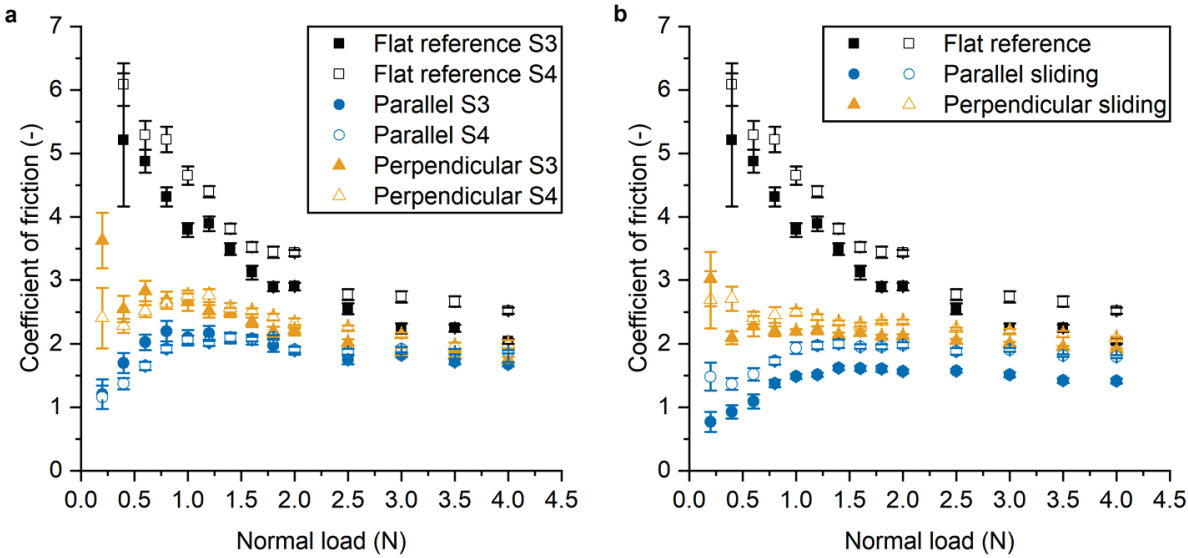

FIGURE 6 Coefficient of friction for $50 \mu \mathrm{m}$ height textures E100 (a) and E150 (b). Corresponding measurement series are denoted as S3 and S4. 
Significant friction difference at lower loads, while surfaces remain in asperity contact, implies that this behaviour is caused by the contact area change and has a predominantly adhesive nature. It is also supported by the fact, that increase of the asperity heights and lateral dimensions is accompanied with reduction of friction, e.g., peak values reduced from 2.7 to 2.2 and from 2.3 to 2.0 for the E100 and E150, respectively. The deformation component of friction positively correlates with deformation, which contradicts the observed behaviour and, therefore, this effect is considered insignificant. Tomlinson et al. [11] reported a considerable contribution to measured friction due to interlocking of the fingerprint ridges during sliding against the triangular-ridged textures with comparable feature dimensions produced on metals. While the interlocking effect can be responsible for the increase of friction with a change of direction, its effect is expected to reach its maximum at full contact with the textures and remain stable through the rest of the normal loads. On the contrary, the directional friction difference reduces upon reaching the full contact state with the textures, suggesting another mechanism responsible for this behaviour.

Contact area with a change of direction can be increased in two possible cases: junction growth and deformation of asperity due to shear. Skin has viscoelastic properties, which implies that during sliding over asperities a junction front can be formed. With the change of sliding direction, the junction contact area increases leading to a higher friction value. In this case, an increase of ellipsoid major radius would lead to an increase of the contact area. Contrary to that, the experimental results show lower or comparable coefficient of friction for the longer asperities.

Alternatively, a contact area rise can be the result of elastic asperity deformation due to shear stresses. Resistance to shear is proportional to the lateral cross section of the asperity. Therefore, an increase of ellipsoid major radius will increase the force required to deform the asperity. K. Brörmann performed friction measurements with cylindrical micro-pillar textures [32]. Each pillar had a diameter and height of 50 and $20 \mu \mathrm{m}$, respectively. They observed elastic deformation of the asperities due to shear and change of contact area accompanied by a stick-slip phenomena. 


\subsection{Modelling results}

The difference between parallel and perpendicular sliding directions was analysed with finite element simulations. A series of calculations was performed for each ellipsoidal asperity size. Normal displacement was changed iteratively from 5 to $40 \mu \mathrm{m}$ with a step of $5 \mu \mathrm{m}$.

For elliptic structures with the height of $30 \mu \mathrm{m}$, change of sliding direction did not affect the results. Both contact area and normal loads remained similar. However, for the asperities of $50 \mu \mathrm{m}$ height the deformation with the change of sliding direction became evident. Deformed geometry of the ellipsoid at the end of each modelling step is shown in Figure 7. Increased asperity height under interfacial shear forces promoted an elastic deflection, which changed the contact state during tangential sliding.

Figure 8 compares development of the normal load and contact area between sliding directions for E150 H30 and E150 H50 for the same normal displacement of $25 \mu \mathrm{m}$. For the parallel sliding case there is a slight increase of normal load at the start of the tangential translation step. However, it returns to the initial value. Its calculated contact area also remains stable. Change of the sliding direction and resulted asperity deformation cause the reduction of the normal load, where it stabilised for the rest of the motion. This change is negligible for smaller asperity but is significant at asperity height of $50 \mu \mathrm{m}$ with a drop from 0.24 to $0.14 \mathrm{mN}$. It also led to fluctuations and a gradual increase of the contact area.

The data from the stable region during tangential sliding was averaged for each successful simulation run to represent the contact area as a function of normal load and is shown in Figure 9. At low normal loads there is no directional difference in contact area. However, as the total shear force increases with the contact area, the asperity starts to bend. It leads to substantially higher contact areas and displacement with respect to the normal load. For longer asperities, the contact area nearly doubles at the same normal load as shown in Figure 9b. 

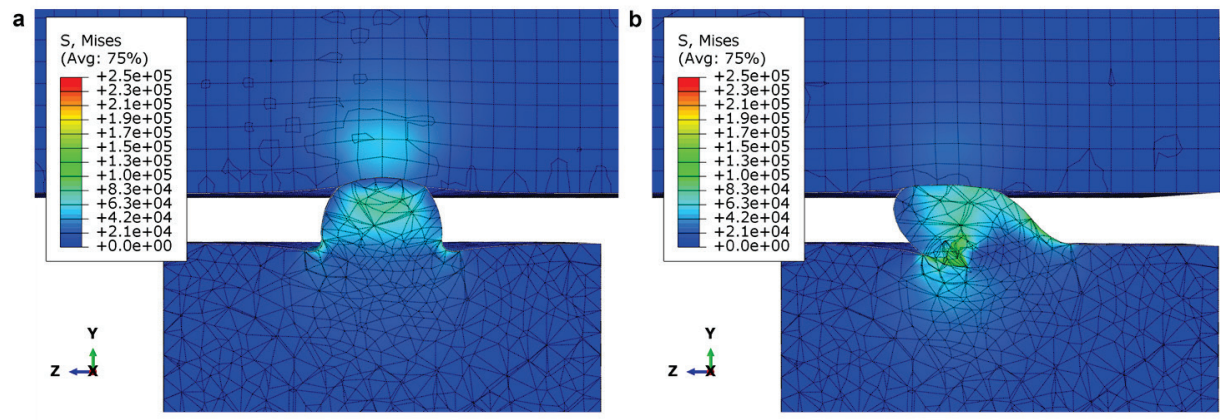

FIGURE 7 Modelled deformation and stresses for E150 H50 at $30 \mu \mathrm{m}$ normal displacement: at the end of the normal (a) and tangential (b) translation steps. Perpendicular sliding, mid-section view.
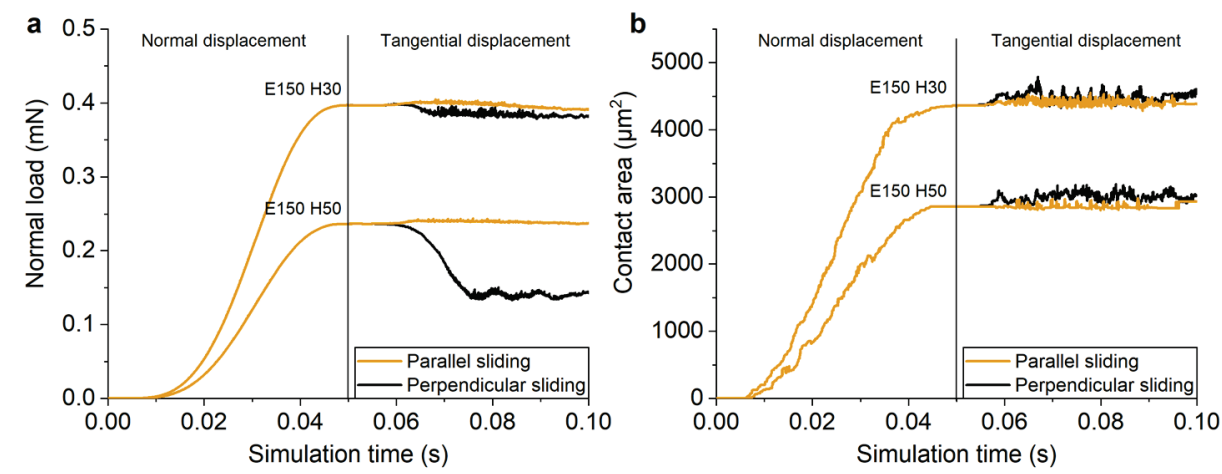

FIGURE 8 Normal load (a) and contact area (b) during numerical simulation for E150 $\mathrm{H} 300$ and $\mathrm{E} 150 \mathrm{H} 50$ at $25 \mu \mathrm{m}$ normal displacement.
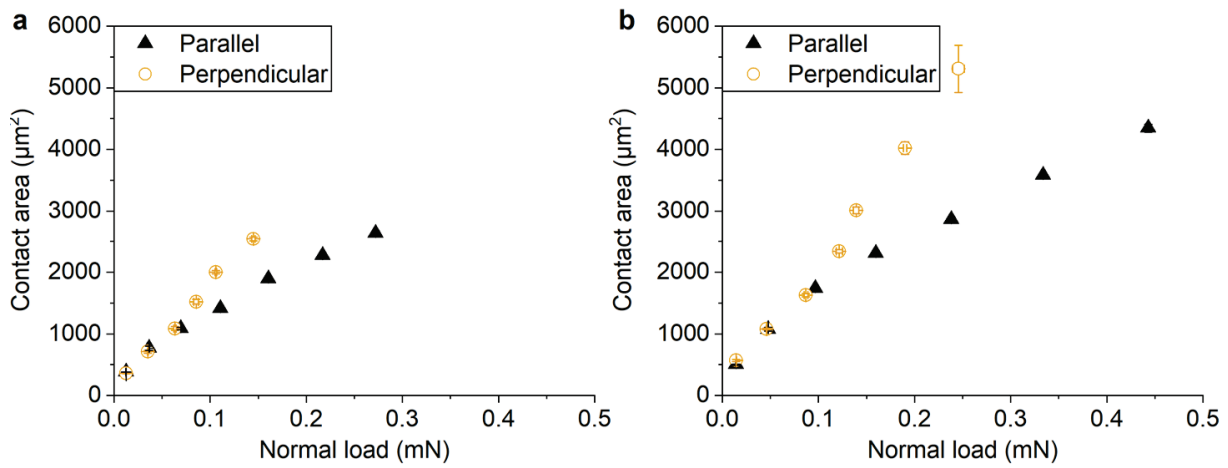

FIGURE 9 Simulated contact area as a function of normal load for E100 (a) and E150 (b). Error bars represent the standard deviation due to fluctuations during sliding. 
Effectively, the asperities in the deformed state can be considered as a different texture with a lower asperity height and larger equivalent radius. Therefore, it results not only in a higher friction at asperity contact state, but the transition to the full contact state also starts at lower normal loads. As expected, an asperity with a larger equivalent radius E150 can support higher normal load before contact transition starts.

A parametric sweep was performed to qualitatively assess the influence of material elastic modulus on the deformation. Figure 10 shows that material Young's modulus significantly affects the shear force required for the deformation to occur. The relative contact area difference between sliding directions is also reduced with increase of elastic modulus, implying that a higher directional effect can be achieved with compliant materials. The maximum contact area with an asperity is limited by the transition to the full contact state, which is directly related to the tangential force. Once the full contact state is reached, a further increase of contact area for asperity becomes negligible and, therefore, the directional difference is less likely to be observed.

Computational results are in a good agreement with experimental measurements. Asperity deflection explains the principal mechanism

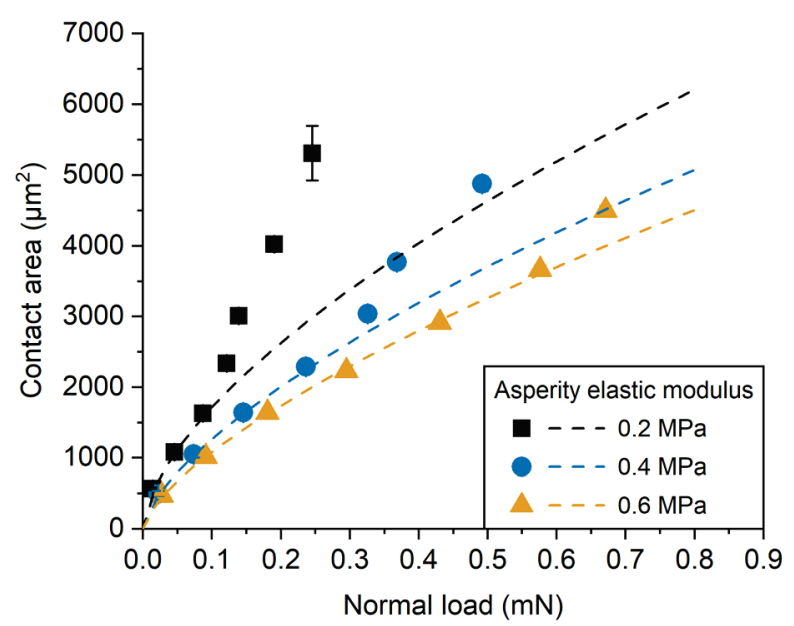

FIGURE 10 Influence of asperity elastic modulus on deformation. Dashed lines show the best fits for parallel sliding, while points represent simulation results for perpendicular sliding direction. 
responsible for the friction gain with the change of the sliding direction. Estimated increase in contact area for E100 and E150 approaches the factors of 1.5 and 2 respectively, which matches the relationship between friction coefficients for these textures. Furthermore, the maximum difference is reached at asperity contact state before the transition to full contact, which correspond to the low normal load region on the friction graphs in Figure 6.

\section{CONCLUSIONS}

- Change of the sliding direction against ellipsoidal asperity textures increased the friction coefficient up to a factor of 2 . The highest difference was measured for the texture with the major radius and height of $75 \mu \mathrm{m}$ and $50 \mu \mathrm{m}$, respectively.

- Observed distinctions in tactile friction between sliding directions have predominantly adhesive nature and became the consequence of the contact area change. During perpendicular sliding, the contact area was increased due to asperity elastic deflection.

- The highest difference between parallel and perpendicular sliding directions is predicted during asperity contact before the transition to full contact state happens. The effect increases with asperity major radius and height.

- FEM simulations show a significant influence of material elastic modulus on the normal load at which the asperity deformation starts. In the absence of asperity deformation, friction differences due to sliding direction are expected to depend on skin properties, such as its tangential stiffness and viscoelastic response.

\section{Ethical approval}

All procedures performed in the current studies involving human participant were in accordance with the ethical standards of the institutional and/or national research committee and with the 1964 Helsinki declaration and its later amendments or comparable ethical standards. The subject provided a written informed consent. None of the experiments were invasive or harmful physiologically or psychologically. 


\section{Acknowledgements}

This work was supported by INTERREG V-A Deutschland - Nederland program MOVERO under the project number 142091.

\section{REFERENCES}

[1] M. Holliins, R. Faldowski, S. Rao, F. Young, Perceptual dimensions of tactile surface texture: A multidimensional scaling analysis, Percept. Psychophys. 54 (1993) 697-705. doi:10.3758/BF03211795.

[2] M. Hollins, S. Bensmaïa, K. Karlof, F. Young, Individual differences in perceptual space for tactile textures: Evidence from multidimensional scaling, Percept. Psychophys. $62 \quad$ (2000) 1534-1544. doi:10.3758/BF03212154.

[3] L. Skedung, K. Danerlöv, U. Olofsson, C. Michael Johannesson, M. Aikala, J. Kettle, M. Arvidsson, B. Berglund, M.W. Rutland, Tactile perception: Finger friction, surface roughness and perceived coarseness, Tribol. Int. 44 (2011) 505-512. doi:10.1016/j.triboint.2010.04.010.

[4] L.E.M. Grierson, H. Carnahan, Manual exploration and the perception of slipperiness, Percept. Psychophys. 68 (2006) 1070-1081. doi:10.3758/BF03193710.

[5] J.D. Ndengue, I. Cesini, J. Faucheu, E. Chatelet, H. Zahouani, D. Delafosse, F. Massi, Tactile Perception and Friction-Induced Vibrations: Discrimination of Similarly Patterned Wood-Like Surfaces, IEEE Trans. Haptics. 10 (2017) 409-417. doi:10.1109/TOH.2016.2643662.

[6] V. Massimiani, B. Weiland, E. Chatelet, P.H. Cornuault, J. Faucheu, F. Massi, The role of mechanical stimuli on hedonistic and topographical discrimination of textures, Tribol. Int. 143 (2020) 106082. doi:10.1016/j.triboint.2019.106082.

[7] J. Faucheu, B. Weiland, M. Juganaru-Mathieu, A. Witt, P.H. Cornuault, Tactile aesthetics: Textures that we like or hate to touch, Acta Psychol. (Amst). 201 (2019) 102950. doi:10.1016/j.actpsy.2019.102950.

[8] S.A. Johnson, D.M. Gorman, M.J. Adams, B.J. Briscoe, The friction and lubrication of human stratum corneum., Tribol. Ser. 25 (1993) 663-672. doi:10.1016/S0167-8922(08)70419-X.

[9] E. Van Der Heide, X. Zeng, M.A. Masen, E.V.A.N.D.E.R. Heide, X. Zeng, M.A. Masen, Skin tribology: Science friction?, Friction. 1 (2013) 130-142. doi:10.1007/s40544-013-0015-1.

[10] S. Derler, L.C. Gerhardt, A. Lenz, E. Bertaux, M. Hadad, Friction of human skin against smooth and rough glass as a function of the contact pressure, Tribol. Int. 42 (2009) 1565-1574. doi:10.1016/j.triboint.2008.11.009. 
[11] S.E. Tomlinson, M.J. Carré, R. Lewis, S.E. Franklin, Human finger contact with small, triangular ridged surfaces, Wear. 271 (2011) 2346-2353. doi:10.1016/j.wear.2010.12.055.

[12] M.A. Darden, C.J. Schwartz, Skin tribology phenomena associated with reading braille print: The influence of cell patterns and skin behavior on coefficient of friction, Wear. 332-333 (2015) 734-741. doi:10.1016/j.wear.2014.12.053.

[13] M.J. Adams, B.J. Briscoe, S.A. Johnson, Friction and lubrication of human skin, Tribol. Lett. 26 (2007) 239-253. doi:10.1007/s11249-007-9206-0.

[14] S.E. Tomlinson, R. Lewis, M.J. Carré, S.E. Franklin, Human finger friction in contacts with ridged surfaces, Wear. 301 (2013) 330-337. doi:10.1016/j.wear.2012.12.039.

[15] M. Janko, M. Wiertlewski, Y. Visell, Contact geometry and mechanics predict friction forces during tactile surface exploration, Sci. Rep. 8 (2018) 1-10. doi:10.1038/s41598-018-23150-7.

[16] M.A. Masen, A systems based experimental approach to tactile friction, J. Mech. Behav. Biomed. Mater. 4 (2011) 1620-1626. doi:10.1016/j.jmbbm.2011.04.007.

[17] S.E. Tomlinson, R. Lewis, M.J. Carré, The effect of normal force and roughness on friction in human finger contact, Wear. 267 (2009) 13111318. doi:10.1016/j.wear.2008.12.084.

[18] J. Van Kuilenburg, M.A. Masen, E. van der Heide, The role of the skin microrelief in the contact behaviour of human skin: Contact between the human finger and regular surface textures, Tribol. Int. 65 (2013) 81-90. doi:10.1016/j.triboint.2012.11.024.

[19] S. Zhang, X. Zeng, D.T.A. Matthews, A. Igartua, E. Rodriguez-Vidal, J.C. Fortes, E. van der Heide, Texture design for light touch perception, Biosurface and Biotribology. 3 (2017) 25-34. doi:10.1016/j.bsbt.2017.02.002.

[20] S. Zhang, X. Zeng, D.T.A. Matthews, A. Igartua, E. Rodriguez-Vidal, J. Contreras Fortes, V. Saenz de Viteri, F. Pagano, B. Wadman, E.D. Wiklund, E. van der Heide, Selection of micro-fabrication techniques on stainless steel sheet for skin friction, Friction. 4 (2016) 89-104. doi:10.1007/s40544-016-0115-9.

[21] S. Zhang, X. Zeng, D.T.A. Matthews, A. Igartua, E. Rodriguez-Vidal, J. Contreras Fortes, E. Van Der Heide, Finger pad friction and tactile perception of laser treated, stamped and cold rolled micro-structured stainless steel sheet surfaces, Friction. 5 (2017) 207-218. doi:10.1007/s40544-017-0147-9.

[22] K. Duvefelt, U. Olofsson, C.M. Johannesson, L. Skedung, Model for contact between finger and sinusoidal plane to evaluate adhesion and deformation 
component of friction, Tribol. Int. 96 (2016) 389-394. doi:10.1016/j.triboint.2014.12.020.

[23] A.C. Rodríguez Urribarrí, E. van der Heide, X. Zeng, M.B. de Rooij, Modelling the static contact between a fingertip and a rigid wavy surface, Tribol. Int. 102 (2016) 114-124. doi:10.1016/j.triboint.2016.05.028.

[24] S. Zhang, A. Rodriguez Urribarri, M. Morales Hurtado, X. Zeng, E. van der Heide, The role of the sliding direction against a grooved channel texture on tool steel: An experimental study on tactile friction, Int. J. Solids Struct. 56 (2015) 53-61. doi:10.1016/j.ijsolstr.2014.12.005.

[25] M.A. Darden, C.J. Schwartz, Investigation of friction mechanisms during the sliding of elastomers against hard parallel-ridge textures, Tribol. Int. 63 (2013) 2-7. doi:10.1016/j.triboint.2012.01.005.

[26] Z. Ji, C. Yan, S. Ma, S. Gorb, X. Jia, B. Yu, X. Wang, F. Zhou, 3D printing of bioinspired topographically oriented surfaces with frictional anisotropy for directional driving, Tribol. Int. 132 (2019) 99-107. doi:10.1016/j.triboint.2018.12.010.

[27] L. Wen, J.C. Weaver, G. V. Lauder, Biomimetic shark skin: design, fabrication and hydrodynamic function, J. Exp. Biol. 217 (2014) 16561666. doi:10.1242/jeb.097097.

[28] M. Klaassen, D.J. Schipper, M.A. Masen, Influence of the relative humidity and the temperature on the in-vivo friction behaviour of human skin, Biotribology. 6 (2016) 21-28. doi:10.1016/j.biotri.2016.03.003.

[29] D.A. Sergachev, D.T.A. Matthews, E. van der Heide, An Empirical Approach for the Determination of Skin Elasticity: Finger pad Friction against $\begin{array}{lllll}\text { Textured } & \text { Surfaces, } & \text { Biotribology. } & 18 & \text { (2019) }\end{array}$ doi:10.1016/j.biotri.2019.100097.

[30] R. Fagiani, M. Barbieri, A contact mechanics interpretation of the duplex theory of tactile texture perception, Tribol. Int. 101 (2016) 49-58. doi:10.1016/j.triboint.2016.03.031.

[31] J. Ilg, S.J. Rupitsch, A. Sutor, R. Lerch, Determination of dynamic material properties of silicone rubber using one-point measurements and finite element simulations, IEEE Trans. Instrum. Meas. 61 (2012) 3031-3038. doi:10.1109/TIM.2012.2203449.

[32] K. Brörmann, I. Barel, M. Urbakh, R. Bennewitz, Friction on a Microstructured Elastomer Surface, Tribol. Lett. 50 (2013) 3-15. doi:10.1007/s11249-012-0044-3. 


\section{PAPER C}

\section{Normalising tactile friction}

\section{PUBLISHED AS:}

TRIBOLOGY INTERNATIONAL, VOLUME 161, 2021, ARTICLE 107052

D. A. Sergachev, D. T. A. Matthews, E. van der Heide 



\section{NOMENCLATURE}

symbol description units

\begin{tabular}{|c|c|c|}
\hline$A_{f r}$ & contact area of fingerprint ridges & $\mathrm{m}^{2}$ \\
\hline$A_{g}$ & gross contact area of a finger pad & $\mathrm{m}^{2}$ \\
\hline$A_{r}$ & real contact area & $\mathrm{m}^{2}$ \\
\hline$A_{r_{r e f}}$ & $\begin{array}{l}\text { real contact area of a finger pad with a reference } \\
\text { sample }\end{array}$ & $\mathrm{m}^{2}$ \\
\hline$F$ & applied normal load & $\mathrm{N}$ \\
\hline$F_{a d h}$ & interfacial adhesion friction force & $\mathrm{N}$ \\
\hline$F_{f_{\text {ref }}}$ & friction force, measured against reference sample & $\mathrm{N}$ \\
\hline$F_{f_{\text {tex }}}$ & friction force, measured against a textured sample & $\mathrm{N}$ \\
\hline$k$ & power-law coefficient for contact area & $\mathrm{m}^{2} \mathrm{~N}^{-m}$ \\
\hline$m$ & power-law load index for contact area & - \\
\hline$p_{r}$ & mean real contact pressure & $\mathrm{Pa}$ \\
\hline$p_{f r}$ & mean ridge contact pressure & $\mathrm{Pa}$ \\
\hline$\hat{p}_{f r}$ & $\begin{array}{l}\text { normalised mean ridge contact pressure } \\
\text { normalised by the point of reference }\end{array}$ & - \\
\hline
\end{tabular}


$r \quad$ contact area ratio, calculated by normalisation

$r_{s} \quad$ contact area ratio, calculated with numerical model

$\alpha \quad$ pressure coefficient

$\mu_{\text {ref }}$ friction coefficient, calculated for a reference sample

$\mu_{\text {tex }}$ friction coefficient, calculated for a textured sample

$\tau \quad$ interfacial shear strength

$\mathrm{Pa}$

$\tau_{0} \quad$ intrinsic interfacial shear strength $\quad \mathrm{Pa}$ 


\section{ABSTRACT}

Finger pad friction varies significantly between individuals due to personal differences and environmental effects. For this reason, comparison of the absolute friction values becomes non-informative and is rarely reported in literature. This work investigates whether friction data can be normalised to correlate between individuals. A set of textured samples were tested by 10 volunteers in dry sliding conditions with a range of normal loads up to $4 \mathrm{~N}$. A reference smooth sample was used for each participant to normalise the dataset. The proposed approach allows to eliminate one of the unknown parameters, e.g., finger pad ridge contact area function, and discuss the data with respect to other variables.

Keywords: skin tribology, tactile friction, texture

\section{INTRODUCTION}

The physical properties of a product surface can be perceived by humans through active touch and tactile exploration of the surface by the finger pad. An important requirement for texture discrimination is a relative sliding movement between the finger pad and a surface [1]. Resulting friction and normal forces serve as a stimulus, which activate groups of mechanoreceptors located just below the skin surface. Surface textures can be designed to modify this frictional behaviour with the aim to enhance the product tactile perception or improve its handling performance by making it more sticky or slippery. For research and development purposes texture design might be validated by friction experiments. The experimental data could, however, easily result to a very broad set of friction values when compared between individuals [2-4]. The variance is partially caused by change in the operational conditions and partially by differences in the finger pad structure from person to person. Therefore, it is not possible to compare finger pad friction data quantitatively between studies, nor to validate texture designs in a generic way based on friction experiments involving multiple persons. Normalisation of the finger pad friction could allow to compare those results and evaluate the contact conditions at the scale of the finger pad - texture interaction. 
Contact with a finger pad depends on phenomena occurring at multiple length scales. On the macro scale the finger pad deforms producing a generally elliptical shaped gross contact $A_{g}$. As suggested by conventional Hertzian model [5], gross contact area is expected to increase with the normal load to the power of $2 / 3$. However, experimental results show that gross contact area follows a power function of the normal load $A_{g}=k \cdot F^{m}$ with the reported exponents $m$ ranging from 0.1 to 0.5 [6-9], suggesting the influence of other parameters. Naturally, people have a different size of the fingertip and, therefore, the finger pad radius differs from person to person. Moreover, the inclination of the finger relative to the surface influences the resulting fingertip radius. Dzidek et al. [8] measured the finger pad gross contact areas at angles of 30 and 45 degrees to the surface. Best power function fits maintained similar exponents $m \approx 0.36$, while the reduction in contact area was associated with a decrease in coefficient $k$. Another influencing parameter, the effective elastic modulus of the skin, is related to finger deformation. At low normal loads, the gross contact area is restricted by harder outer skin layers, while at higher loads, finger pad deformation is restrained by the distal phalanx bone $[6,8]$.

At the meso scale, the finger pad - surface interaction involves deformation of the fingerprint ridges, which form a characteristically shaped contact region. Hereinafter it is also referred to as a ridge contact area. A fingerprint structure has an approximate spacing of 350 to 600 $\mu \mathrm{m}$ between the ridges with respective heights of 40 to $100 \mu \mathrm{m}$ [7,10-12]. Ridge contact area is often assumed to represent the real contact area in contact with smooth flat surfaces $[9,13,14]$. It remains a fraction of the gross contact area with reported values between 0.1 and 0.7 depending on the normal load $[3,9,12,13]$. Ridge contact area follows a similar power function as gross contact i. e. , $A_{f r}=k \cdot F^{m}$, but with a different coefficient and exponent. The exponents reported in literature are generally higher that those calculated for the gross contact and vary from 0.2 to 0.7 $[6,8,9,15]$. A few contact models were proposed for the prediction of the finger ridge contact $[8,12]$. However, they require skin elasticity and fingerprint topography data for the estimation.

The micro-scale is considered in this work for contact with surface features, that have smaller dimensions than the fingerprint ridge at least by one order of magnitude. In this case the ridge is supported along its 
width by multiple asperities and the sum of their contacts defines the real contact area. Without reliable methods to measure the real contact area, only comparative analysis can be performed, e.g. based on friction measurements with designed and deterministic textures. Given the predominantly adhesive nature of the finger pad friction [16-18], introduction of surface texture allows to reduce and limit the real contact area, which allows to study the contact mechanics on the microscale $[7,12,17]$. Nevertheless, to predict friction for an individual, one requires to know the contact area at meso scale, interfacial shear strength and effective elastic modulus of the skin at microscale.

Coefficient of friction is rarely compared between volunteers due to its variation based on personal and environmental differences [19,20]. Veijgen et al. [21] reported a linear relationship between corneometer hydration values and dynamic friction coefficient. M. Arvidsson et al. proposed to normalise the friction measurements with the moisture level from the corneometer [22]. They observed the linear increase of the friction coefficient with the rise in skin hydration level for the same sample and finger. Moreover, the data between several participants was compared after linear normalisation of the friction coefficients, based on the ratio between mean total and mean individual values.

The aim of the current work is to investigate whether tactile friction against textured surfaces can be compared between individuals after data normalisation with respect to discussed variables. The method that is used in the current work is original for skin friction and based on experimental work that uses deterministic surface textures and a smooth reference sample. Reference sample is used to remove the macro- and meso-scale friction components from the observed friction measurements and, therefore, compare micro-scale behaviour between individuals. The method is not aimed to standardise finger pad friction measurements but is proposed to increase the relevance of friction coefficient in comparative studies. 


\section{MATERIALS AND METHODS}

\subsection{Materials}

Surface texture designs were selected based on our previous studies $[23,24]$ with the aim to represent three distinctive contact states, i.e., contact with asperity features, full contact with a texture valley and a gradual transition between the two. This approach allows to estimate microscale deformation based on friction measurements. Therefore, three respective textured surfaces and a non-textured reference were used in this study. All textures consist of square packed evenly distributed asperities (Figure 1). Textures S100 and S200 have spherical asperities with a lateral spacing of 100 and $200 \mu \mathrm{m}$ respectively. Bumps have the same tip radius of $27 \mu \mathrm{m}$ and height of $22 \mu \mathrm{m}$. Sample E200 consists of ellipsoidal asperities placed with a spacing of $200 \mu \mathrm{m}$ between each other. Ellipsoids have a minor and major lengths of 50 and $100 \mu \mathrm{m}$ respectively. The ellipsoidal texture also had the highest feature height of $47 \mu \mathrm{m}$. Flat reference samples had an average roughness of $70 \mathrm{~nm}$. The surface topography was obtained with a 3D LED confocal microscope $S$ neox (Sensofar, Spain).

Textures were replicated on a thin silicone rubber to promote a compliant adhesive contact and prohibit a rise of the deformation friction. Samples were produced by taking a $2 \mathrm{~mm}$ thick imprint from a textured embossing roll with commercial silicone rubber ALPA-SIL 97071-3 (CHT Group, Germany). It has a Shore-A hardness of 45 and an estimated Young's modulus of $1.5 \mathrm{MPa}$. Silicone was cut into equal pieces of $50 \times 50 \mathrm{~mm}$ and glued to polycarbonate mounting plates using a silicone rubber adhesive Sil-Poxy ${ }^{\mathrm{Tm}}$ (Smooth-on, USA). Texture features were aligned to perform sliding parallel to the vertical direction of Figure 1. Therefore, elliptical texture was moved along the major asperity radius to reduce deformation friction component.

Samples were wet wiped with tissues soaked in acetone and alcohol and rinsed with demineralised water. Prior to the friction measurements the samples were put in a vacuum chamber for at least 12 hours to prevent possible rubber swelling. 

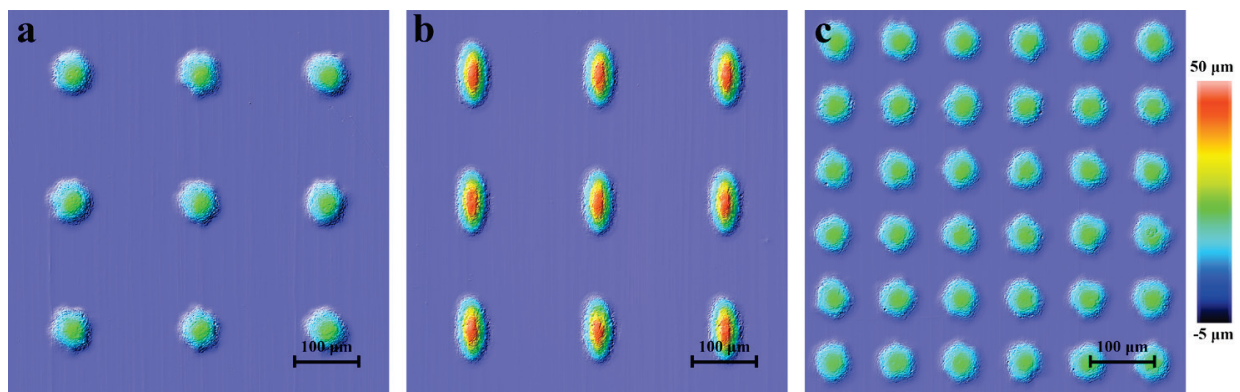

FIGURE 1

Surface topography of S200 (a), E200 (b) and S100 (c) obtained with confocal microscope.

\subsection{Friction measurements}

Friction measurements were performed with a setup designed by Klaassen et al. [19]. The equipment was customised for finger pad sliding tests. The instrument consists of a chamber with a lead screw driven linear stage, which carries a balancing lever with a loadcell on one end and a counterweight on the other (Figure 2). The hand is placed on a stage palm up with an index finger positioned on a 30-degree wedge. The sample plate attached to the transducer is lowered on top of a finger pad while the normal load is set with a static weight. An additional motor was mounted on a stage to set the sensitivity drift correction, control the lever lowering speed and lock its position angle during carriage return.

Measurements were performed in-vivo with a test group of 10 adult and healthy volunteers (8 males and 2 females), represented within the ages

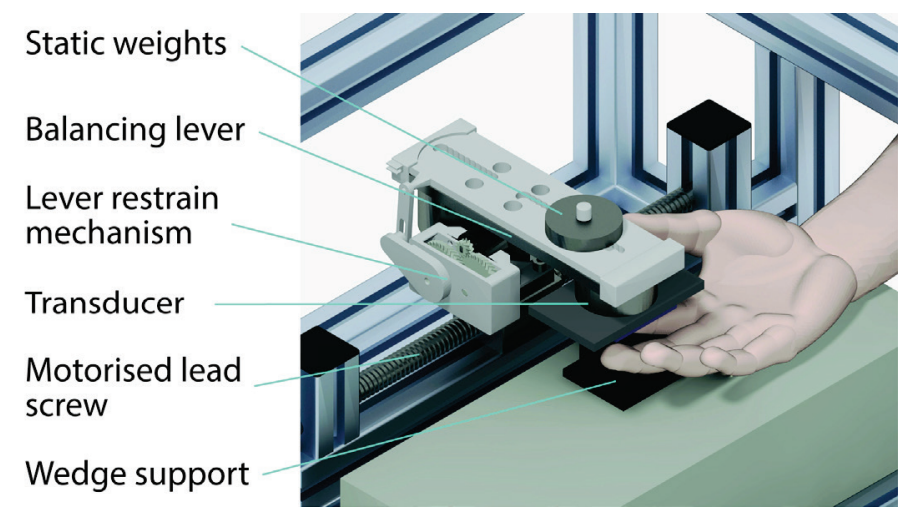

FIGURE 2 Schematic representation of the test setup. 
range of 25 to 55 . There were no specific selection criteria due to the fact, that finger pad radius, topography and skin properties vary between individuals, which inevitably results in different contact areas, contact pressures, and frictional behaviour. \{The tested hypothesis is not related to the properties of human skin but follows from the adhesive nature of the contact.\} Before the experiment, the test procedure was thoroughly explained to each participant and an informed consent was taken. Each participant had his own set of four samples. Sample order was randomised and tested within the same day per person.

The tests were carried out in an ambient environment with the temperature and humidity being measured. Prior to performing measurements with each sample, a participant was asked to wash hands with a soap and air dry them for 15 minutes. Afterwards an index finger pad skin hydration level was taken with a Corneometer CM 825 (Courage + Khazaka GmbH, Germany). At the first load of $0.2 \mathrm{~N}$ three preliminary strokes were performed to form a sweat and sebum layer on the surface.

The sample was moved from a subject's body with a constant velocity of $2 \mathrm{~mm} / \mathrm{s}$ and a total sliding distance of $20 \mathrm{~mm}$. Initial acceleration was set to $2 \mathrm{~mm} / \mathrm{s}^{2}$ to ensure that it does not affect the dynamic friction. After each stroke, the sample was lifted, and the stage was returned to its starting position.

The normal load was increased during the measurement series, starting from $0.2 \mathrm{~N}$ and followed by the load range from $0.5 \mathrm{~N}$ to $4 \mathrm{~N}$ with a step of $0.5 \mathrm{~N}$. This decision was made to reduce the variation of the contact area due to the visco-elastic behaviour of the finger pad and conserve the time needed for full finger shape restoration. Three consecutive strokes were performed for each normal load.

\subsection{Data processing}

The forces were measured with a 6-axis Mini40 transducer (Schunk, Germany) at a sampling rate of $100 \mathrm{~Hz}$. The load cell has a resolution of $6 \mathrm{mN}$ and $2 \mathrm{mN}$ in the normal and tangential directions respectively.

A MATLAB script was used to extract the forces and friction coefficient during sliding. That was performed to ensure that initial rising slope, which corresponds to the finger pad deformation, does not influence the 
results. A high wavelength noise of $0.5 \mathrm{~Hz}$ introduced by the guide bearing was removed from the signal. It is recognisable due to the pitch distance of the lead screw of $4 \mathrm{~mm}$ and a number of shaft revolutions per second with a chosen speed. Notably, removing of the specific wavelength does not influence the mean value, but reduces signal deviations. The data from the consecutive measurements at each normal load were used to find arithmetic mean and standard deviation.

\section{CALCULATION}

A minimum dataset that can be used for normalisation consists of friction measurements performed for a single person at a range of normal loads. At least two tested samples are required: a textured sample of interest and a smooth surface to serve as a reference. The obtained data is represented by two friction force functions of the normal load $F_{f_{\text {ref }}}(F)$ and $F_{f_{t e x}}(F)$ for the reference and the textured samples respectively.

The following assumptions are taken for further analysis:

1. Fingerprint ridge contact area $A_{f r}$ as a function of normal load does not change for a person during the whole measurement series.

2. The real contact area with the reference sample $A$ $r_{r e f}$ approaches the ridge contact area of the finger pad $A_{r_{r e f}} \rightarrow$ $A_{f r}$ at all normal loads. It implies that the roughness of the skin on the fingerprint ridges is considered negligible.

3. The deformation component of friction is small and, therefore, the friction force is based purely on adhesion, so that $F_{f}=$ $F_{a d h}=\tau A_{r}$, where $\tau$ is the interfacial shear strength and $A_{r}$ is the real contact area.

With the given assumptions, the measured friction force with a flat sample can be used as a control reference. It can be approximated as a power function of the normal load as $F_{f_{r e f}}(F) \approx k F^{m}$, where $k$ and $m$ are the power-law coefficient and exponent, respectively. Power law fit approximation is performed to reduce calculation errors caused by the deviation of the normal loads during measurements. 
The friction data for the surface of interest can be represented as a unitless real contact area fraction. This designation is chosen instead of a neutral "normalised coefficient of friction" to emphasize the fundamental meaning behind the obtained values. Considering that the interfacial shear strength $\tau$ remains constant for the dataset, the contact area ratio $r$ can be found as a quotient of the friction coefficients or friction forces:

$$
r(F)=\frac{\mu_{t e x}(F)}{\mu_{r e f}(F)}=\frac{\tau A_{r}(F)}{\tau A_{f r}(F)}=\frac{F_{f_{t e x}}(F)}{F_{f_{r e f}}(F)},
$$

where the $F_{f_{t e x}}(F)$ is the measured friction force for the textured sample and $F_{f_{r e f}}(F)$ is a power fit function for the flat reference. Plotting the contact ratio against the normal load allows to evaluate contact area development and compare it between datasets.

However, with a variation of ridge contact area between individuals, the same normal load corresponds to a different ridge pressure. To compare the contact area development at the micro scale, the data can be plotted as a function of the mean ridge pressure. It cannot be found numerically without additional measurements, but it can be substituted by a ratio of the normal and tangential forces. Given that the mean ridge pressure is a function of the normal load as $p_{f r}(F)=F / A_{f r}(F)$, friction data from a reference surface can be used to obtain a dimensionless force ratio:

$$
p_{f r}(F) / \tau=F / \tau A_{f r}(F)=F / F_{f_{\text {ref }}}(F)
$$

Plotting contact area ratio as a function of the force ratio $r\left(p_{f r} / \tau\right)$ removes a power component and allows to compare the micro-contact development between different persons. For the linear elastic case, deformation is linearly proportional to the applied stress. It implies that the for the same textured surface the function of $r\left(p_{f r} / \tau\right)$ is expected to vary between individuals linearly. The difference will be defined by the interfacial shear strength $\tau$ and effective Young's modulus, both can be considered unique and constant for each dataset.

Influence of the effective elastic modulus is generally removed by normalisation of the mean ridge contact pressure by effective elastic 
modulus or maximum pressure $[12,25,26]$. With those values unknown, the axis can be normalised by a $p_{f r} / \tau$ value, which corresponds to a specific contact area ratio. Use of the deterministic surfaces allows to define the value as a force ratio, at which real contact area fraction for two textures is equal. This step allows to compare experimental data with a contact model and discuss the initial assumptions.

\section{RESULTS}

\subsection{Environmental and corneometer measurements}

Measurements with volunteers were performed at different days and times of the day. Room temperature was kept at $22 \pm 0.5{ }^{\circ} \mathrm{C}$ through the measurements. Average relative humidity of $58 \pm 5 \%$ was calculated across participants. Corneometer measurements however, varied significantly between individuals (Figure 3). Most of the volunteers had skin hydration levels with the values between 40 and 60 arbitrary units (AU), which relates to a normal skin condition [27], with subjects V1 and V3 showing extreme values around 30 and 80 AU. For most of the participants skin hydration remained relatively stable during four friction measurement series with a standard deviation below $10 \mathrm{AU}$. Higher variance during the series can be noticed for V4 and V9, however no specific trend was observed.

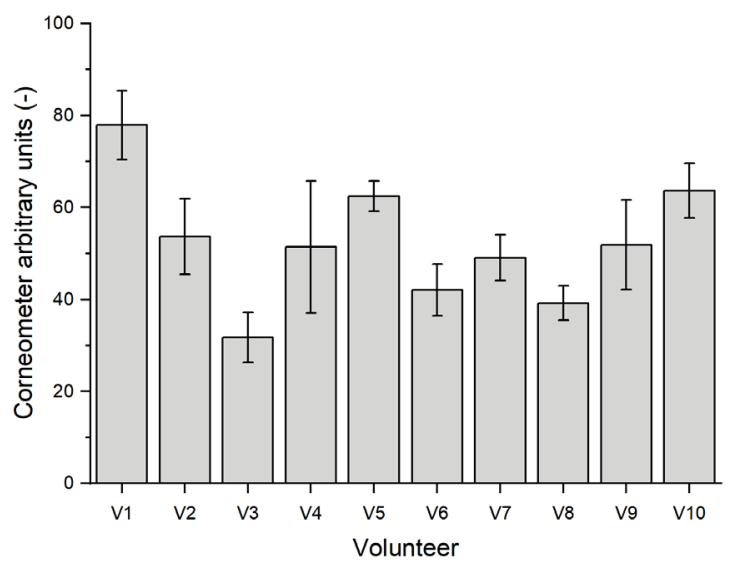

FIGURE 3 Mean corneometer measurements over the test duration with standard deviation for each participant. 


\subsection{Friction measurements}

As anticipated, the experiments showed a large range for the calculated friction coefficients between volunteers. The difference between the highest and the lowest values for the equivalent samples and normal loads reaches a factor of 4 . Flat reference samples showed reduction of friction with increase of the applied normal load, reaching the range of 0.9 - 2.5 at 4N (Figure 4a). Textured samples S200 and E200 exhibited significantly lower coefficient of friction at low normal loads as observed on Figure $4 \mathrm{~b}$ and Figure 4c. However, friction increased with the applied normal loads and stabilised in value ranges of $1.1-2.0$ and $0.7-1.3$ for S200 and E200 respectively. The lowest friction coefficient was observed for the highest density texture S100 and remained independent of normal load at respective values of 0.35 - 1.0 (Figure $4 \mathrm{~d}$ ).

Volunteers can be arranged in descending order by the coefficient of friction for the specific texture and the normal load. However, only the order for the flat reference sample remains consistent for every normal load. The comparison between $0.5 \mathrm{~N}$ and $3 \mathrm{~N}$ normal loads is presented on Figure 4. The sorting order for the textured samples does not follow the same trend. High friction with a reference surface for the specific volunteer does not imply that the same holds true for the textured surfaces. 

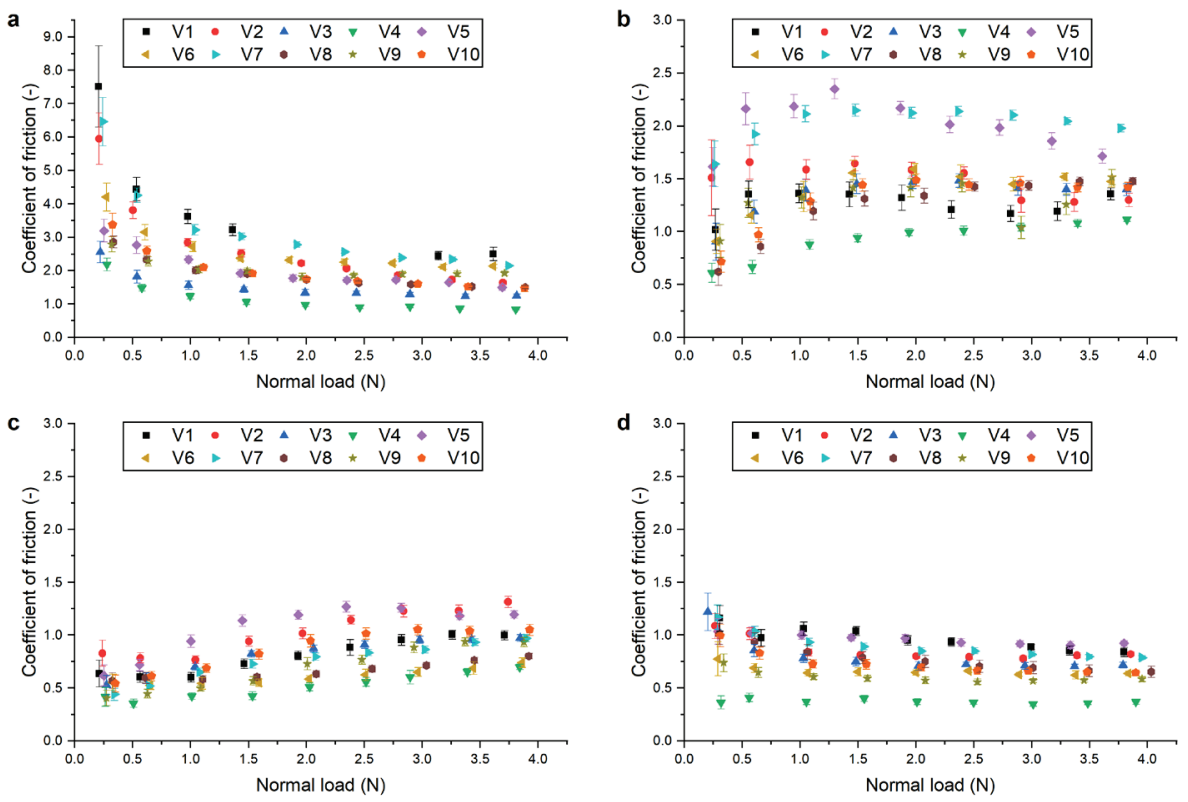

FIGURE 4 Calculated coefficient of friction for all the subjects grouped by surface texture: reference(a), S200 (b), E200 (c), S100 (d). Note the different Y-scale for graph (a).
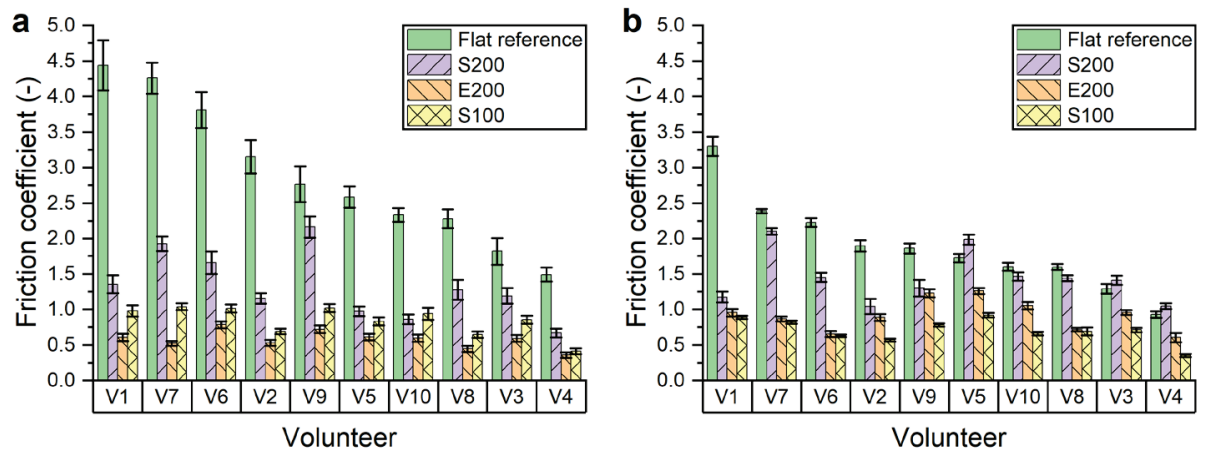

FIGURE 5 Coefficient of friction for each volunteer calculated at $0.5 \mathrm{~N}$ (a) and $3 \mathrm{~N}(\mathrm{~b})$ normal load. The data is sorted in descending order based on the measurements against the flat reference sample. 


\subsection{Power law fit}

The best power law function fit was determined for each volunteer based on the reference friction measurements from Figure $4 \mathrm{a}$. The power law equation for the coefficient of friction $\mu$ was represented as a function of normal load $F$ as: $\mu(F)=k \cdot F^{m-1}$, where $k$ and $m$ are the power-law coefficient and the load index respectively [16]. Consequently, friction force can be represented as $F_{f_{r e f}}(F)=k \cdot F^{m}$. The only three data points which were considered as outliers belong to $\mathrm{V} 1$ at $2.0 \mathrm{~N}, 2.5 \mathrm{~N}$ and $3.0 \mathrm{~N}$ and stand out from the rest of the curve (Figure 4a), therefore they were excluded for the best fit calculation. The comparison of the found functions are provided below in the Table 1. Coefficient of determination $R^{2}$ was used to evaluate the success of the power fits and was above 0.8 for most of the curves. The exponents for the friction forces fits lay in the range from 0.58 to 0.89 , while the latter was obtained from the poor fit for V9. Interestingly, the highest values for the exponent correspond to the subjects with the lowest skin hydration: V3, V6, V8.

TABLE 1 Power fit functions for the friction force measured against the reference sample and coefficient of determination. Failed power fit for V9 marked in italic.

\begin{tabular}{lccccc} 
& $\mathbf{V 1}$ & $\mathbf{V} 2$ & $\mathbf{V 3}$ & $\mathbf{V 4}$ & $\mathbf{V 5}$ \\
$\begin{array}{l}\text { Friction force fit } \\
\text { function, } F_{f}\end{array}$ & $3.64 F^{0.66}$ & $2.89 F^{0.58}$ & $1.60 F^{0.79}$ & $1.27 F^{0.68}$ & $2.23 F^{0.72}$ \\
$\begin{array}{l}\text { Coefficient of } \\
\text { determination, } R^{2}\end{array}$ & 0.96 & 0.99 & 0.91 & 0.94 & 0.94 \\
\hline $\begin{array}{l}\text { Friction force fit } \\
\text { function, } F_{f}\end{array}$ & $2.70 F^{0.79}$ & $3.45 F^{0.65}$ & $2.08 F^{0.75}$ & $2.12 F^{0.89}$ & $2.21 F^{0.69}$ \\
$\begin{array}{l}\text { Coefficient of } \\
\text { determination, } R^{2}\end{array}$ & 0.85 & 0.98 & 0.98 & 0.61 & 0.98
\end{tabular}




\subsection{Normalisation of friction forces}

Obtained power fits are used to normalise the friction data using Eq. 2. The resulted graphs are shown in Figure 6. With this representation the influence of the interfacial shear strength is eliminated, and the contact area development can now be compared between textures and volunteers. The highest contact area ratios are shared by subjects V3, V4 and V5, who have previously shown various hydration levels and friction coefficients. For V3, who had the lowest corneometer readings, it indicates a smaller ridge contact area.

\subsection{Conversion to ridge pressure}

Another power function component was removed from the data by representing contact area fraction as a function of force ratio $r\left(p_{f r} / \tau\right)$ according to the Eq. 3. After plotting the graphs, the change of the trend for the texture E200 shown on Figure $7 \mathrm{~b}$ became evident for all volunteers. It consists of two parts: initial slope comparable to results of S100 on Figure 7c and subsequent change of inclination similar to S200 Figure 7a. On the contrary, surface $\mathrm{S} 100$ has a linear trend for all participants. Normalised data for texture S200 show the largest variation. Volunteers V3, V4 and V5 have a calculated real contact ratio above 1 (Figure 7a), which contradicts the second initial assumption. Interestingly, for two of these participants, V3 and V5, the contact area growth rate decreases around the value of 1.2 . 

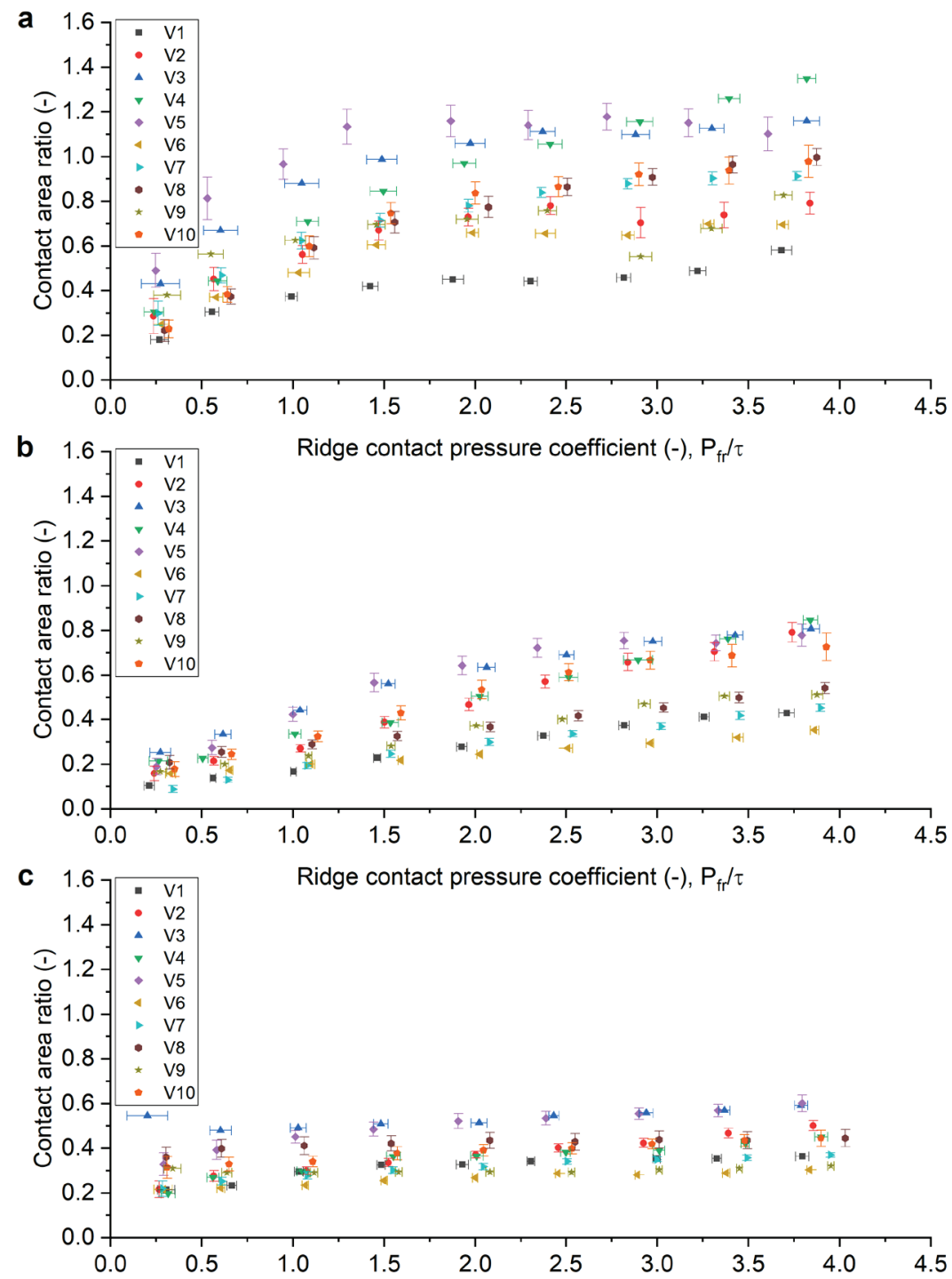

Ridge contact pressure coefficient (-), $P_{\mathrm{fr}} / \tau$

FIGURE 6 Calculated contact area ratio as a function of normal load grouped by texture: S200 (a), E200 (b), S100 (c). 

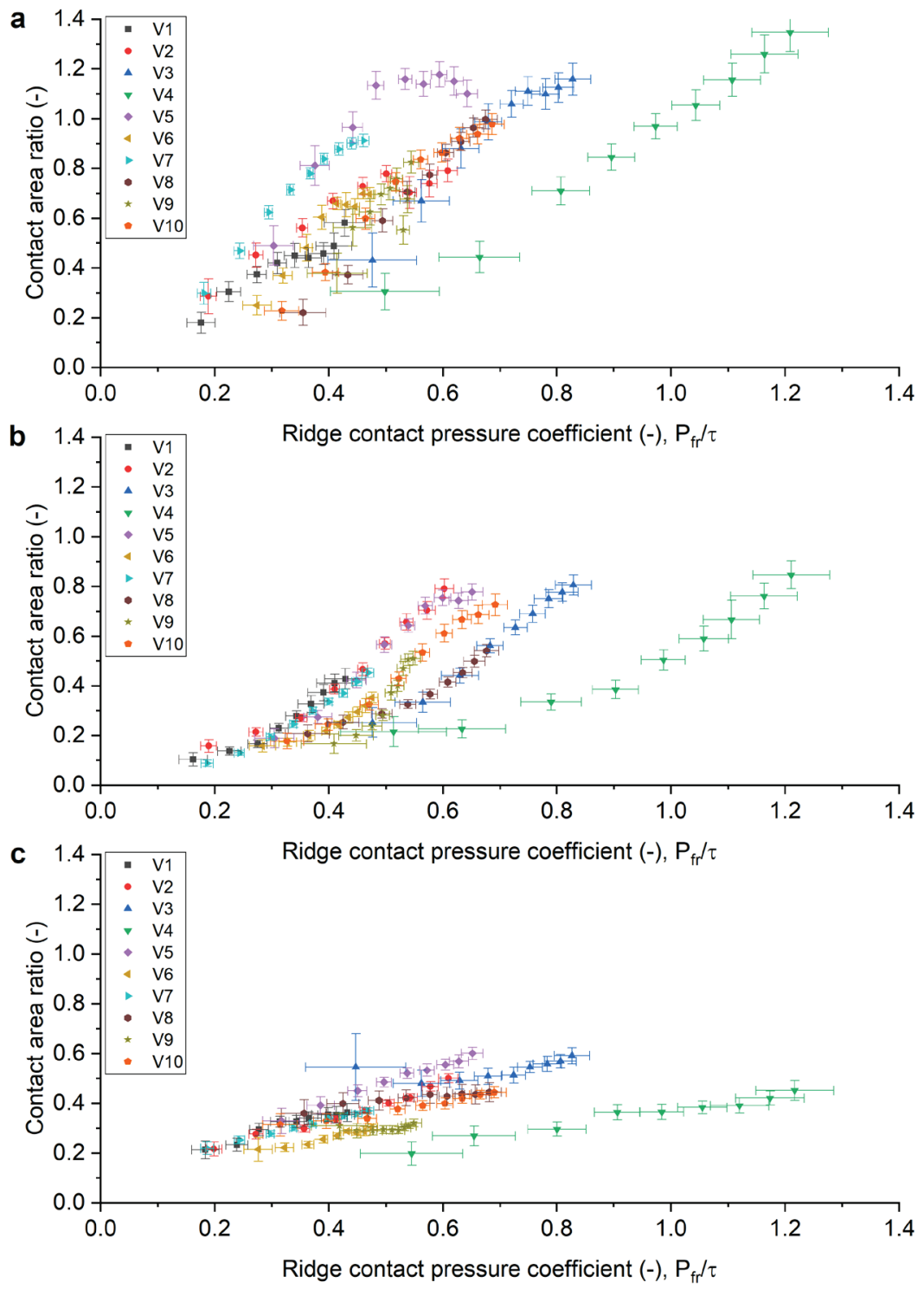

FIGURE 7 Calculated contact area ratio as a function of pressure ratio grouped by texture: S200 (a), E200 (b), S100 (c). 


\section{DISCUSSION}

\subsection{General}

Direct comparison of the absolute friction values between volunteers provides little insight about the origin of the observed differences. Contact conditions for each person differ based on their unique index finger pad structure and skin properties [8]. Even if friction measurements are performed at the same normal load on the macroscale, at the asperity level the ridge contact pressure differs, as it remains inversely proportional to each individual's ridge contact area. Furthermore, interfacial shear strength cannot be considered constant for all volunteers. The values reported in literature vary between $1 \mathrm{kPa}$ and $1.2 \mathrm{MPa}$ for various anatomical locations and materials in contact $[4,28-$ 30].

In this work comparison of the friction results between volunteers is performed by utilising designed deterministic surfaces [24]. The contact with the textured surfaces can be divided in general, into three distinctive stages. At low normal loads, the skin is fully supported by asperities resulting in a reduced real contact area. With increase of the normal load, the skin micro-deformation exceeds the texture height and comes in contact with the lower surface. This increases the real area of contact and ultimately leads to a transition from the asperity contact state to full contact. This transition is characterised by an increase in friction coefficient. Finally, the contact stabilises at full contact state and further rise of the contact area becomes negligible. The effect of contact transition was discussed by other researchers $[7,12,31]$ and an approach to model it was proposed in our previous work [23].

All three contact states are observed for the textured surfaces. Textures S200 and E200 display an increase in friction coefficient as skin deforms and comes in a contact with a texture valley (Figure 4b, c). Elliptic texture E200 has a higher equivalent asperity radius and height, therefore, it can support higher load before contact transition starts. It also showed the lowest coefficient of friction across all subjects at normal loads below $1 \mathrm{~N}$. That confirms the initial asperity contact state, considering that the feature density for E200 texture is lower than that for S100. High density texture S100 remains in asperity contact for all volunteers, which is 
represented in consistently low friction coefficient across all normal loads (Figure 4d). The difference between samples is depicted on Figure 8 for a single volunteer. Interestingly, the texture related trends are independent from the volunteer that tested the samples and can be described in a similar way.

\subsection{Reference value for normalisation}

The validity of using a reference sample for normalisation can be judged by examining the results for the textured surfaces E200 and S100 more closely. E200 showed the lowest coefficient of friction for all participants at normal loads below $1 \mathrm{~N}$. However, at higher loads, friction increased due to the transition to full contact state. The trends for the two textured surfaces E200 and S100 can be compared to estimate a point of intersection, at which friction coefficient and, therefore, contact area ratio become equal for those surfaces. On Figure 8 such intersection can be observed at $1 \mathrm{~N}$ normal load. On the normalised graph this point should correspond to the same contact area ratio value for all participants.

For the linear elastic case, the intersection of those functions corresponds to a specific deformation. Figure 9 shows the matching values for two volunteers, at which friction coefficient and, therefore, contact area ratio becomes equal for those surfaces. Due to the differences in ridge contact area and elastic modulus of the skin, normal load at those points significantly vary between volunteers. Table 2 represents the values for the equivalent point for each participant. The mean contact area fraction for the volunteers averages at 0.37 . However, participants V3, V5 and V8 deviate further from the rest with the values above 0.43 .

The high deviation from other results for these participants indicates a contradiction to the taken assumptions. Therefore, normalisation of their data could be unrepresentative. Interestingly, both volunteers V3 and V8 showed the lowest skin hydration values. Moreover, participants V3 and V5 showed contact area ratio larger than 1 on Figure 7a. This is a probable case if those volunteers had high fingerprint ridge roughness which led to a reduced contact area with a reference sample. 


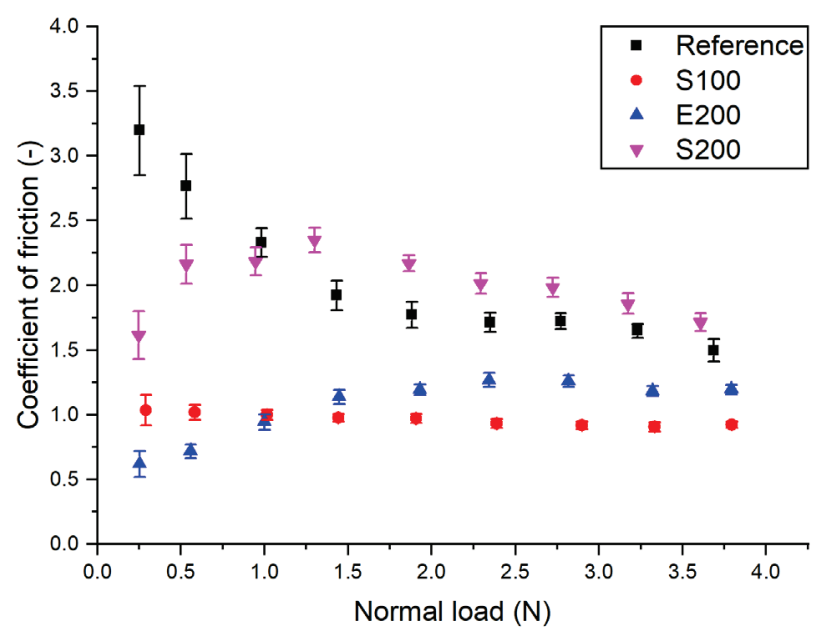

FIGURE 8 Calculated coefficient of friction for the 4 surfaces tested by volunteer V5.

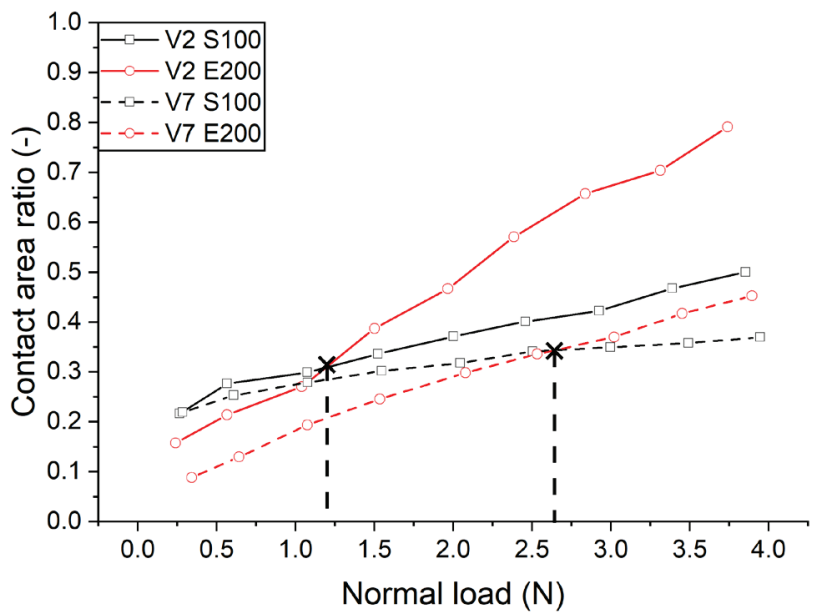

FIGURE 9 Intersection of the calculated contact area ratio trends for textures S100 and E200. 
TABLE 2 Normal loads at which contact area ratio for two surfaces S100 and E200 becomes equal.

\begin{tabular}{|lccccc|} 
& V1 & V2 & V3 & V4 & V5 \\
Contact area ratio, r [-] & 0.35 & 0.31 & 0.50 & 0.35 & 0.46 \\
Normal load, F [N] & 2.57 & 1.19 & 1.27 & 1.27 & 1.12 \\
& & & & & \\
\hline Contact area ratio, r [-] & 0.28 & 0.34 & 0.43 & 0.29 & 0.35 \\
Normal load, F [N] & 2.74 & 2.65 & 2.79 & 1.61 & 1.25
\end{tabular}

\subsection{Normalisation by the point of reference}

Considering that a linear elastic case can be applied for small skin deformations, each dataset is normalised by the intersection point discussed above, which corresponds to a mean contact ratio value of 0.37 . This point of intersection can be clearly defined both for the friction results and a contact model, which allows to apply the same normalisation approach and oppose the assumptions. This operation allows to eliminate the remaining personal differences from the graphs on Figure 7. The convergence of the data for the textured surfaces L200 and S100 means that deformation on the asperity scale remains proportional to pressure for all volunteers. Notably, on Figure 10c volunteers V3, V5 and V8 show higher values than other participants. As discussed above, skin roughness and low skin hydration resulted in reduced friction against reference samples introducing error to the normalised results. 

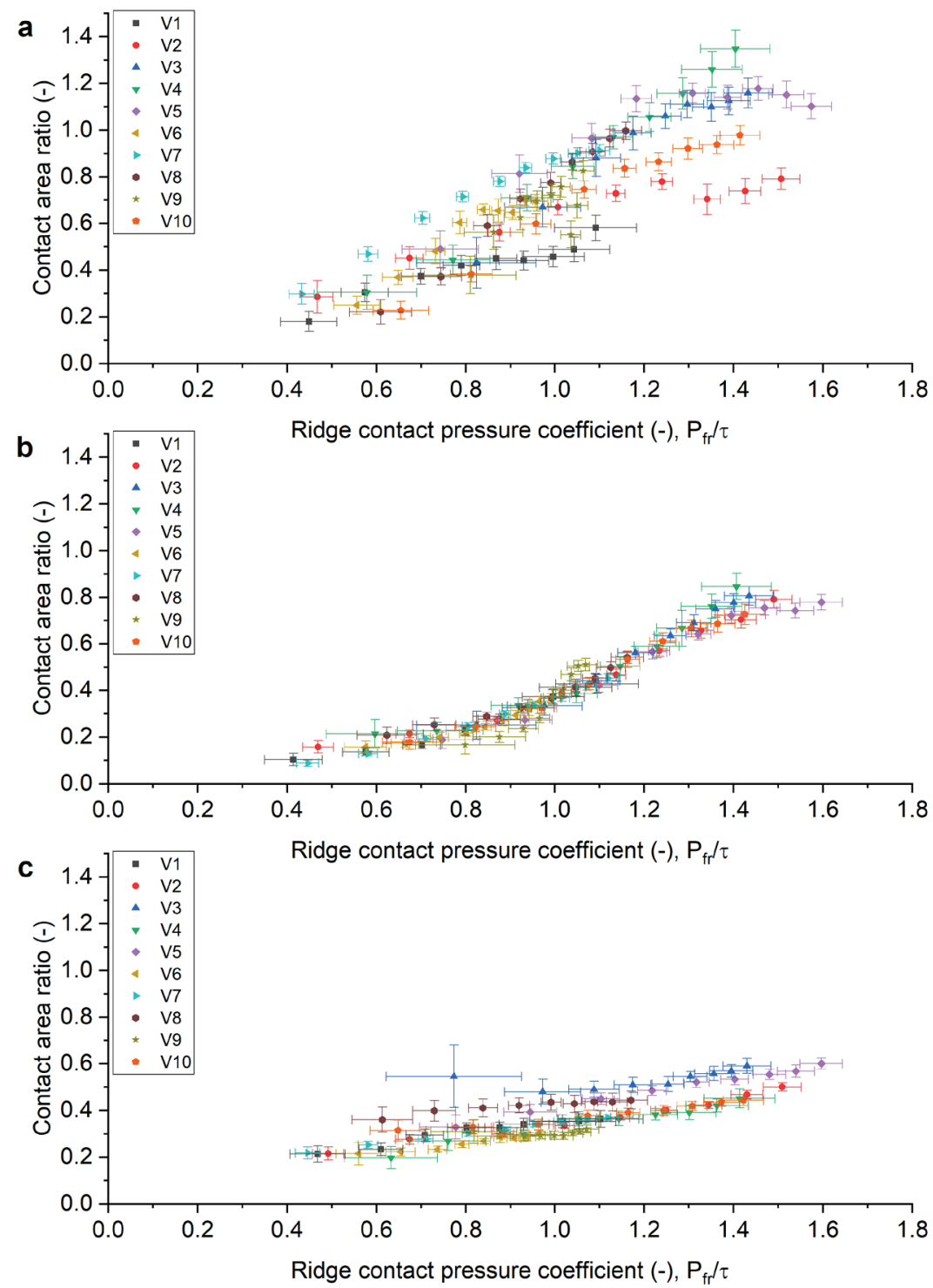

FIGURE 10 Calculated contact area ratio as a function of normalised pressure grouped by texture: S200 (a), E200 (b), S100 (c) 


\subsection{Comparison with numerical model}

A boundary element method (BEM) was used to calculate a contact area ratio for a micro-scale case. The contact was assumed as a half-space linear elastic problem. Skin was assumed ideally flat, while the texture topography was obtained from confocal measurements. Contact area ratio can be plotted against normalised pressure to represent a function of surface topography. In this case effective elastic modulus chosen for the simulation becomes irrelevant. Normally ridge pressure is normalised by the Young's modulus or pressure at maximum contact. To compare the model with experimental results the data is normalised by the point of intersection for two textures E200 and S100.

After the normalisation of pressure, the total deformation range can be compared to experimental results on Figure 11. The modelled results are fit to the frictional data as $r\left(\hat{P}_{f r}\right)=r_{s}\left(\hat{P}_{f r}\right)+0.2 \hat{P}_{f r}$, where $r_{s}\left(\hat{P}_{f r}\right)$ is a contact area ratio function obtained from the numerical model and $\hat{p}_{f r}$ is a mean ridge contact pressure normalised by the reference point. Interestingly, the linear fit does not change with texture or participant. It suggests that coefficient of friction is proportional to pressure. This behaviour is best explained by the interfacial shear strength relation to pressure proposed by Adams et al. [28]. They proposed that in the presence of a thin organic film, such as sebum layer, the interfacial shear strength $\tau$ is not a constant, but a linear function of the real contact pressure $p_{r}$ as follows: $\tau=\tau_{0}+\alpha p_{r}$, where $\tau_{0}$ is the intrinsic interfacial shear strength and $\alpha$ is a pressure coefficient. For textured surfaces average real contact pressure is expected to be several times higher, which leads to higher interfacial shear strength for the same normal load.

Interestingly, the volunteer data lies within a small range of normalised ridge pressure values. The participants experienced a similar range of skin micro-deformations, even though the personal differences were prominent. For this to hold true, the higher skin elastic modulus must be accompanied by a corresponding increase in ridge pressure and vice versa, which can be achieved by the deformation of fingerprint ridges. This could explain why perception of surface textures is closely related to texture dimensions and similar textures receive uniform ratings [32,33]. 


\subsection{Assumptions and limitations}

The validity of the assumptions taken in section 3 can be discussed based on the observed datasets. The first assumption states that ridge contact area does not change with the sample. Considering that the sample order was randomised, and hands were washed between sample measurements, only minor deviations can be observed. Change in the ridge contact area function was presumably observed for participant V9. It represented in a deviation of corneometer readings prior to the test with E200 and resulted in a noticeable divergence from the general trend (Figure $7 \mathrm{~b}$ ). For most of the volunteers the skin hydration level remained stable during the measurement procedure. However, one should exercise caution if the experiments are performed across several days.

The second assumption states that real contact area for a reference sample approaches ridge contact area. It can be considered viable if skin and surface roughness is negligible or at least one of the surfaces is compliant. In the current study the combination of silicone rubber material with a relatively low surface average roughness allows to satisfy the assumption. Power law fit exponents are close to the theoretical exponent of $2 / 3$, predicted for adhesive contact and experimental values from other studies $[6,13,28,34]$. With increase of the reduced Young's modulus or surface roughness, real contact area reduces leading to errors during normalisation procedure as it was observed with the participants V3, V5 and V8.
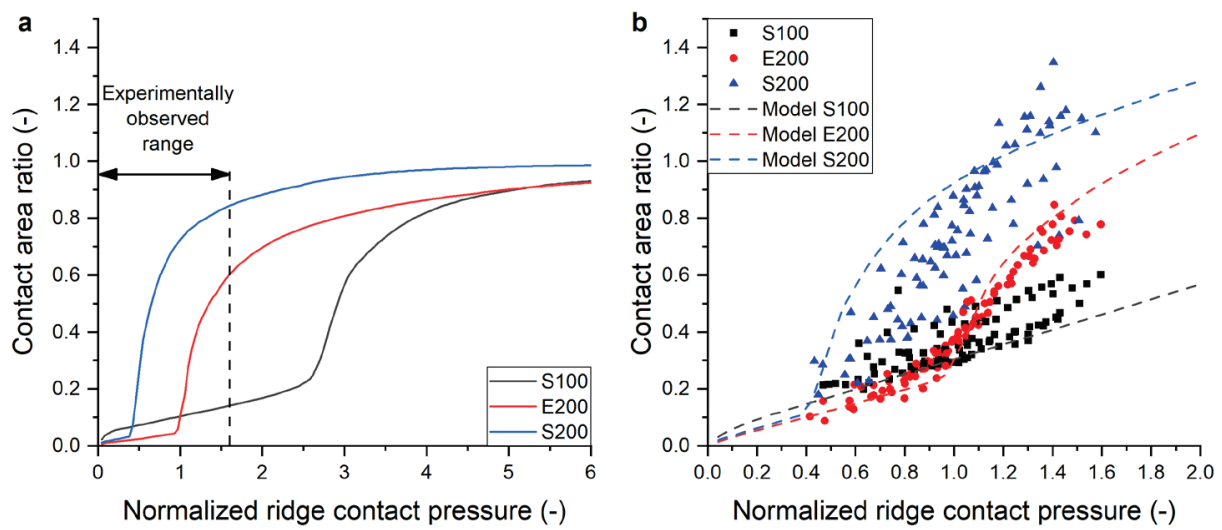

FIGURE 11 Contact model results for textured surfaces (a) and its fit to the normalised friction data (b). 
The third assumption considers only adhesion friction. For the dry finger pad sliding against smooth surfaces, adhesion is considered to be a predominant component of friction [16-18]. Tomlinson et al. [35] reported a $10 \%$ deformation term for the triangular ridged surfaces with the heights over $250 \mu \mathrm{m}$. Moreover, they found an interlocking term to become considerable for surfaces with heights above $40 \mu \mathrm{m}$. Textures used in this study were designed to minimise interlocking and have smaller feature sizes. However, the interfacial shear strength seems to be dependent on pressure as discussed by Adams et al. [28]. It leads to overestimation of contact area fraction after normalisation, which increases linearly with pressure and does not change with participant.

\section{CONCLUSIONS}

- Normalisation of the frictional data allows to reduce one of the unknown personal variables: function of the ridge contact area, reduced Young's modulus or interfacial shear strength. Furthermore, the results can be normalised to dimensionless values to compare the contact area development on the microscale. The resulted graphs confirm interfacial shear strength relation to contact pressure.

- All subjects experienced the same contact states with the tested textures, despite the personal differences, contact area and skin hydration levels.

- This suggests that deformation of the skin on the microscale is independent of the skin elasticity due to the fingerprint ridge pattern. In other words, the change of the skin Young's modulus is closely related to the reduction of the ridge contact area and, therefore, increase of the ridge pressure, leading to comparable displacement values.

\section{Ethical approval}

The procedures performed in the current study were approved by the Natural Sciences and Engineering Sciences Ethics committee, University of Twente, Enschede. The measurements were carried out in compliance with COVID19 government regulations active during that time. 
All subjects provided a written informed consent. None of the experiments were invasive or harmful physiologically or psychologically. All data was stored anonymously, and the participants had the right to quit at any time if they so wished.

\section{Acknowledgements}

This work was supported by INTERREG V-A Deutschland - Nederland program MOVERO under the project number 142091.

\section{REFERENCES}

[1] G.D. Lamb, Tactile discrimination of textured surfaces: psychophysical performance measurements in humans, J. Physiol. 338 (1983) 551-565. doi:10.1113/jphysiol.1983.sp014689.

[2] N.K. Veijgen, E. van der Heide, M.A. Masen, A multivariable model for predicting the frictional behaviour and hydration of the human skin, Ski. Res. Technol. 19 (2013) 330-338. doi:10.1111/srt.12053.

[3] X. Liu, Z. Lu, R. Lewis, M.J. Carré, S.J. Matcher, Feasibility of using optical coherence tomography to study the influence of skin structure on finger friction, Tribol. Int. 63 (2013) 34-44. doi:10.1016/j.triboint.2012.08.020.

[4] C.P. Hendriks, S.E. Franklin, Influence of surface roughness, material and climate conditions on the friction of human skin, Tribol. Lett. 37 (2010) 361-373. doi:10.1007/s11249-009-9530-7.

[5] K.L. Johnson, Contact Mechanics, Cambridge University Press, Cambridge, 1985.

[6] X. Liu, M.J. Carré, Q. Zhang, Z. Lu, S.J. Matcher, R. Lewis, Measuring contact area in a sliding human finger-pad contact, Ski. Res. Technol. 24 (2018) 3144. doi:10.1111/srt.12387.

[7] J. Van Kuilenburg, M.A. Masen, E. van der Heide, The role of the skin microrelief in the contact behaviour of human skin: Contact between the human finger and regular surface textures, Tribol. Int. 65 (2013) 81-90. doi:10.1016/j.triboint.2012.11.024.

[8] B.M. Dzidek, M.J. Adams, J.W. Andrews, Z. Zhang, S.A. Johnson, Contact mechanics of the human finger pad under compressive loads., J. R. Soc. Interface. 14 (2017) 20160935. doi:10.1098/rsif.2016.0935.

[9] T. Soneda, K. Nakano, Investigation of vibrotactile sensation of human fingerpads by observation of contact zones, Tribol. Int. 43 (2010) 210-217. doi:10.1016/j.triboint.2009.05.016.

[10] M.J. Adams, S.A. Johnson, P. Lefèvre, V. Lévesque, V. Hayward, T. André, J.L.L. Thonnard, Finger pad friction and its role in grip and touch, J. R. Soc. Interface. 10 (2013) 20120467. doi:10.1098/rsif.2012.0467. 
[11] R. Fagiani, M. Barbieri, Modelling of finger-surface contact dynamics, Tribol. Int. 74 (2014) 130-137. doi:10.1016/j.triboint.2014.02.018.

[12] A.C. Rodríguez Urribarrí, E. van der Heide, X. Zeng, M.B. de Rooij, Modelling the static contact between a fingertip and a rigid wavy surface, Tribol. Int. 102 (2016) 114-124. doi:10.1016/j.triboint.2016.05.028.

[13] T.H.C. Childs, B. Henson, Human tactile perception of screen-printed surfaces: Self-report and contact mechanics experiments, Proc. Inst. Mech. Eng. Part J J. Eng. Tribol. 221 (2007) 427-441. doi:10.1243/13506501JET217.

[14] R. Sahli, G. Pallares, C. Ducottet, I.E. Ben Ali, S. Al Akhrass, M. Guibert, J. Scheibert, Evolution of real contact area under shear and the value of static friction of soft materials, Proc. Natl. Acad. Sci. 115 (2018) 471-476. doi:10.1073/pnas.1706434115.

[15] H.T. Lin, T.F. Hong, W.L. Li, Grip performance affected by water-induced wrinkling of fingers, Tribol. Lett. 58 (2015) 1-9. doi:10.1007/s11249-0150515-4.

[16] S. Derler, L.C. Gerhardt, A. Lenz, E. Bertaux, M. Hadad, Friction of human skin against smooth and rough glass as a function of the contact pressure, Tribol. Int. 42 (2009) 1565-1574. doi:10.1016/j.triboint.2008.11.009.

[17] K. Duvefelt, U. Olofsson, C.M. Johannesson, L. Skedung, Model for contact between finger and sinusoidal plane to evaluate adhesion and deformation component of friction, Tribol. Int. 96 (2016) 389-394. doi:10.1016/j.triboint.2014.12.020.

[18] S. Derler, G.M. Rotaru, Stick-slip phenomena in the friction of human skin, Wear. 301 (2013) 324-329. doi:10.1016/j.wear.2012.11.030.

[19] M. Klaassen, D.J. Schipper, M.A. Masen, Influence of the relative humidity and the temperature on the in-vivo friction behaviour of human skin, Biotribology. 6 (2016) 21-28. doi:10.1016/j.biotri.2016.03.003.

[20] N.K. Veijgen, M.A. Masen, E. van der Heide, Variables influencing the frictional behaviour of in vivo human skin, J. Mech. Behav. Biomed. Mater. 28 (2013) 448-461. doi:10.1016/j.jmbbm.2013.02.009.

[21] N.K. Veijgen, M.A. Masen, E. Van Der Heide, Relating friction on the human skin to the hydration and temperature of the skin, Tribol. Lett. 49 (2013) 251-262. doi:10.1007/s11249-012-0062-1.

[22] M. Arvidsson, L. Ringstad, L. Skedung, K. Duvefelt, M.W. Rutland, Feeling fine - the effect of topography and friction on perceived roughness and slipperiness, Biotribology. $11 \quad$ (2017) 92-101. doi:10.1016/j.biotri.2017.01.002.

[23] D.A. Sergachev, D.T.A. Matthews, E. van der Heide, An Empirical Approach for the Determination of Skin Elasticity: Finger pad Friction against $\begin{array}{lllll}\text { Textured } & \text { Surfaces, } & \text { Biotribology. } & 18 & \text { (2019) }\end{array}$ doi:10.1016/j.biotri.2019.100097.

[24] D.A. Sergachev, D.T.A. Matthews, E. van der Heide, Design of bidirectional frictional behaviour for tactile contact using ellipsoidal asperity micro- 
textures, (2021).

[25] R.L. Jackson, I. Green, On the modeling of elastic contact between rough surfaces, Tribol. Trans. 54 (2011) 300-314. doi:10.1080/10402004.2010.542277.

[26] V.A. Yastrebov, G. Anciaux, J.F. Molinari, From infinitesimal to full contact between rough surfaces: Evolution of the contact area, Int. J. Solids Struct. 52 (2015) 83-102. doi:10.1016/j.ijsolstr.2014.09.019.

[27] U. Heinrich, U. Koop, M.C. Leneveu-Duchemin, K. Osterrieder, S. Bielfeldt, C. Chkarnat, J. Degwert, D. Häntschel, S. Jaspers, H.P. Nissen, M. Rohr, G. Schneider, H. Tronnier, Multicentre comparison of skin hydration in terms of physical-, physiological- and product-dependent parameters by the capacitive method (Corneometer CM 825), Int. J. Cosmet. Sci. 25 (2003) 4553. doi:10.1046/j.1467-2494.2003.00172.x.

[28] M.J. Adams, B.J. Briscoe, S.A. Johnson, Friction and lubrication of human skin, Tribol. Lett. 26 (2007) 239-253. doi:10.1007/s11249-007-9206-0.

[29] S. Bochereau, B. Dzidek, M. Adams, V. Hayward, Characterizing and imaging gross and real finger contacts under dynamic loading, IEEE Trans. Haptics. 10 (2017) 456-466. doi:10.1109/TOH.2017.2686849.

[30] S. Derler, G.-M.M. Rotaru, W. Ke, L. El Issawi-Frischknecht, P. Kellenberger, A. Scheel-Sailer, R.M.M. Rossi, Microscopic contact area and friction between medical textiles and skin, J. Mech. Behav. Biomed. Mater. 38 (2014) 114-125. doi:10.1016/j.jmbbm.2014.06.014.

[31] S. Zhang, A. Rodriguez Urribarri, M. Morales Hurtado, X. Zeng, E. van der Heide, The role of the sliding direction against a grooved channel texture on tool steel: An experimental study on tactile friction, Int. J. Solids Struct. 56 (2015) 53-61. doi:10.1016/j.ijsolstr.2014.12.005.

[32] V. Massimiani, B. Weiland, E. Chatelet, P.H. Cornuault, J. Faucheu, F. Massi, The role of mechanical stimuli on hedonistic and topographical discrimination of textures, Tribol. Int. $143 \quad$ (2020) 106082. doi:10.1016/j.triboint.2019.106082.

[33] J. Faucheu, B. Weiland, M. Juganaru-Mathieu, A. Witt, P.H. Cornuault, Tactile aesthetics: Textures that we like or hate to touch, Acta Psychol. (Amst). 201 (2019) 102950. doi:10.1016/j.actpsy.2019.102950.

[34] M.A. Darden, C.J. Schwartz, Investigation of friction mechanisms during the sliding of elastomers against hard parallel-ridge textures, Tribol. Int. 63 (2013) 2-7. doi:10.1016/j.triboint.2012.01.005.

[35] S.E. Tomlinson, M.J. Carré, R. Lewis, S.E. Franklin, Human finger contact with small, triangular ridged surfaces, Wear. 271 (2011) 2346-2353. doi:10.1016/j.wear.2010.12.055. 



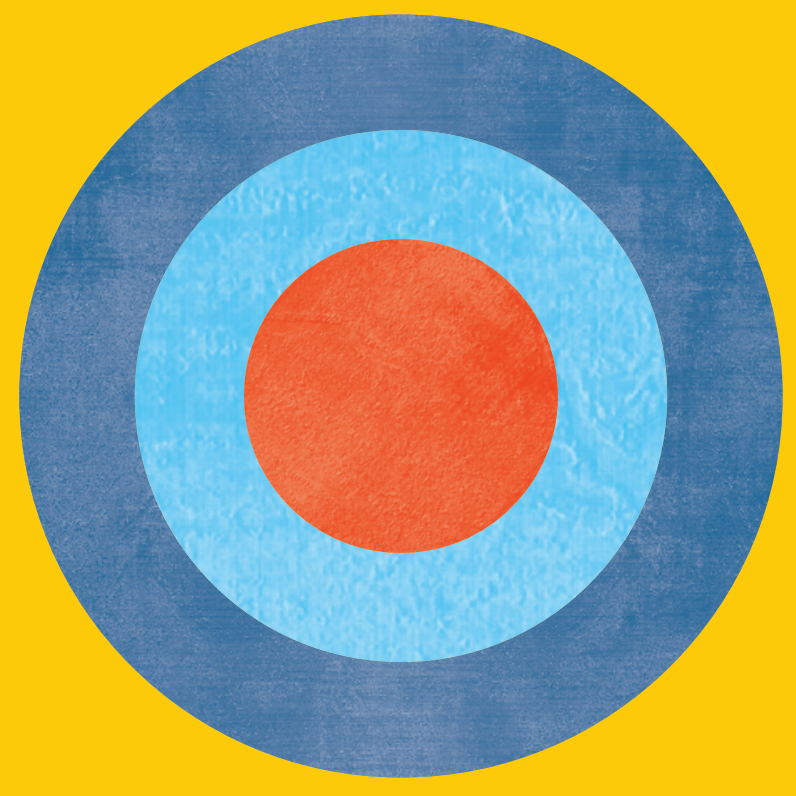

